\title{
Catalytic Asymmetric Rearrangement of $\alpha, \alpha$-Dialkyl- $\alpha$-siloxy Aldehydes to Optically Active Acyloins Using Axially Chiral Organoaluminum Lewis Acids
}

\author{
Takashi Ooi, Kohsuke Ohmatsu and Keiji Maruoka* \\ Department of Chemistry, Graduate School of Science, Kyoto University \\ Sakyo, Kyoto 606-8502, Japan
}

\section{Supporting Information}

I. General. Infrared (IR) Spectra were recorded on a Shimadzu FT-IR. ${ }^{1} \mathrm{H}$ and ${ }^{13} \mathrm{C}$ NMR spectra were measured on JEOL JNM-FX400 (400 MHz) and JEOL AL-400 (400 $\mathrm{MHz}$ ) spectrometers. Chemical shifts of ${ }^{1} \mathrm{H}$ NMR spectra were reported in ppm relative to tetramethylsilane $(\delta 0)$. Chemical shifts of ${ }^{13} \mathrm{C}$ NMR spectra were reported in ppm relative to the residual solvent (chloroform, $\delta$ 77.07). Analytical high performance liquid chromatography (HPLC) was performed on a Shimadzu LC-10AT instrument equipped with a column of Daicel Chiralcel OD-H, OJ-H, Chiralpak AD-H or AS-H. Optical rotations were measured on a JASCO DIP-1000 digital polarimeter. The highresolution mass spectra (HRMS) were measured on an BRUKER DALTONICS microTOF focus-KR spectrometer. Analytical thin layer chromatography (TLC) was performed on Merck precoated TLC plates (silica gel $60 \mathrm{GF}_{254}, 0.25 \mathrm{~mm}$ ) throughout this work. Flash column chromatography was performed on silica gel 60 (Merck 1.09386.9025, 230-400 mesh). An EYELA PSL-1400 constant temperature bath was used for low temperature asymmetric rearrangement reactions.

All air- and moisture-sensitive reactions were performed under an atmosphere of argon in flame-dried, round bottom flasks fitted with rubber septa. The manipulations for asymmetric rearrangements were carried out with standard Schlenk techniques under nitrogen. In experiments requiring dry solvent, toluene was purified by both A2 alumina and Q5 reactant using a GlassContour solvent dispensing system. Ethylene glycol dimethyl ether (DME) was dried over $4 \AA$ molecular sieves pellets. Diethyl ether and Tetrahydrofuran (THF) were purchased from Kanto Chemical Co., Ltd. as “dehydrated". Trimethylaluminum was kindly supplied from Tosoh-Finechem Co., Ltd., Japan. Other simple chemicals were purchased and used as such. 


\section{Experimental Procedure:}

\section{Preparation of Substrates}

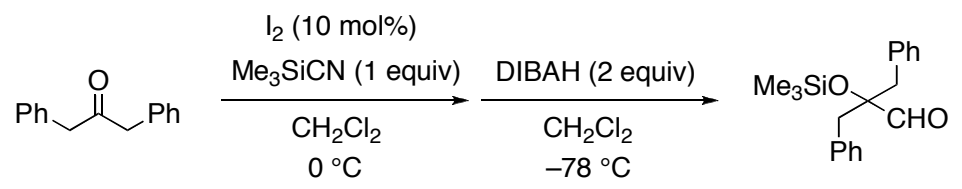

\section{2-Benzyl-3-phenyl-2-trimethylsiloxypropanal $\left[1 \mathrm{a}\left(\mathrm{R}^{2}=\mathrm{Me}\right)\right]$}

To a solution of 1,3-diphenyl-2-propanone $(2.10 \mathrm{~g}, 10 \mathrm{mmol})$ and trimethylsilyl cyanide $(1.33 \mathrm{~mL}, 10 \mathrm{mmol})$ in $\mathrm{CH}_{2} \mathrm{Cl}_{2}(30 \mathrm{~mL})$ was added $\mathrm{I}_{2}(253 \mathrm{mg}, 1 \mathrm{mmol})$ at $0{ }^{\circ} \mathrm{C}$ under argon. After stirring for $30 \mathrm{~min}$ at $0{ }^{\circ} \mathrm{C}$, the reaction was quenched with saturated $\mathrm{Na}_{2} \mathrm{SO}_{3}$ and extractive workup was conducted with ether. The organic extracts were then washed with brine and dried over anhydrous $\mathrm{Na}_{2} \mathrm{SO}_{4}$. After evaporation of solvents and drying under vacuum, the crude products were dissolved into $\mathrm{CH}_{2} \mathrm{Cl}_{2}(20 \mathrm{~mL})$ under argon. A $1 \mathrm{M}$ toluene solution of DIBAH $(20 \mathrm{~mL}, 20 \mathrm{mmol})$ was introduced to this solution at $-78{ }^{\circ} \mathrm{C}$, and the reaction mixture was stirred for $1 \mathrm{~h}$ at the same temperature. Then, the mixture was quenched with $1 \mathrm{~N} \mathrm{HCl}$ and extracted with ether. The organic extracts were washed with brine and dried over anhydrous $\mathrm{Na}_{2} \mathrm{SO}_{4}$, and concentrated. The residual oil was purified by column chromatography on silica gel $\left(\mathrm{CH}_{2} \mathrm{Cl}_{2} /\right.$ hexane $=$ $1 / 4$ as eluant) to give the title compound (2.97 $\mathrm{g}, 9.5 \mathrm{mmol}, 95 \%$ yield) as white solid. ${ }^{1} \mathrm{H}$ NMR (400 MHz, $\left.\mathrm{CDCl}_{3}\right) \delta 9.66$ (1H, s, CHO), 7.21-7.28 (6H, m, Ph-H), 7.13-7.15 (4H, m, Ph-H), 3.07 (2H, d, $\left.J=14.0 \mathrm{~Hz}, \mathrm{PhCH}_{2}\right), 2.87\left(2 \mathrm{H}, \mathrm{d}, J=14.0 \mathrm{~Hz}, \mathrm{PhCH}_{2}\right)$, $-0.16\left(9 \mathrm{H}, \mathrm{s}, \mathrm{SiMe}_{3}\right) ;{ }^{13} \mathrm{C} \mathrm{NMR}\left(100 \mathrm{MHz}, \mathrm{CDCl}_{3}\right) \delta$ 202.8, 135.5, 130.6, 127.9, 126.7, 84.8, 43.8, 2.2; IR (neat) 3030, 2953, 1741, 1494, 1454, 1247, 1143, 985, 837, 750, 684, $630 \mathrm{~cm}^{-1}$; HRMS (ESI-TOF) Calcd. for $\mathrm{C}_{19} \mathrm{H}_{24} \mathrm{NaO}_{2} \mathrm{Si}\left([\mathrm{M}+\mathrm{Na}]^{+}\right)$: 335.1438, Found: 335.1450. Other substrates were synthesized by the same procedure from known ketones $^{1}$ and cyanosilanes.

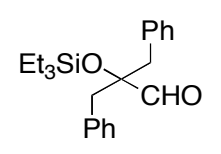

\section{2-Benzyl-3-phenyl-2-triethylsiloxypropanal [1a $\left.\left(\mathrm{R}^{2}=\mathrm{Et}\right)\right]$}

${ }^{1} \mathrm{H}$ NMR (400 MHz, $\mathrm{CDCl}_{3}$ ) $\delta 9.64(1 \mathrm{H}, \mathrm{s}, \mathrm{CHO}), 7.21-7.27$ (6H, m, PhH), 7.14-7.16 (4H, m, Ph-H), 3.06 (2H, d, $\left.J=14.0 \mathrm{~Hz}, \mathrm{PhCH}_{2}\right), 2.89$ (2H, $\left.\mathrm{d}, J=14.0 \mathrm{~Hz}, \mathrm{PhCH}_{2}\right), 0.82\left(9 \mathrm{H}, \mathrm{t}, J=8.0 \mathrm{~Hz}, \mathrm{CH}_{3} \mathrm{CH}_{2} \mathrm{Si}\right), 0.45(6 \mathrm{H}, \mathrm{q}, J=8.0 \mathrm{~Hz}$, $\left.\mathrm{CH}_{3} \mathrm{C}_{2} \mathrm{Si}\right) ;{ }^{13} \mathrm{C} \mathrm{NMR}\left(100 \mathrm{MHz}, \mathrm{CDCl}_{3}\right) \delta 203.2,135.4,130.6,127.9,126.5,83.7,43.6$, 7.2, 6.9; IR (neat) 2953, 2910, 2873, 2362, 1739, 1494, 1454, 1238, 1141, 1091, 1002, 729, 698, $669 \mathrm{~cm}^{-1}$; HRMS (ESI-TOF) Calcd. for $\mathrm{C}_{22} \mathrm{H}_{31} \mathrm{O}_{2} \mathrm{Si}\left([\mathrm{M}+\mathrm{H}]^{+}\right): 355.2088$, Found: 355.2087. 


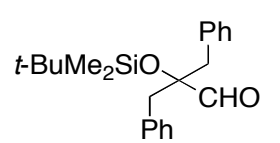

2-Benzyl-2-tert-butyldimethylsiloxy-3-phenylpropanal $\left[1 \mathrm{a}\left(\mathrm{R}_{3}^{2}=t\right.\right.$ $\left.\left.\mathrm{BuMe}_{2}\right)\right]$

${ }^{1} \mathrm{H}$ NMR (400 MHz, $\left.\mathrm{CDCl}_{3}\right) \delta 9.67(1 \mathrm{H}, \mathrm{s}, \mathrm{CHO}), 7.21-7.27$ (6H, m, Ph-H), 7.14-7.16 (4H, m, Ph-H), $3.12\left(2 \mathrm{H}, \mathrm{d}, J=14.0 \mathrm{~Hz}, \mathrm{PhCH}_{2}\right), 2.94(2 \mathrm{H}, \mathrm{d}, J=$ $\left.14.0 \mathrm{~Hz}, \mathrm{PhCH}_{2}\right), 0.87(9 \mathrm{H}, \mathrm{s}, t-\mathrm{BuSi}),-0.24\left(6 \mathrm{H}, \mathrm{s}, \mathrm{Me}_{2} \mathrm{Si}\right) ;{ }^{13} \mathrm{C}$ NMR $(100 \mathrm{MHz}$, $\mathrm{CDCl}_{3}$ ) \& 203.4, 135.5, 130.8, 128.1, 126.8, 84.3, 44.5, 26.3, 18.9, -2.7; IR (neat) 3030, 2953, 2927, 2856, 2358, 1722, 1496, 1471, 1454, 1253, 1093, 943, 835, 812, 777, 750, 732, 698, $669 \mathrm{~cm}^{-1}$; HRMS (ESI-TOF) Calcd. for $\mathrm{C}_{22} \mathrm{H}_{30} \mathrm{NaO}_{2} \mathrm{Si}\left([\mathrm{M}+\mathrm{Na}]^{+}\right)$: 377.1918 , Found: 377.1920 .

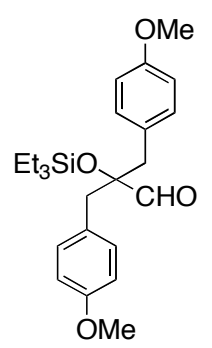

2-(4-Methoxybenzyl)-3-(4-methoxyphenyl)-2-triethylsiloxypropanal (1b)

${ }^{1} \mathrm{H}$ NMR $\left(400 \mathrm{MHz}, \mathrm{CDCl}_{3}\right) \delta 9.60(1 \mathrm{H}, \mathrm{s}, \mathrm{CHO}), 7.06(4 \mathrm{H}, \mathrm{d}, J=6.8 \mathrm{~Hz}$, Ar-H), 6.79 (4H, d, $J=6.8 \mathrm{~Hz}, \mathrm{Ar}-\mathrm{H}), 3.78$ (6H, s, MeOPh), $2.93(2 \mathrm{H}, \mathrm{d}$, $\left.J=14.0 \mathrm{~Hz}, \mathrm{PhCH}_{2}\right), 2.82\left(2 \mathrm{H}, \mathrm{d}, J=14.0 \mathrm{~Hz}, \mathrm{PhCH}_{2}\right), 0.85(9 \mathrm{H}, \mathrm{t}, J=$ $\left.8.0 \mathrm{~Hz}, \mathrm{CH}_{3} \mathrm{CH}_{2} \mathrm{Si}\right), 0.48\left(6 \mathrm{H}, \mathrm{q}, J=8.0 \mathrm{~Hz}, \mathrm{CH}_{3} \mathrm{CH}_{2} \mathrm{Si}\right) ;{ }^{13} \mathrm{C}$ NMR $(100$ $\left.\mathrm{MHz}, \mathrm{CDCl}_{3}\right) \delta$ 203.6, 158.2, 131.4, 127.4, 113.3, 83.8, 55.1, 42.5, 7.2, 6.9; IR (neat) 2953, 2910, 2873, 1739, 1612, 1512, 1463, 1301, 1247, 1176, 1143, 1035, 829, $732 \mathrm{~cm}$ '; HRMS (ESI-TOF) Calcd. for $\mathrm{C}_{24} \mathrm{H}_{34} \mathrm{NaO}_{4} \mathrm{Si}\left([\mathrm{M}+\mathrm{Na}]^{+}\right)$: 437.2119, Found: 437.2132.

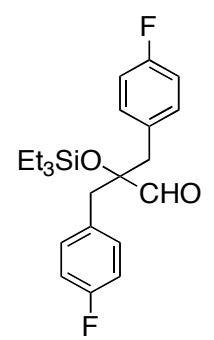

\section{2-(4-Fluorobenzyl)-3-(4-fluorophenyl)-2-triethylsiloxypropanal (1c)}

${ }^{1} \mathrm{H}$ NMR (400 MHz, $\left.\mathrm{CDCl}_{3}\right) \delta 9.61(1 \mathrm{H}, \mathrm{s}, \mathrm{CHO}), 7.08-7.25$ (4H, m, ArH), 6.92-6.97 (4H, m, Ar-H), $2.94\left(2 \mathrm{H}, \mathrm{d}, J=14.0 \mathrm{~Hz}, \mathrm{PhCH}_{2}\right), 2.83(2 \mathrm{H}$, d, $\left.J=14.0 \mathrm{~Hz}, \mathrm{PhCH}_{2}\right), 0.84\left(9 \mathrm{H}, \mathrm{t}, J=8.0 \mathrm{~Hz}, \mathrm{CH}_{3} \mathrm{CH}_{2} \mathrm{Si}\right), 0.48(6 \mathrm{H}, \mathrm{q}, J$ $\left.=8.0 \mathrm{~Hz}, \mathrm{CH}_{3} \mathrm{C}_{2} \mathrm{Si}\right) ;{ }^{13} \mathrm{C} \mathrm{NMR}\left(100 \mathrm{MHz}, \mathrm{CDCl}_{3}\right) \delta 203.0,161.7\left(\mathrm{~d}, J_{\mathrm{C}-\mathrm{F}}\right.$ $=246 \mathrm{~Hz}), 132.0\left(\mathrm{~d}, J_{\mathrm{C}-\mathrm{F}}=7.4 \mathrm{~Hz}\right), 131.0\left(\mathrm{~d}, J_{\mathrm{C}-\mathrm{F}}=3.3 \mathrm{~Hz}\right), 114.7\left(\mathrm{~d}, J_{\mathrm{C}-\mathrm{F}}=20.6 \mathrm{~Hz}\right)$, 83.6, 42.4, 7.1, 6.9; IR (neat) 2953, 2910, 2873, 1741, 1585, 1490, 1456, 1413, 1230, 1184, 1147, 1114, 1002, 983, 937, 840, 754, 727, $671 \mathrm{~cm}^{-1}$; HRMS (ESI-TOF) Calcd. for $\mathrm{C}_{22} \mathrm{H}_{28} \mathrm{~F}_{2} \mathrm{NaO}_{2} \mathrm{Si}\left([\mathrm{M}+\mathrm{Na}]^{+}\right)$: 413.1730, Found: 413.1738 .

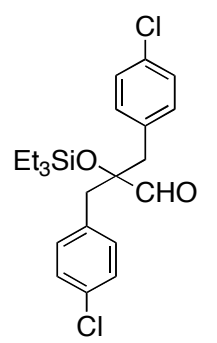

\section{2-(4-Chlorobenzyl)-3-(4-chlorophenyl)-2-triethylsiloxypropanal (1d)}

${ }^{1} \mathrm{H}$ NMR $\left(400 \mathrm{MHz}, \mathrm{CDCl}_{3}\right) \delta 9.60(1 \mathrm{H}, \mathrm{s}, \mathrm{CHO}), 7.23(4 \mathrm{H}, \mathrm{d}, J=8.0 \mathrm{~Hz}$ Ar-H), 7.07 (4H, d, $J=8.0 \mathrm{~Hz}, \mathrm{Ar}-\mathrm{H}), 2.93\left(2 \mathrm{H}, \mathrm{d}, J=14.0 \mathrm{~Hz}, \mathrm{PhCH}_{2}\right)$, $2.83\left(2 \mathrm{H}, \mathrm{d}, J=14.0 \mathrm{~Hz}, \mathrm{PhCH}_{2}\right), 0.85\left(9 \mathrm{H}, \mathrm{t}, J=8.0 \mathrm{~Hz}, \mathrm{CH}_{3} \mathrm{CH}_{2} \mathrm{Si}\right)$, $0.48\left(6 \mathrm{H}, \mathrm{q}, J=8.0 \mathrm{~Hz}, \mathrm{CH}_{3} \mathrm{CH}_{2} \mathrm{Si}\right) ;{ }^{13} \mathrm{C} \mathrm{NMR}\left(100 \mathrm{MHz}, \mathrm{CDCl}_{3}\right) \delta 203.1$, 133.8, 132.8, 132.0, 128.2, 83.5, 42.6, 7.0, 6.8; IR (neat) 2954, 2910, 2875, 1741, 1490, 1409, 1240, 1143, 1089, 1016, 987, 825, 806, $727 \mathrm{~cm}^{-1}$; HRMS (ESI-TOF) Calcd. for $\mathrm{C}_{22} \mathrm{H}_{28} \mathrm{Cl}_{2} \mathrm{NaO}_{2} \mathrm{Si}\left([\mathrm{M}+\mathrm{Na}]^{+}\right): 445.1139$, Found: 445.1138 . 


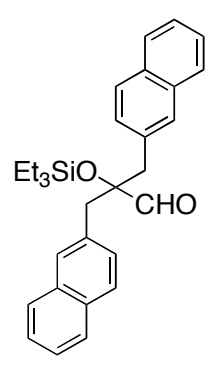

2-(2-Naphthylmethyl)-3-(2-naphthyl)-2-triethylsiloxypropanal (1e)

${ }^{1} \mathrm{H}$ NMR (400 MHz, $\left.\mathrm{CDCl}_{3}\right) \delta 9.71(1 \mathrm{H}, \mathrm{s}, \mathrm{CHO}), 7.74-7.82(6 \mathrm{H}, \mathrm{m}, \mathrm{Ar}-$ H), 7.63 (2H, s, Ar-H), 7.42-7.47 (4H, m, Ph-H), 7.25-7.32 (2H, m, Ar-H), $3.19\left(2 \mathrm{H}, \mathrm{d}, J=14.0 \mathrm{~Hz}, \mathrm{PhCH}_{2}\right), 3.11\left(2 \mathrm{H}, \mathrm{d}, J=14.0 \mathrm{~Hz}, \mathrm{PhCH}_{2}\right), 0.82$ $\left(9 \mathrm{H}, \mathrm{t}, J=8.0 \mathrm{~Hz}, \mathrm{C}_{3} \mathrm{CH}_{2} \mathrm{Si}\right), 0.50\left(6 \mathrm{H}, \mathrm{q}, J=8.0 \mathrm{~Hz}, \mathrm{CH}_{3} \underline{\mathrm{C}}_{2} \mathrm{Si}\right) ;{ }^{13} \mathrm{C}$ NMR $\left(100 \mathrm{MHz}, \mathrm{CDCl}_{3}\right) \delta 203.2,133.1,133.0,132.2,129.3,128.8$, 127.4, 127.3, 127.3, 125.8, 125.4, 83.9, 43.6, 7.2, 7.0; IR (neat) 3055, 2953, 2908, 2873, 2357, 2322, 1732, 1600, 1508, 1456, 1379, 1367, 1271, 1240, 1157, 1136, 1124, 1001, 956, 891, 854, 815, 732, $669 \mathrm{~cm}^{-1}$; HRMS (ESI-TOF) Calcd. for $\mathrm{C}_{30} \mathrm{H}_{34} \mathrm{NaO}_{2} \mathrm{Si}$ $\left([\mathrm{M}+\mathrm{Na}]^{+}\right):$477.2231, Found: 477.2223.

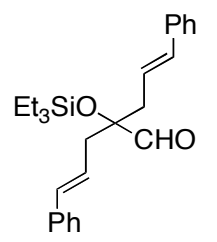

\section{5-Phenyl-2-(3-phenyl-2-propenyl)-2-triethylsiloxy-4-pentenal (1f)}

${ }^{1} \mathrm{H}$ NMR (400 MHz, $\mathrm{CDCl}_{3}$ ) $\delta 9.65$ (1H, s, CHO), 7.20-7.34 (10H, m, Ph-

H), $6.43(2 \mathrm{H}, \mathrm{d}, J=16.0 \mathrm{~Hz}, \mathrm{PhCH}), 6.19(2 \mathrm{H}, \mathrm{dt}, J=16.0,7.6 \mathrm{~Hz}$,

$\mathrm{PhCH}=\mathrm{C} \underline{\mathrm{H}}), 2.60\left(2 \mathrm{H}, \mathrm{dd}, J=7.6,1.2 \mathrm{~Hz}, \mathrm{CH}=\mathrm{CHCH}_{2}\right), 2.58(2 \mathrm{H}, \mathrm{dd}, J$ $\left.=7.6,1.2 \mathrm{~Hz}, \mathrm{CH}=\mathrm{CHCH}_{2}\right), 0.99\left(9 \mathrm{H}, \mathrm{t}, J=8.0 \mathrm{~Hz}, \mathrm{CH}_{3} \mathrm{CH}_{2} \mathrm{Si}\right), 0.67(6 \mathrm{H}, \mathrm{q}, J=8.0 \mathrm{~Hz}$, $\left.\mathrm{CH}_{3} \mathrm{CH}_{2} \mathrm{Si}\right) ;{ }^{13} \mathrm{C}$ NMR $\left(100 \mathrm{MHz}, \mathrm{CDCl}_{3}\right) \delta 203.7,137.2,133.9,128.5,127.3,126.1$, 123.5, 82.6, 40.3, 7.0, 6.8; IR (neat) 3026, 2954, 2908, 2873, 1735, 1598, 1494, 1448, 1238, 1145, 1114, 1066, 1002, 966, 740, 727, $692 \mathrm{~cm}^{-1}$; HRMS (ESI-TOF) Calcd. for $\mathrm{C}_{26} \mathrm{H}_{34} \mathrm{NaO}_{2} \mathrm{Si}\left([\mathrm{M}+\mathrm{Na}]^{+}\right)$: 429.2231, Found: 429.2228.

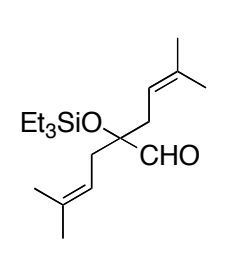

\section{5-Methyl-2-(3-methyl-2-butenyl)-3-triethylsiloxy-4-hexenal (1g)}

${ }^{1} \mathrm{H}$ NMR (400 MHz, $\left.\mathrm{CDCl}_{3}\right) \delta 9.54(1 \mathrm{H}, \mathrm{s}, \mathrm{CHO}), 5.09-5.13(2 \mathrm{H}, \mathrm{m}$,

$\left.\mathrm{Me}_{2} \mathrm{C}=\mathrm{CH}\right), 2.27-2.37\left(4 \mathrm{H}, \mathrm{m}, \mathrm{C}=\mathrm{CHCH}_{2}\right), 1.69\left(3 \mathrm{H}, \mathrm{s}, \mathrm{Me}_{2} \mathrm{C}=\right), 1.58(3 \mathrm{H}$, $\left.\mathrm{s}, \mathrm{Me}_{2} \mathrm{C}=\right), 0.96\left(9 \mathrm{H}, \mathrm{t}, J=7.6 \mathrm{~Hz}, \mathrm{C}_{3} \mathrm{CH}_{2} \mathrm{Si}\right), 0.62(6 \mathrm{H}, \mathrm{q}, J=7.6 \mathrm{~Hz}$, $\left.\mathrm{CH}_{3} \mathrm{CH}_{2} \mathrm{Si}\right) ;{ }^{13} \mathrm{C} \mathrm{NMR}\left(100 \mathrm{MHz}, \mathrm{CDCl}_{3}\right) \delta 204.3,134.5,117.8,83.2,35.0,25.8,17.9$, 6.9, 6.7; IR (neat) 2956, 2912, 2875, 1737, 1456, 1377, 1240, 1141, 1112, 1091, 1008, 977, $727 \mathrm{~cm}^{-1}$; HRMS (ESI-TOF) Calcd. for $\mathrm{C}_{18} \mathrm{H}_{34} \mathrm{NaO}_{2} \mathrm{Si}\left([\mathrm{M}+\mathrm{Na}]^{+}\right): 333.2231$, Found: 333.2219.

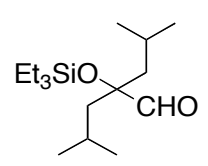

\section{2-Isobutyl-4-methyl-2-triethylsiloxypentanal (1h)}

${ }^{1} \mathrm{H}$ NMR (400 MHz, $\left.\mathrm{CDCl}_{3}\right) \delta 9.57(1 \mathrm{H}, \mathrm{s}, \mathrm{CHO}), 1.72-1.85$ (2H, m, $\left.\mathrm{Me}_{2} \mathrm{CHCH}_{2}\right), 1.51-1.62\left(4 \mathrm{H}, \mathrm{m}, \mathrm{Me}_{2} \mathrm{CHCH}_{2}\right), 0.96(9 \mathrm{H}, \mathrm{t}, J=8.0 \mathrm{~Hz}$, $\left.\mathrm{C}_{3} \mathrm{CH}_{2} \mathrm{Si}\right), 0.90\left(3 \mathrm{H}, \mathrm{d}, J=6.8 \mathrm{~Hz}, \underline{\mathrm{Me}}_{2} \mathrm{CH}\right), 0.86\left(3 \mathrm{H}, \mathrm{d}, J=6.8 \mathrm{~Hz}, \underline{\mathrm{Me}}_{2} \mathrm{CH}\right), 0.65$ $\left(6 \mathrm{H}, \mathrm{q}, J=8.0 \mathrm{~Hz}, \mathrm{CH}_{3} \mathrm{C}_{2} \mathrm{Si}\right) ;{ }^{13} \mathrm{C} \mathrm{NMR}\left(100 \mathrm{MHz}, \mathrm{CDCl}_{3}\right) \delta$ 204.0, 83.7, 46.2, 24.5, 24.0, 23.6, 7.1, 7.0; IR (neat) 2954, 2910, 2875, 1737, 1458, 1186, 1165, 1101, 1008, 977, $727 \mathrm{~cm}^{-1}$; HRMS (ESI-TOF) Calcd. for $\mathrm{C}_{16} \mathrm{H}_{34} \mathrm{NaO}_{2} \mathrm{Si}\left([\mathrm{M}+\mathrm{Na}]^{+}\right)$: 309.2231, Found: 309.2216. 


\section{2,2-Dicyclohexyl-2-triethylsiloxyethanal (1i)}

${ }^{1} \mathrm{H}$ NMR (400 MHz, $\left.\mathrm{CDCl}_{3}\right) \delta 9.58(1 \mathrm{H}, \mathrm{s}, \mathrm{CHO}), 1.73-1.80(8 \mathrm{H}, \mathrm{m},-$ CH-), 1.63-1.66 (6H, m, -CH-), 1.50-1.54 (2H, m, -CH-), 0.95-1.21 (10H, m, - $\mathrm{CH}-), 0.96\left(9 \mathrm{H}, \mathrm{t}, J=8.0 \mathrm{~Hz}, \mathrm{C}_{3} \mathrm{CH}_{2} \mathrm{Si}\right), 0.68\left(6 \mathrm{H}, \mathrm{q}, J=8.0 \mathrm{~Hz}, \mathrm{CH}_{3} \mathrm{C}_{2} \mathrm{Si}\right) ;{ }^{13} \mathrm{C}$ NMR (100 MHz, $\left.\mathrm{CDCl}_{3}\right) \delta 206.5,88.4,42.4,27.9,26.7,26.5,26.4,26.3,7.4,7.3$; IR (neat) 2931, 2873, 2852, 1737, 1448, 1238, 1166, 1147, 1008, $734 \mathrm{~cm}^{-1}$; HRMS (ESITOF) Calcd. for $\mathrm{C}_{20} \mathrm{H}_{38} \mathrm{NaO}_{2} \mathrm{Si}\left([\mathrm{M}+\mathrm{Na}]^{+}\right)$: 361.2533, Found: 361.2537.

III. General Procedure for Catalytic Asymmetric Rearrangement of $\alpha, \alpha$-Dialkyl- $\alpha$ siloxy Aldehydes to Optically Active Acyloins.

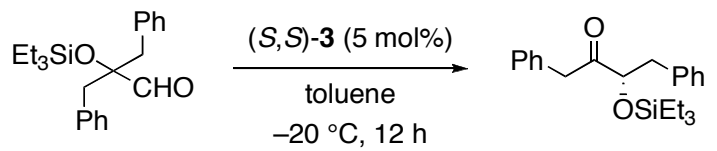

To a solution of 2-hydroxy-2'-[3,5-bis(trifluoromethyl)]phenyl-3-\{2-[3,5bis(trifluoromethyl)]benzenesulfonylamino\}phenyl-1,1'-binaphthyl (4, $46.7 \mathrm{mg}, 0.055$ $\mathrm{mmol})$ in toluene $(10 \mathrm{~mL})$ was added a $1 \mathrm{M}$ toluene solution of trimethylaluminum (50 $\mu \mathrm{L}, 0.050 \mathrm{mmol}$ ) at room temperature under nitrogen and stirred for $30 \mathrm{~min}$. After being cooled to $-20^{\circ} \mathrm{C}$, $1 \mathbf{a}\left(\mathrm{R}^{2}=\mathrm{Et}\right)(354.5 \mathrm{mg}, 1 \mathrm{mmol})$ was added to this solution and the stirring was maintained at $-20^{\circ} \mathrm{C}$ for $12 \mathrm{~h}$. Then, sodium fluoride $(8.4 \mathrm{mg}, 0.20 \mathrm{mmol})$ and water $(2.7 \mu \mathrm{L}, 0.15 \mathrm{mmol})$ were added and the whole mixture was stirred for 30 min at room temperature. To remove precipitates, filtration through celite with EtOAc was carried out. Concentration of the filtrate and purification by column chromatography on silica gel $\left(\mathrm{CH}_{2} \mathrm{Cl} /\right.$ hexane $=1 / 4$ as eluant $)$ afforded $\mathbf{2 a}\left(\mathrm{R}^{2}=\mathrm{Et}\right)$ (342.9 mg, $0.97 \mathrm{mmol}, 97 \%$ yield) as colorless oil. 
IV. Catalytic Asymmetric Rearrangement with Chiral Organoaluminum Catalyst Prepared from Other Ligands

$$
\begin{aligned}
& \mathrm{Me}_{3} \mathrm{Al} \underset{\text { chiral ligand ( } 1.1 \text { equiv) }}{\stackrel{\text { touene }}{\text { r.t., } 30 \text { min }}} \text { Al catalyst }
\end{aligned}
$$

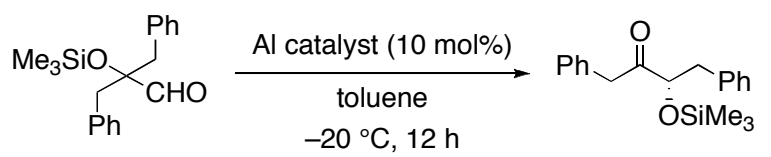

(

${ }^{a}$ Isolated yield.

${ }^{b}$ Enantiomeric excess was determined by HPLC analysis using a chiral column. 


\section{Preparation of Chiral Ligand 9b}

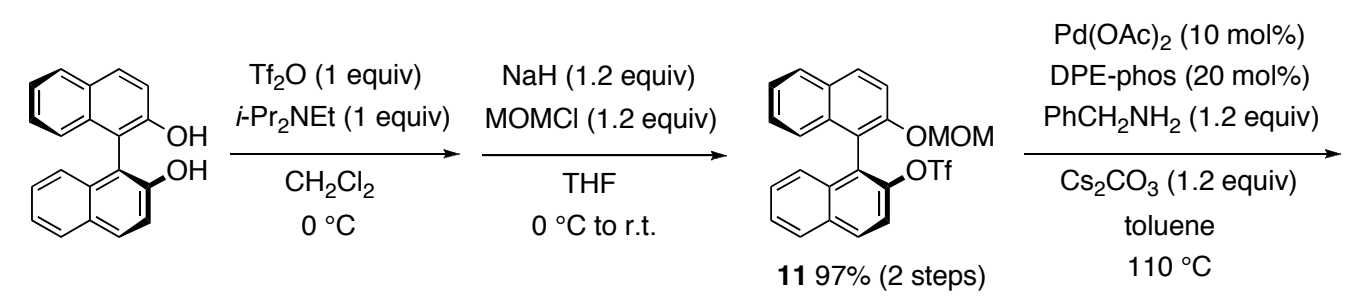

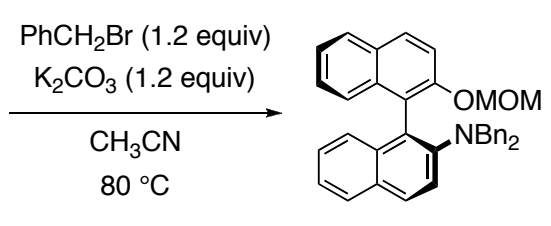

12 91\% (2 steps)

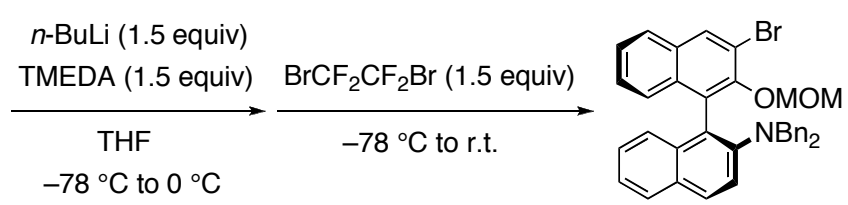

$1390 \%$

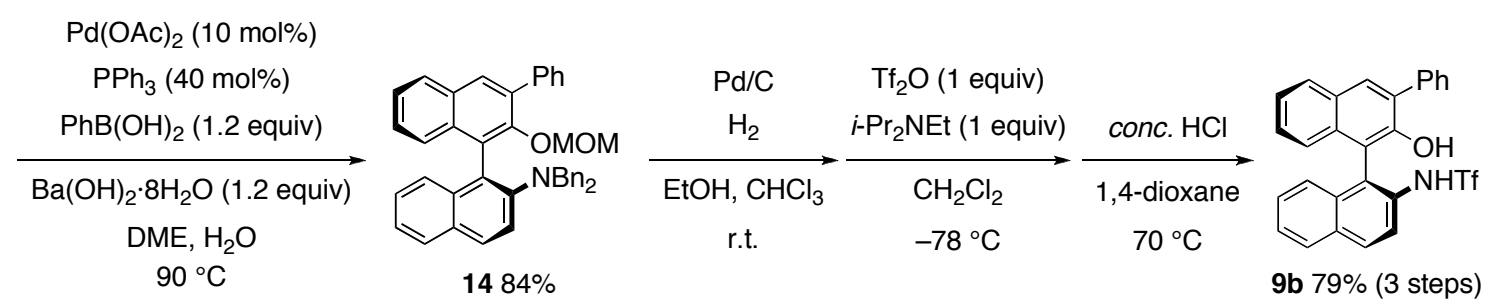

\section{2-Methoxymethoxy-2'-trifluoromethanesulfoxy-1,1'-binaphthyl (11)}

To a solution of $(S)$-binaphthol $(5.73 \mathrm{~g}, 20 \mathrm{mmol})$ and $i$ - $\operatorname{Pr}_{2} \mathrm{NEt}(3.48 \mathrm{~mL}, 20 \mathrm{mmol})$ in $\mathrm{CH}_{2} \mathrm{Cl}_{2}(100 \mathrm{~mL})$ was added $\mathrm{Tf}_{2} \mathrm{O}(3.36 \mathrm{~mL}, 20 \mathrm{mmol})$ at $0{ }^{\circ} \mathrm{C}$ under argon, and the reaction mixture was stirred for $30 \mathrm{~min}$ and quenched with saturated $\mathrm{NaHCO}_{3}$. Extractive workup was performed with $\mathrm{CH}_{2} \mathrm{Cl}_{2}$, and the combined extracts were washed with brine and dried over anhydrous $\mathrm{Na}_{2} \mathrm{SO}_{4}$. After evaporation of solvents and drying under vacuum, the crude products were dissolved into THF $(80 \mathrm{~mL})$ under argon. To this solution was added $\mathrm{NaH}(0.96 \mathrm{~g} ; 60 \%$ dispersion in mineral oil, $24 \mathrm{mmol})$ at $0{ }^{\circ} \mathrm{C}$, and the reaction mixture was maintained at the same temperature for $15 \mathrm{~min}$ with stirring. Chloromethyl methyl ether $(1.82 \mathrm{~mL}, 24 \mathrm{mmol})$ was added dropwise to the solution at $0{ }^{\circ} \mathrm{C}$, and stirring was continued for $3 \mathrm{~h}$ at room temperature. The reaction was quenched with water and extractive workup was conducted with EtOAc. The organic extracts were washed with brine and dried over anhydrous $\mathrm{Na}_{2} \mathrm{SO}_{4}$. Removal of solvents and purification of the residual oil by column chromatography on silica gel $($ EtOAc/hexane $=1 / 10$ as eluant $)$ gave $11(8.97 \mathrm{~g}, 19.4 \mathrm{mmol}, 97 \%$ yield $)$ as white powder. $\quad{ }^{1} \mathrm{H} \mathrm{NMR}\left(400 \mathrm{MHz}, \mathrm{CDCl}_{3}\right) \delta 8.05(1 \mathrm{H}, \mathrm{d}, J=9.2 \mathrm{~Hz}, \mathrm{Ar}-\mathrm{H}), 8.02(1 \mathrm{H}, \mathrm{d}, J$ = 9.6 Hz, Ar-H), $7.98(1 \mathrm{H}, \mathrm{d}, J=8.4 \mathrm{~Hz}, \mathrm{Ar}-\mathrm{H}), 7.89(1 \mathrm{H}, \mathrm{d}, J=8.0 \mathrm{~Hz}, \mathrm{Ar}-\mathrm{H}), 7.63$ $(1 \mathrm{H}, \mathrm{d}, J=9.2 \mathrm{~Hz}, \mathrm{Ar}-\mathrm{H}), 7.57$ (1H, d, $J=8.8 \mathrm{~Hz}, \mathrm{Ar}-\mathrm{H}), 7.54$ (1H, m, Ar-H), 7.34-7.38 (3H, m, Ar-H), 7.25 (1H, m, Ar-H), 7.03 (1H, d, $J=8.4 \mathrm{~Hz}, \mathrm{Ar}-\mathrm{H}), 5.19(1 \mathrm{H}, \mathrm{d}, J=7.2$ $\left.\mathrm{Hz}, \mathrm{ArOCH}_{2}\right), 5.03\left(1 \mathrm{H}, \mathrm{d}, J=7.2 \mathrm{~Hz}, \mathrm{ArOCH}_{2}\right), 3.22\left(3 \mathrm{H}, \mathrm{s}, \mathrm{OCH}_{3}\right)$. 


\section{2-Methoxymethoxy-2'-( $N, N$-dibenzyl)amino-1,1'-binaphthyl (12)}

To a mixture of palladium acetate (436 mg, $1.94 \mathrm{mmol})$, DPE-phos (2.09 g, 3.88 mmol) and cesium carbonate $(7.59 \mathrm{~g}, 23.3 \mathrm{mmol})$ was added a solution of $\mathbf{1 1}(8.97 \mathrm{~g}$, $19.4 \mathrm{mmol})$ and benzylamine $(2.55 \mathrm{~mL}, 23.3 \mathrm{mmol})$ in toluene under argon, and the reaction mixture was heated at $110{ }^{\circ} \mathrm{C}$ for $12 \mathrm{~h}$ with stirring. After cooling to room temperature, the solution was filtered off and the filtrate was washed with brine. The organic layer was dried over $\mathrm{Na}_{2} \mathrm{SO}_{4}$ and concentrated. A mixture of the crude products, benzyl bromide $(2.53 \mathrm{~mL}, 21.2 \mathrm{mmol})$ and potassium carbonate $(2.93 \mathrm{~g}, 21.2 \mathrm{mmol})$ in $\mathrm{CH}_{3} \mathrm{CN}$ was stirred at $80{ }^{\circ} \mathrm{C}$ for $3 \mathrm{~h}$, and then poured into water and extracted with ether. The organic extracts were washed with brine and dried over anhydrous $\mathrm{Na}_{2} \mathrm{SO}_{4}$. Removal of solvents and purification of the residual oil by column chromatography on silica gel (EtOAc/hexane = 1/10 as eluant) afforded $12(10.4 \mathrm{~g}, 17.7 \mathrm{mmol}, 91 \%$ yield $)$ as yellow powder. ${ }^{1} \mathrm{H}$ NMR $\left(400 \mathrm{MHz}, \mathrm{CDCl}_{3}\right) \delta 7.95(1 \mathrm{H}, \mathrm{d}, J=9.2 \mathrm{~Hz}, \mathrm{Ar}-\mathrm{H})$, $7.90(1 \mathrm{H}, \mathrm{d}, J=8.0 \mathrm{~Hz}, \mathrm{Ar}-\mathrm{H}), 7.83(2 \mathrm{H}, \mathrm{d}, J=9.2 \mathrm{~Hz}, \mathrm{Ar}-\mathrm{H}), 7.58(1 \mathrm{H}, \mathrm{d}, J=9.2 \mathrm{~Hz}$, Ar-H), 7.02-7.38 (13H, m, Ar-H), 6.77-6.79 (4H, m, Ar-H), 5.07 (1H, d, J = 7.0 Hz, $\left.\mathrm{ArOCH}_{2}\right), 4.98\left(1 \mathrm{H}, \mathrm{d}, J=7.0 \mathrm{~Hz}, \mathrm{ArOCH}_{2}\right), 4.01\left(2 \mathrm{H}, \mathrm{d}, J=14.6 \mathrm{~Hz}, \mathrm{NCH}_{2} \mathrm{Ph}\right), 3.88$ $\left(2 \mathrm{H}, \mathrm{d}, J=14.6 \mathrm{~Hz}, \mathrm{NCH}_{2} \mathrm{Ph}\right), 3.09\left(3 \mathrm{H}, \mathrm{s}, \mathrm{OCH}_{3}\right)$.

\section{2-Methoxymethoxy-3-bromo-2'-( $N, N$-dibenzyl)amino-1,1'-binaphthyl (13)}

To a solution of $n$-BuLi (17.7 mL; 1.5 M hexane solution, $26.6 \mathrm{mmol})$ in THF (30 mL) was added $N, N, N^{\prime}, N^{\prime}$,-tetramethylethylenediamine $(4.01 \mathrm{~mL}, 26.6 \mathrm{mmol})$ at $-78{ }^{\circ} \mathrm{C}$ under argon. The mixture was stirred for $15 \mathrm{~min}$ and transferred into a solution of $\mathbf{1 2}$ $(10.4 \mathrm{~g}, 17.7 \mathrm{mmol})$ in THF $(50 \mathrm{~mL})$ at the same temperature. After stirring for $3 \mathrm{~h}$ at $0{ }^{\circ} \mathrm{C}$, the resulting mixture was cooled to $-78{ }^{\circ} \mathrm{C}$ again and 1,2-dibromo-1,1,2,2,tetrafluoroethane $(3.16 \mathrm{~mL}, 26.6 \mathrm{mmol})$ was added slowly. This whole reaction mixture was stirred overnight at room temperature. The reaction was quenched with water and extractive workup was performed with ether. The ethereal extracts were washed with brine and dried over anhydrous $\mathrm{Na}_{2} \mathrm{SO}_{4}$. Removal of solvents and purification of the residual oil by column chromatography on silica gel (EtOAc/hexane $=1 / 10$ as eluant) furnished 13 (9.36 g, $15.9 \mathrm{mmol}, 90 \%$ yield) as yellow powder. ${ }^{1} \mathrm{H}$ NMR (400 MHz, $\left.\mathrm{CDCl}_{3}\right) \delta 8.19(1 \mathrm{H}, \mathrm{s}, \mathrm{Ar}-\mathrm{H}), 7.83(2 \mathrm{H}, \mathrm{t}, J=8.4 \mathrm{~Hz}, \mathrm{Ar}-\mathrm{H}), 7.76(1 \mathrm{H}, \mathrm{d}, J=8.0 \mathrm{~Hz}, \mathrm{Ar}-$ H), $7.35(2 \mathrm{H}, \mathrm{t}, J=7.2 \mathrm{~Hz}, \mathrm{Ar}-\mathrm{H}), 7.31(1 \mathrm{H}, \mathrm{d}, J=7.2 \mathrm{~Hz}, \mathrm{Ar}-\mathrm{H}), 7.13-7.25(10 \mathrm{H}, \mathrm{m}$, Ar-H), 6.97-7.00 (4H, m, Ar-H), $4.74\left(1 \mathrm{H}, \mathrm{d}, J=5.4 \mathrm{~Hz}, \mathrm{ArOCH}_{2}\right), 4.52(1 \mathrm{H}, \mathrm{d}, J=5.4$ $\left.\mathrm{Hz}, \mathrm{ArOCH}_{2}\right), 3.80\left(2 \mathrm{H}, \mathrm{d}, J=14.4 \mathrm{~Hz}, \mathrm{NCH}_{2} \mathrm{Ph}\right), 3.78\left(2 \mathrm{H}, \mathrm{d}, J=14.4 \mathrm{~Hz}, \mathrm{NCH}_{2} \mathrm{Ph}\right)$, $2.54\left(3 \mathrm{H}, \mathrm{s}, \mathrm{OCH}_{3}\right)$.

\section{2-Methoxymethoxy-3-phenyl-2'-( $N, N$-dibenzyl)amino-1,1'-binaphthyl (14)}

A mixture of 13 (9.36 g, $15.9 \mathrm{mmol})$, phenylboronic acid (2.32 g, $19.0 \mathrm{mmol})$, barium 
hydroxide octahydrate $(5.99 \mathrm{~g}, 19.0 \mathrm{mmol})$, palladium acetate $(426.5 \mathrm{mg}, 1.9 \mathrm{mmol})$ and triphenylphosphine $(1.99 \mathrm{~g}, 7.6 \mathrm{mmol})$ in DME $(84 \mathrm{~mL})$ and $\mathrm{H}_{2} \mathrm{O}(14 \mathrm{~mL})$ was refluxed for $10 \mathrm{~h}$ with stirring under argon. After cooling to room temperature, the solution was filtered off and the filtrate was washed with brine. The organic layer was dried over anhydrous $\mathrm{Na}_{2} \mathrm{SO}_{4}$. Removal of solvents and purification of the residual oil by column chromatography on silica gel (EtOAc/hexane $=1 / 15$ as eluant) afforded $\mathbf{1 4}$ (7.79 g, $13.3 \mathrm{mmol}, 84 \%$ yield) as yellow powder. ${ }^{1} \mathrm{H} \mathrm{NMR}\left(400 \mathrm{MHz}, \mathrm{CDCl}_{3}\right) \delta 7.92$ $(1 \mathrm{H}, \mathrm{s}, \mathrm{Ar}-\mathrm{H}), 7.88(1 \mathrm{H}, \mathrm{d}, J=8.4 \mathrm{~Hz}, \mathrm{Ar}-\mathrm{H}), 7.84(1 \mathrm{H}, \mathrm{d}, J=8.8 \mathrm{~Hz}, \mathrm{Ar}-\mathrm{H}), 7.80(1 \mathrm{H}$, $\mathrm{d}, J=8.0 \mathrm{~Hz}, \mathrm{Ar}-\mathrm{H})$, 7.63-7.65 (2H, m, Ar-H), 7.30-7.45 (6H, m, Ar-H), 7.08-7.25 (9H, m, Ar-H), 7.03-7.04 (5H, m, Ar-H), $4.21\left(1 \mathrm{H}, \mathrm{d}, J=5.6 \mathrm{~Hz}, \mathrm{ArOCH}_{2}\right), 4.15(1 \mathrm{H}, \mathrm{d}, J=$ $\left.5.6 \mathrm{~Hz}, \mathrm{ArOCH}_{2}\right), 4.14\left(2 \mathrm{H}, \mathrm{d}, J=14.6 \mathrm{~Hz}, \mathrm{NCH}_{2} \mathrm{Ph}\right), 2.11(2 \mathrm{H}, \mathrm{d}, J=14.6 \mathrm{~Hz}$, $\left.\mathrm{NCH}_{2} \mathrm{Ph}\right), 2.54\left(3 \mathrm{H}, \mathrm{s}, \mathrm{OCH}_{3}\right)$.

\section{Chiral Ligand 9b}

A mixture of 14 (7.79 g, $13.3 \mathrm{mmol})$ and palladium-activated carbon (1.4 g; Pd 10\%, $1.3 \mathrm{mmol})$ in $\mathrm{EtOH}(40 \mathrm{~mL})$ and $\mathrm{CHCl}_{3}(10 \mathrm{~mL})$ was stirred for $5 \mathrm{~h}$ at room temperature under $\mathrm{H}_{2}(1 \mathrm{~atm})$. The mixture was filtered off, and the filtrate was concentrated and dried under vacuum. To a solution of the crude products $(5.81 \mathrm{~g}, 9.4 \mathrm{mmol})$ and $i-\mathrm{Pr}_{2} \mathrm{~N}$ Et $(3.28 \mathrm{~mL}, 18.8 \mathrm{mmol})$ in $\mathrm{CH}_{2} \mathrm{Cl}_{2}(30 \mathrm{~mL})$ was added $\mathrm{Tf}_{2} \mathrm{O}(1.58 \mathrm{~mL}, 9.4 \mathrm{mmol})$ at $-78{ }^{\circ} \mathrm{C}$ under argon, and the reaction mixture was stirred there for $2 \mathrm{~h}$ and quenched with saturated $\mathrm{NaHCO}_{3}$. Extractive workup was performed with $\mathrm{CH}_{2} \mathrm{Cl}_{2}$, and the combined extracts were washed with brine and dried over anhydrous $\mathrm{Na}_{2} \mathrm{SO}_{4}$. After evaporation of solvents and drying under vacuum, the crude products were dissolved into 1,4-dioxane $(30 \mathrm{~mL})$ and conc. $\mathrm{HCl}(2-4 \mathrm{~mL})$ was added at room temperature. Then, the mixture was stirred for $5 \mathrm{~h}$ at $70{ }^{\circ} \mathrm{C}$. After cooling to room temperature, the solution was poured into water and extracted with ether. The ethereal extracts were washed with brine and dried over anhydrous $\mathrm{Na}_{2} \mathrm{SO}_{4}$. Removal of solvents and purification of the residual oil by column chromatography on silica gel (EtOAc/hexane $=1 / 5$ as eluant) gave 9b (5.17 g, $10.5 \mathrm{mmol}, 79 \%$ yield). $[\alpha]_{\mathrm{D}}{ }^{25}-20.7^{\circ}$ (c 1.00, $\left.\mathrm{CHCl}_{3}\right) ;{ }^{1} \mathrm{H} \mathrm{NMR}(400$ $\left.\mathrm{MHz}, \mathrm{CDCl}_{3}\right) \delta 8.04(1 \mathrm{H}, \mathrm{d}, J=9.6 \mathrm{~Hz}, \mathrm{Ar}-\mathrm{H}), 7.99(1 \mathrm{H}, \mathrm{s}, \mathrm{Ar}-\mathrm{H}), 7.95(1 \mathrm{H}, \mathrm{d}, J=8.4$ $\mathrm{Hz}, \mathrm{Ar}-\mathrm{H}), 7.92$ (2H, t, $J=8.2 \mathrm{~Hz}, \mathrm{Ar}-\mathrm{H}), 7.63-7.65(2 \mathrm{H}, \mathrm{m}, \mathrm{Ar}-\mathrm{H}), 7.26-7.53$ (8H, m, Ar-H), $6.97(1 \mathrm{H}, \mathrm{d}, J=8.4 \mathrm{~Hz}, \mathrm{Ar}-\mathrm{H}), 6.67(1 \mathrm{H}, \mathrm{br}, \mathrm{NH}), 5.30(1 \mathrm{H}, \mathrm{br}, \mathrm{OH}) ;{ }^{13} \mathrm{C} \mathrm{NMR}$ $\left(100 \mathrm{MHz}, \mathrm{CDCl}_{3}\right) \delta 148.8,136.2,132.6,132.5,131.9,131.7,131.4,130.5,130.3$, 129.2, 129.1, 129.0, 128.5, 128.2, 128.2, 127.6, 127.5, 126.3, 125.8, 124.5, 123.6, 123.3, $119.9,119.3$ (q, $J_{\mathrm{C}-\mathrm{F}}=324 \mathrm{~Hz}$ ), 112.7; IR (neat) 3523, 3321, 3059, 2358, 1705, 1423, 1220, 1197, 1139, 995, 752, 704, $603 \mathrm{~cm}^{-1}$; HRMS (ESI-TOF) Calcd. for $\mathrm{C}_{27} \mathrm{H}_{17} \mathrm{~F}_{3} \mathrm{NO}_{3} \mathrm{~S}$ $\left([\mathrm{M}-\mathrm{H}]^{-}\right)$: 492.0886, Found: 492.0895. 


\section{Representative Procedure for the Synthesis of Chiral Ligand 4 and 10:}

\section{Chiral Ligand 10b}

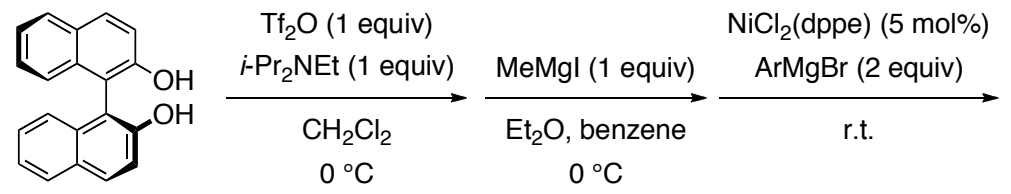

$0^{\circ} \mathrm{C}$

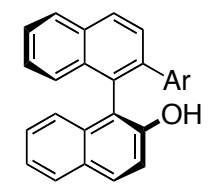

$\left(\mathrm{Ar}=3,5-\left(\mathrm{CF}_{3}\right)_{2} \mathrm{C}_{6} \mathrm{H}_{3}\right)$

15 59\% (2 steps)
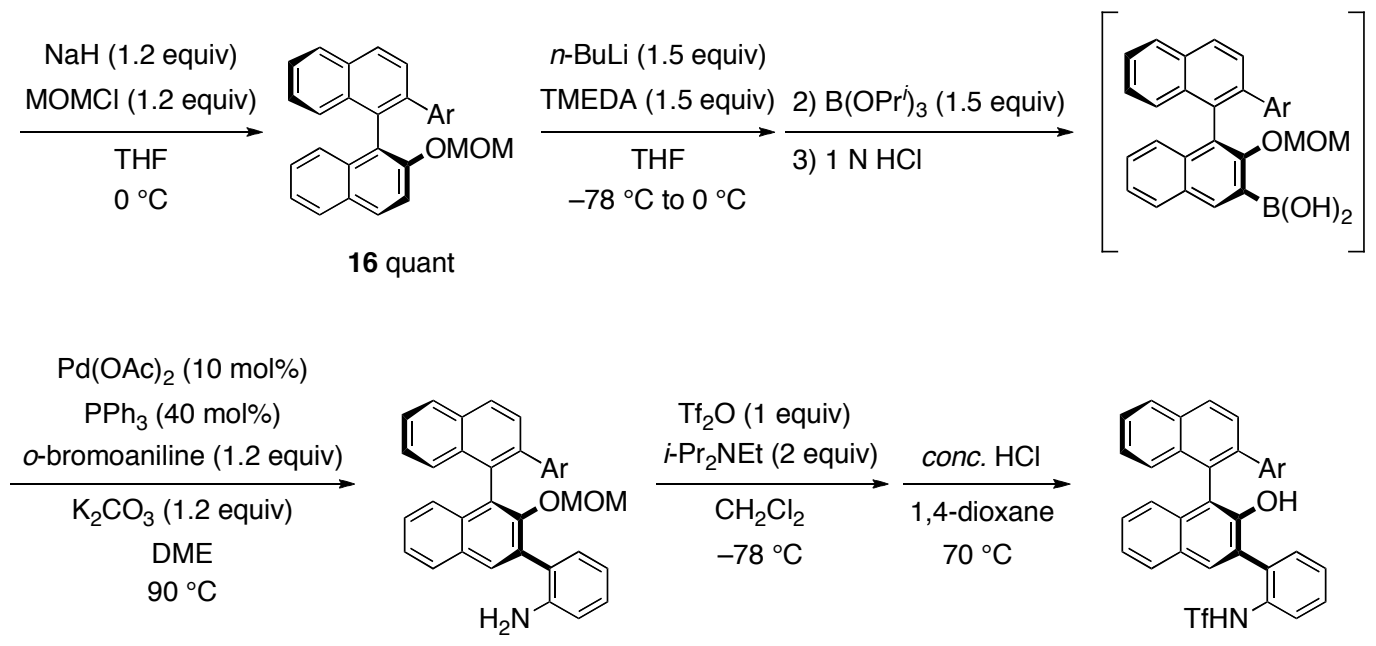
$\mathrm{Tf}_{2} \mathrm{O}$ (1 equiv)
$\underset{\mathrm{CH}_{2} \mathrm{Cl}_{2}}{\stackrel{i-\mathrm{Pr}_{2} \mathrm{NEt}(2 \text { equiv) }}{\longrightarrow}} \stackrel{\text { conc. } \mathrm{HCl}}{\underset{1,4-\text { dioxane }}{\longrightarrow}}$
$-78^{\circ} \mathrm{C}$
$70{ }^{\circ} \mathrm{C}$

$1780 \%$ (2 steps)

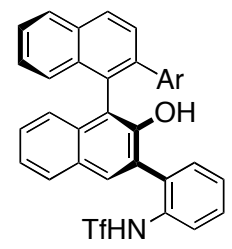

10b $81 \%$ (2 steps)

\section{2-Hydroxy-2'-[3,5-bis(trifluoromethyl)]phenyl-1,1'-binaphthyl (15)}

To a solution of $(S)$-binaphthol $(5.73 \mathrm{~g}, 20 \mathrm{mmol})$ and $i$-Pr $\operatorname{Pr}_{2} \mathrm{NEt}(3.48 \mathrm{~mL}, 20 \mathrm{mmol})$ in $\mathrm{CH}_{2} \mathrm{Cl}_{2}(100 \mathrm{~mL})$ was added $\mathrm{Tf}_{2} \mathrm{O}(3.36 \mathrm{~mL}, 20 \mathrm{mmol})$ at $0{ }^{\circ} \mathrm{C}$ under argon, and the reaction mixture was stirred for $30 \mathrm{~min}$ and quenched with saturated $\mathrm{NaHCO}_{3}$. Extractive workup was performed with $\mathrm{CH}_{2} \mathrm{Cl}_{2}$, and the combined extracts were washed with brine and dried over anhydrous $\mathrm{Na}_{2} \mathrm{SO}_{4}$. After evaporation of solvents and drying under vacuum, the crude product was dissolved into benzene $(40 \mathrm{~mL})$ under argon. To this solution was added a $1 \mathrm{M}$ ether solution of $\mathrm{MeMgI}(20 \mathrm{~mL}, 20 \mathrm{mmol})$ at $0{ }^{\circ} \mathrm{C}$, and the reaction solution was maintained at the same temperature for $15 \mathrm{~min}$ with stirring. The successive addition of $\mathrm{NiCl}_{2}$ (dppe) $(0.53 \mathrm{~g}, 1 \mathrm{mmol})$ and a $1 \mathrm{M}$ ether solution of 3,5-bis(trifluoromethyl)phenyl magnesium bromide was performed at room temperature, and stirring was continued for $1 \mathrm{~h}$ at room temperature. The reaction was quenched with saturated $\mathrm{NH}_{4} \mathrm{Cl}$ carefully and extractive workup was conducted with ether. The organic extracts were washed with brine and dried over anhydrous $\mathrm{Na}_{2} \mathrm{SO}_{4}$. Removal of solvents and purification of the residual oil by column chromatography on silica gel $\left(\mathrm{Et}_{2} \mathrm{O} /\right.$ hexane $=1 / 10$ then $\mathrm{EtOAc} /$ hexane $=1 / 10$ as eluants $)$ afforded 15 (5.70 g, 11.8 mmol, 59\% yield) as yellow powder. ${ }^{1} \mathrm{H}$ NMR $\left(400 \mathrm{MHz}, \mathrm{CDCl}_{3}\right) \delta 8.14(1 \mathrm{H}, \mathrm{d}, J=$ $8.8 \mathrm{~Hz}, \mathrm{Ar}-\mathrm{H}), 8.02(1 \mathrm{H}, \mathrm{d}, J=8.4 \mathrm{~Hz}, \mathrm{Ar}-\mathrm{H}), 7.78(1 \mathrm{H}, \mathrm{d}, J=9.2 \mathrm{~Hz}, \mathrm{Ar}-\mathrm{H}), 7.76(1 \mathrm{H}$, 
d, $J=9.2 \mathrm{~Hz}, \mathrm{Ar}-\mathrm{H}), 7.69$ (1H, d, $J=8.8 \mathrm{~Hz}, \mathrm{Ar}-\mathrm{H}), 7.38-7.59$ (6H, m, Ar-H), 7.19-7.28 $(2 \mathrm{H}, \mathrm{m}, \mathrm{Ar}-\mathrm{H}), 7.13(1 \mathrm{H}, \mathrm{d}, J=8.8 \mathrm{~Hz}, \mathrm{Ar}-\mathrm{H}), 6.97(1 \mathrm{H}, \mathrm{d}, J=8.4 \mathrm{~Hz}, \mathrm{Ar}-\mathrm{H}), 4.74(1 \mathrm{H}$, $\mathrm{s}, \mathrm{HO}) ;{ }^{13} \mathrm{C}$ NMR $\left(100 \mathrm{MHz}, \mathrm{CDCl}_{3}\right) \delta 151.0,142.6,138.1,133.5,132.8,130.6$ (q, $J_{\mathrm{C}-\mathrm{F}}$ $=33.8 \mathrm{~Hz}), 130.4,129.9,129.7,128.7,128.2$, 128.1, 127.6, 127.1, 127.1, 126.9, 126.2, 124.1, 123.4, 122.9 (q, $\left.J_{\mathrm{C}-\mathrm{F}}=274 \mathrm{~Hz}\right), 120.5,120.4,120.4,116.9$, 116.4; HRMS (ESITOF) Calcd. for $\mathrm{C}_{28} \mathrm{H}_{15} \mathrm{~F}_{6} \mathrm{O}\left([\mathrm{M}-\mathrm{H}]^{-}\right)$: 481.1021, Found: 481.1035.

\section{2-Methoxymethoxy-2'-[3,5-bis(trifluoromethyl)]phenyl-1,1'-binaphthyl (16)}

To a solution of $15(5.70 \mathrm{~g}, 11.8 \mathrm{mmol})$ in THF (30 mL) was added $\mathrm{NaH}(5.7 \mathrm{~g} ; 60 \%$ dispersion in mineral oil, $14.1 \mathrm{mmol}$ ) at $0{ }^{\circ} \mathrm{C}$ carefully, and the mixture was stirred for $15 \mathrm{~min}$. Chloromethyl methyl ether $(1.07 \mathrm{~mL}, 14.1 \mathrm{mmol})$ was added dropwise to the mixture at $0{ }^{\circ} \mathrm{C}$, and the whole reaction mixture was then stirred for $1 \mathrm{~h}$ at room temperature. The mixture was quenched with water and extracted with ether. The organic extracts were washed with brine and dried over anhydrous $\mathrm{Na}_{2} \mathrm{SO}_{4}$. Removal of solvents and purification of the residual oil by short column chromatography on silica gel $($ EtOAc/hexane $=1 / 10$ as eluant $)$ gave $16(6.22 \mathrm{~g}, 11.8 \mathrm{mmol}$, quant $)$ as white powder. $\quad{ }^{1} \mathrm{H}$ NMR $\left(400 \mathrm{MHz}, \mathrm{CDCl}_{3}\right) \delta 8.07(1 \mathrm{H}, \mathrm{d}, J=8.8 \mathrm{~Hz}, \mathrm{Ar}-\mathrm{H}), 7.98(1 \mathrm{H}, \mathrm{d}, J$ $=8.0 \mathrm{~Hz}, \mathrm{Ar}-\mathrm{H}), 7.83(1 \mathrm{H}, \mathrm{d}, J=9.2 \mathrm{~Hz}, \mathrm{Ar}-\mathrm{H}), 7.76(1 \mathrm{H}, \mathrm{d}, J=8.0 \mathrm{~Hz}, \mathrm{Ar}-\mathrm{H}), 7.64$ $(1 \mathrm{H}, \mathrm{d}, J=8.4 \mathrm{~Hz}, \mathrm{Ar}-\mathrm{H}), 7.59(2 \mathrm{H}, \mathrm{s}, \mathrm{Ar}-\mathrm{H}), 7.49-7.51(2 \mathrm{H}, \mathrm{m}, \mathrm{Ar}-\mathrm{H}), 7.43(1 \mathrm{H}, \mathrm{d}, J=$ $9.2 \mathrm{~Hz}, \mathrm{Ar}-\mathrm{H}), 7.19-7.33$ (4H, m, Ar-H), $7.00(1 \mathrm{H}, \mathrm{d}, J=8.0 \mathrm{~Hz}, \mathrm{Ar}-\mathrm{H}), 4.98(1 \mathrm{H}, \mathrm{d}, J=$ $\left.6.8 \mathrm{~Hz}, \mathrm{OCH}_{2} \mathrm{O}\right), 4.92\left(1 \mathrm{H}, \mathrm{d}, J=6.8 \mathrm{~Hz}, \mathrm{OCH}_{2} \mathrm{O}\right), 3.12\left(3 \mathrm{H}, \mathrm{s}, \mathrm{OCH}_{3}\right) ;{ }^{13} \mathrm{C} \mathrm{NMR}(100$ $\left.\mathrm{MHz} \mathrm{CDCl}_{3}\right) \delta 152.8,143.7,136.7,133.7,133.2,132.9,130.3\left(\mathrm{q}, J_{\mathrm{C}-\mathrm{F}}=33.0 \mathrm{~Hz}\right.$ ), $130.0,129.2,128.9,128.8,128.4,127.9,126.7,126.7,126.6,126.3,124.7,123.9,123.0$ $\left(\mathrm{q}, J_{\mathrm{C}-\mathrm{F}}=274 \mathrm{~Hz}\right), 121.2,120.0,119.9,119.9,115.4$, 94.6, 55.7; HRMS (ESI-TOF) Calcd. for $\mathrm{C}_{30} \mathrm{H}_{20} \mathrm{~F}_{6} \mathrm{NaO}_{2}\left([\mathrm{M}+\mathrm{Na}]^{+}\right)$: 549.1259, Found: 549.1277.

\section{2-Methoxymethoxy-2'-[3,5-bis(trifluoromethyl)]phenyl-3-(2-amino)phenyl-1,1'- binaphthyl (17)}

To a solution of $N, N, N^{\prime}, N^{\prime}$-tetramethylethylenediamine $(2.67 \mathrm{~mL}, 17.7 \mathrm{mmol})$ in THF $(30 \mathrm{~mL})$ was added a $1.5 \mathrm{M}$ hexane solution of $n$-BuLi $(11.8 \mathrm{~mL}, 17.7 \mathrm{mmol})$ at $-78{ }^{\circ} \mathrm{C}$ under argon, and the mixture was stirred for $30 \mathrm{~min}$. A solution of $\mathbf{1 6}(6.22 \mathrm{~g}, 11.8$ mmol) in THF $(10 \mathrm{~mL})$ was added to this mixture at $-78{ }^{\circ} \mathrm{C}$ and the resulting mixture was kept at $-78{ }^{\circ} \mathrm{C}$ for $30 \mathrm{~min}$ and at $0{ }^{\circ} \mathrm{C}$ for $5 \mathrm{~h}$ with stirring. The reaction mixture was cooled to $-78{ }^{\circ} \mathrm{C}$ again and transferred into a solution of triisopropyl borate (4.08 $\mathrm{mL}, 17.7 \mathrm{mmol})$ in THF $(10 \mathrm{~mL})$. After stirring for $1 \mathrm{~h}$ at $-78{ }^{\circ} \mathrm{C}$, the solution was stirred overnight at room temperature. $1 \mathrm{~N} \mathrm{HCl}$ was added and the mixture was stirred vigorously for another $1 \mathrm{~h}$. Then extractive workup was performed with ether, and the ethereal extracts were washed with brine and dried over anhydrous $\mathrm{Na}_{2} \mathrm{SO}_{4}$. After 
evaporation of solvents, the crude products were dried under vacuum. A mixture of the crude products, $o$-bromoaniline $(2.43 \mathrm{~g}, 14.1 \mathrm{mmol})$, potassium carbonate $(1.95 \mathrm{~g}, 14.1$ mmol), palladium acetate $(264.9 \mathrm{mg}, 1.18 \mathrm{mmol})$ and triphenylphosphine $(1.24 \mathrm{~g}, 4.72$ mmol) in dried DME (45 mL) was refluxed for $10 \mathrm{~h}$ with stirring under argon. After cooling to room temperature, the solution was filtered off and the filtrate was washed with brine. The Organic layer was dried over anhydrous $\mathrm{Na}_{2} \mathrm{SO}_{4}$. Removal of solvents and purification of the residual oil by column chromatography on silica gel $($ EtOAc/hexane $=1 / 15$ as eluant $)$ afforded 17 (5.81 g, $9.4 \mathrm{mmol}, 80 \%$ yield $)$ as a mixture of two conformational isomers (2.4:1). These conformers were interconvertible at room temperature. $\quad{ }^{1} \mathrm{H} \mathrm{NMR}\left(400 \mathrm{MHz}, \mathrm{CDCl}_{3}\right.$, major conformer) $\delta 8.07(1 \mathrm{H}, \mathrm{d}, J=$ $8.4 \mathrm{~Hz}, \mathrm{Ar}-\mathrm{H}), 7.98$ (1H, d, $J=8.4 \mathrm{~Hz}, \mathrm{Ar}-\mathrm{H}), 7.87$ (1H, d, $J=8.4 \mathrm{~Hz}, \mathrm{Ar}-\mathrm{H}), 7.80(1 \mathrm{H}$, s, Ar-H), 7.08-7.65 (12H, m, Ar-H), $6.79(1 \mathrm{H}, \mathrm{t}, J=7.2 \mathrm{~Hz}, \mathrm{Ar}-\mathrm{H}), 6.60(1 \mathrm{H}, \mathrm{d}, J=8.0$ $\mathrm{Hz}, \mathrm{Ar}-\mathrm{H}), 4.17\left(2 \mathrm{H}, \mathrm{s}, \mathrm{OCH}_{2} \mathrm{O}\right), 2.85\left(2 \mathrm{H}, \mathrm{br}, \mathrm{NH}_{2}\right), 2.18\left(3 \mathrm{H}, \mathrm{s}, \mathrm{OCH}_{3}\right)$. [minor (detectable peaks)] $\delta 8.07(1 \mathrm{H}, \mathrm{d}, J=8.4 \mathrm{~Hz}, \mathrm{Ar}-\mathrm{H}), 7.98(1 \mathrm{H}, \mathrm{d}, J=8.4 \mathrm{~Hz}, \mathrm{Ar}-\mathrm{H})$, 7.75-7.83 (2H, m, Ar-H), 7.08-7.65 (12H, m, Ar-H), 6.79 (1H, t, J= 7.2 Hz, Ar-H), 6.66 $(1 \mathrm{H}, \mathrm{d}, 6.4 \mathrm{~Hz}, \mathrm{Ar}-\mathrm{H}), 4.34\left(1 \mathrm{H}, \mathrm{d}, 6.0 \mathrm{~Hz}, \mathrm{OCH}_{2} \mathrm{O}\right), 4.23\left(1 \mathrm{H}, \mathrm{d}, 6.0 \mathrm{~Hz}, \mathrm{OCH}_{2} \mathrm{O}\right), 3.83$ $\left(2 \mathrm{H}\right.$, br, $\left.\mathrm{NH}_{2}\right), 2.23\left(3 \mathrm{H}, \mathrm{s}, \mathrm{OCH}_{3}\right) ;{ }^{13} \mathrm{C} \mathrm{NMR}\left(100 \mathrm{MHz}, \mathrm{CDCl}_{3}\right.$, major conformer) $\delta 150.0,144.2$, 144.1, 137.4, 133.8, 133.3, 133.1, 132.2, 131.5, 131.0, 130.6, 130.5 (q, $\left.J_{\mathrm{C}-\mathrm{F}}=33.8 \mathrm{~Hz}\right), 129.0,128.7,128.2,127.9,127.3,127.0,126.8,126.6,126.4,125.5$, $125.3,124.6,123.6,122,9\left(\mathrm{q}, J_{\mathrm{C}-\mathrm{F}}=274 \mathrm{~Hz}\right), 120.1,120.1,120.1,118.2,115.5,98.4$, 55.5. [minor (detectable peaks)] $\delta 151.2,143.8,143.6,133.1,133.0,132.6,131.2,129.2$, $128.6,128.5,127.8,127.4,126.7,126.3,125.4,125.1,123.0$ (q, $\left.J_{\mathrm{C}-\mathrm{F}}=274 \mathrm{~Hz}\right), 119.9$, 118.3, 115.7, 98.2, 65.8; HRMS (ESI-TOF) Calcd. for $\mathrm{C}_{36} \mathrm{H}_{26} \mathrm{~F}_{6} \mathrm{NO}_{2}\left([\mathrm{M}+\mathrm{H}]^{+}\right): 618.1862$, Found: 618.1856.

\section{Chiral Ligand 10b}

To a solution of $17(5.81 \mathrm{~g}, 9.4 \mathrm{mmol})$ and $i-\mathrm{Pr}_{2} \mathrm{NEt}(3.28 \mathrm{~mL}, 18.8 \mathrm{mmol})$ in $\mathrm{CH}_{2} \mathrm{Cl}_{2}$ (30 mL) was added $\mathrm{Tf}_{2} \mathrm{O}(1.58 \mathrm{~mL}, 9.4 \mathrm{mmol})$ at $-78{ }^{\circ} \mathrm{C}$ under argon, and the reaction mixture was stirred for $2 \mathrm{~h}$ and quenched with saturated $\mathrm{NaHCO}_{3}$. Extractive workup was performed with $\mathrm{CH}_{2} \mathrm{Cl}_{2}$, and the combined extracts were washed with brine and dried over anhydrous $\mathrm{Na}_{2} \mathrm{SO}_{4}$. After evaporation of solvents and drying under vacuum, the crude products were dissolved into 1,4-dioxane $(30 \mathrm{~mL})$ and conc. $\mathrm{HCl}(2-4 \mathrm{~mL})$ was added at room temperature. Then, the mixture was stirred for $5 \mathrm{~h}$ at $70{ }^{\circ} \mathrm{C}$. After cooling to room temperature, the solution was poured into water and extracted with ether. The ethereal extracts were washed with brine and dried over anhydrous $\mathrm{Na}_{2} \mathrm{SO}_{4}$. Removal of solvents and purification of the residual oil by column chromatography on silica gel (EtOAc/hexane = 1/5 as eluant) furnished $\mathbf{1 0 b}(5.37 \mathrm{~g}, 7.6 \mathrm{mmol}, 81 \%$ yield $)$. 
Two conformational isomers were detectable at $-40{ }^{\circ} \mathrm{C}\left({ }^{1} \mathrm{H}\right.$ NMR for aromatic and phenol protons, see below).
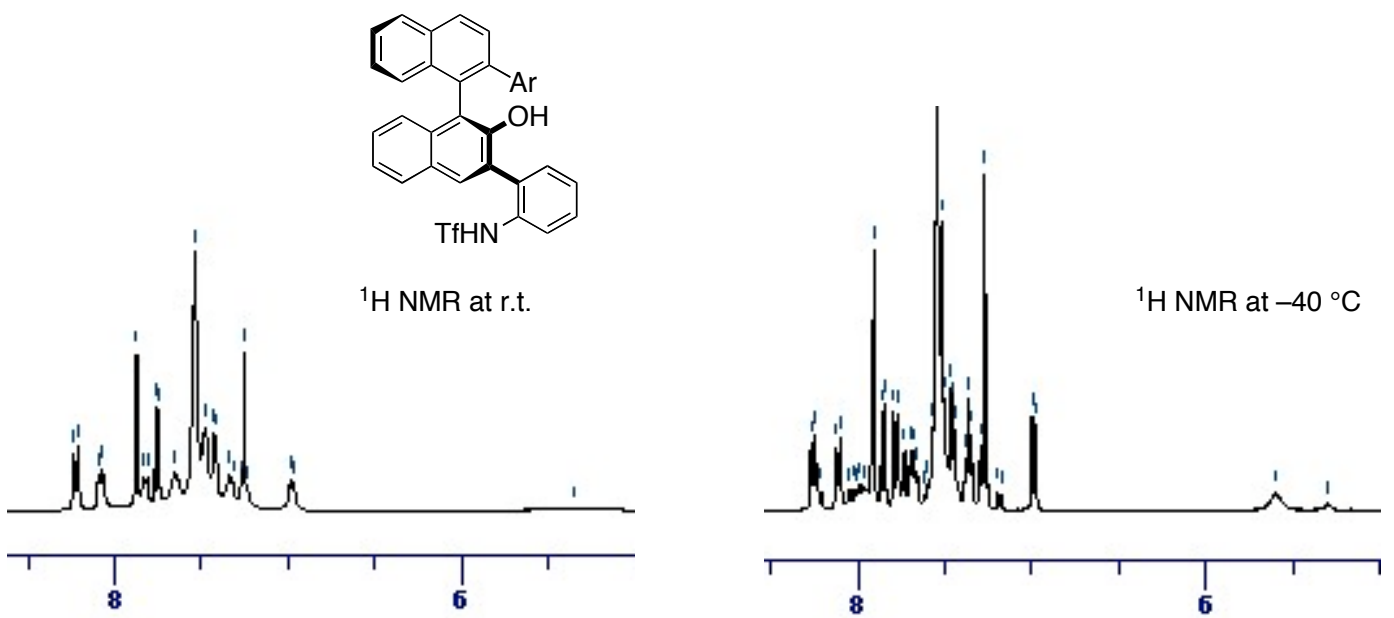

$[\alpha]_{\mathrm{D}}{ }^{26}-46.4^{\circ}\left(c 1.01, \mathrm{CHCl}_{3}\right) ;{ }^{1} \mathrm{H} \mathrm{NMR}\left(400 \mathrm{MHz}, \mathrm{CDCl}_{3}\right) \delta 8.22(1 \mathrm{H}, \mathrm{d}, J=7.6 \mathrm{~Hz}$, Ar-H), $8.08(1 \mathrm{H}, \mathrm{d}, J=8.0 \mathrm{~Hz}, \mathrm{Ar}-\mathrm{H}), 7.87(1 \mathrm{H}, \mathrm{s}, \mathrm{Ar}-\mathrm{H}), 7.82(1 \mathrm{H}, \mathrm{d}, J=7.6 \mathrm{~Hz}, \mathrm{Ar}-\mathrm{H})$, $7.75(1 \mathrm{H}, \mathrm{d}, J=8.8 \mathrm{~Hz}, \mathrm{Ar}-\mathrm{H}), 7.23-7.65(9 \mathrm{H}, \mathrm{m}, \mathrm{Ar}-\mathrm{H}), 5.35(1 \mathrm{H}, \mathrm{br}, \mathrm{OH}) ;{ }^{13} \mathrm{C} \mathrm{NMR}$ $\left(100 \mathrm{MHz}, \mathrm{CDCl}_{3}\right) \delta 147.2,142.3,138.3,134.6,133.6,132.8,132.6,132.5,132.3$, $132.1,131.5,130.7\left(\mathrm{q}, J_{\mathrm{C}-\mathrm{F}}=33.8 \mathrm{~Hz}\right), 130.6,129.1,128.9,128.6,128.5,128.4,128.3$, 127.6, 127.5, 127.1, 126.4, 126.1, 125.5, 125.1, 124.5 (q, $\left.J_{\mathrm{C}-\mathrm{F}}=307 \mathrm{~Hz}\right), 124.1,124.0$, $122.8\left(\mathrm{q}, J_{\mathrm{C}-\mathrm{F}}=274 \mathrm{~Hz}\right), 120.5,117.8$; IR (neat) 3502, 3296, 3062, 2926, 1411, 1386, 1354, 1278, 1230, 1195, 1182, 1136, 896, 823, 752, $603 \mathrm{~cm}^{-1}$; HRMS (ESI-TOF) Calcd. for $\mathrm{C}_{35} \mathrm{H}_{19} \mathrm{~F}_{9} \mathrm{NO}_{3} \mathrm{~S}\left([\mathrm{M}-\mathrm{H}]^{-}\right)$: 704.0947, Found: 704.0951.

Chiral Ligand 10a (synthesized by the same procedure) $[\alpha]_{\mathrm{D}}{ }^{26}-78.6^{\circ}\left(c 1.05, \mathrm{CHCl}_{3}\right) ;{ }^{1} \mathrm{H} \mathrm{NMR}\left(400 \mathrm{MHz}, \mathrm{CDCl}_{3}\right) \delta 8.14(1 \mathrm{H}, \mathrm{d}, J=8.8 \mathrm{~Hz}$, Ar-H), $8.02(1 \mathrm{H}, \mathrm{d}, J=8.0 \mathrm{~Hz}, \mathrm{Ar}-\mathrm{H}), 7.81-7.84(2 \mathrm{H}, \mathrm{m}, \mathrm{Ar}-\mathrm{H}), 7.72(1 \mathrm{H}, \mathrm{d}, 8.4 \mathrm{~Hz})$, 7.26-7.59 (9H, m, Ar-H), 7.03-7.25 (6H, m, Ar-H), 5.23 (1H, br, OH); ${ }^{13} \mathrm{C}$ NMR (100 $\left.\mathrm{MHz}, \mathrm{CDCl}_{3}\right) \delta 146.8,141.8,140.3,135.0,133.9,133.2,132.9,132.3,131.8,130.0$, 129.0, 128.8, 128.6, 128.5, 128.4, 128.3, 128.0, 127.7, 127.3, 127.3, 126.8, 126.6, 126.0, 125.7, 125.1, 124.4, $119.5\left(\mathrm{q}, J_{\mathrm{C}-\mathrm{F}}=324 \mathrm{~Hz}\right), 119.1$ (three signals for aromatic carbons were not identified due to the overlap of the peaks); IR (neat) 3502, 3282, 3057, 2927, 1732, 1494, 1411, 1355, 1230, 1195, 1141, 1028, 952, 825, 765, 750, 700, $603 \mathrm{~cm}^{-1}$; HRMS (ESI-TOF) Calcd. for $\mathrm{C}_{33} \mathrm{H}_{21} \mathrm{~F}_{3} \mathrm{NO}_{3} \mathrm{~S}\left([\mathrm{M}-\mathrm{H}]^{-}\right)$: 568.1188, Found: 568.1191. 
Chiral Ligand $(S, S)-4$
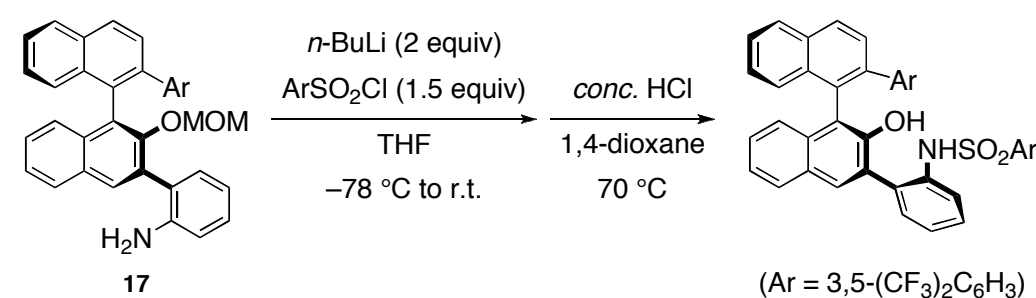

$\left(\mathrm{Ar}=3,5-\left(\mathrm{CF}_{3}\right)_{2} \mathrm{C}_{6} \mathrm{H}_{3}\right)$

$(S, S)-458 \%$ (2 steps)

To a solution of $17(1.23 \mathrm{~g}, 2.0 \mathrm{mmol})$ in THF $(6 \mathrm{~mL})$ was added a $1.5 \mathrm{M}$ hexane solution of $n$-BuLi $(2.67 \mathrm{~mL}, 4.0 \mathrm{mmol})$ slowly at $-78{ }^{\circ} \mathrm{C}$, and the mixture was stirred for $30 \mathrm{~min}$. 3,5-Bis(trifluoromethyl)benzenesulfonyl chloride (937.8 mg, $3.0 \mathrm{mmol}$ ) was added to this mixture, and the stirring was maintained for $30 \mathrm{~min}$ at $-78{ }^{\circ} \mathrm{C}$ and $3 \mathrm{~h}$ at room temperature. The reaction was quenched with saturated $\mathrm{NH}_{4} \mathrm{Cl}$ and extractive workup was conducted with ether. The organic extracts were washed with brine and dried over anhydrous $\mathrm{Na}_{2} \mathrm{SO}_{4}$. After evaporation of solvents and drying under vacuum, the crude products were dissolved into 1,4-dioxane $(10 \mathrm{~mL})$ and conc. $\mathrm{HCl}(1-2 \mathrm{~mL})$ was added at room temperature. Then, the mixture was stirred for $5 \mathrm{~h}$ at $70{ }^{\circ} \mathrm{C}$. After cooling to room temperature, the solution was poured into water and extracted with ether. The ethereal extracts were washed with brine and dried over anhydrous $\mathrm{Na}_{2} \mathrm{SO}_{4}$. Removal of solvents and purification of the residual oil by column chromatography on silica gel (EtOAc/hexane = 1/10 as eluant) afforded stereochemically pure $(S, S)$-4 (1.00 g, 1.16 mmol, 58\% yield). Diastereomeric $(S, S)-4$ and $(S, R)-\mathbf{4}$ were not interconvertible, and these were separable by column chromatography on silica gel (crude product: $\mathrm{dr}=$ 8:1). $[\alpha]_{\mathrm{D}}{ }^{24}+32.2^{\circ}\left(c 1.04, \mathrm{CH}_{3} \mathrm{OH}\right) ;{ }^{1} \mathrm{H} \mathrm{NMR}\left(400 \mathrm{MHz}, \mathrm{CDCl}_{3}\right) \delta 8.22(1 \mathrm{H}, \mathrm{d}, J=4.8$ $\mathrm{Hz}, \mathrm{Ar}-\mathrm{H}), 8.10-8.13$ (1H, m, Ar-H), 7.70-7.74 (7H, m, Ar-H), 7.53 (1H, t, $J=7.8 \mathrm{~Hz}$, Ar-H), 7.40-7.45 (7H, m, Ar-H), 7.17-7.27 (2H, m, Ar-H), 6.96 (1H, s, Ar-H), $6.88(1 \mathrm{H}$, $\mathrm{d}, J=7.6 \mathrm{~Hz}, \mathrm{Ar}-\mathrm{H}), 5.16(1 \mathrm{H}, \mathrm{br}, \mathrm{OH}) ;{ }^{13} \mathrm{C} \mathrm{NMR}\left(100 \mathrm{MHz}, \mathrm{CDCl}_{3}\right) \delta 146.8,142.3$, $141.4,138.4,134.2,133.6,132.4,132.3,132.1$ (q, $\left.J_{\mathrm{C}-\mathrm{F}}=34.6 \mathrm{~Hz}\right), 131.2,131.0,130.5$ $\left(\mathrm{q}, J_{\mathrm{C}-\mathrm{F}}=33.3 \mathrm{~Hz}\right), 130.4,129.4,128.6,128.4,128.2,128.1,127.7,127.5,127.4,127.1$, $126.3,125.9,125.7,124.9,124.7,124.1,122.8\left(\mathrm{q}, J_{\mathrm{C}-\mathrm{F}}=273 \mathrm{~Hz}\right), 121.9\left(\mathrm{q}, J_{\mathrm{C}-\mathrm{F}}=290\right.$ $\mathrm{Hz}$ ), 120.4, 120.4, 120.3, 117.6 (two signals for aromatic carbons were not identified due to the overlap of the peaks); IR (neat) 3298, 2970, 2931, 2873, 1695, 1384, 1355, 1276, 1166, 1130, 1111, 1091, 1043, 1024, 923, 900, 844, 819, 748, 705, 698, $636 \mathrm{~cm}^{-1}$; HRMS (ESI-TOF) Calcd. for $\mathrm{C}_{42} \mathrm{H}_{22} \mathrm{~F}_{12} \mathrm{NO}_{3} \mathrm{~S}\left([\mathrm{M}-\mathrm{H}]^{-}\right)$: 848.1123, Found: 848.1128. 


\section{X-ray Structure of Chiral Ligand $(S, S)-4$}

$(S, S)$-4 was recrystallized from hexane.

The single crystal of $\mathbf{4}$ was mounted on a CryoLoop (Hampton Research Co. Ltd.). Data of X-ray diffraction were collected by a Rigaku RAXIS-RAPID Imaging Plate twodimensional area detector using graphite-monochromated MoK $\alpha$ radiation $(\lambda=0.71075$ $\AA$ ) to a maximum $2 \theta$ value of $55.0^{\circ}$. Crystallographic calculation was performed using CrystalStructure software package of the Rigaku Corporation and Molecular Structure Corporation. The crystal structure was solved by the direct method and refined by the full-matrix least squares using SIR-2002. All non-hydrogen atoms were refined anisotropically and hydrogen atoms were refined using the riding model. The crystallographic data are summarized in the following table.

\section{Experimental Details}

\begin{tabular}{|c|c|}
\hline empirical formula & $\mathrm{C}_{42} \mathrm{H}_{23} \mathrm{~F}_{12} \mathrm{NO}_{3} \mathrm{~S}$ \\
\hline formula weight & 849.69 \\
\hline crystal system & monoclinic \\
\hline space group & $\mathrm{P} 2_{1}(\# 4)$ \\
\hline$a, \AA$ & $16.029(4)$ \\
\hline$b, \AA$ & $13.029(3)$ \\
\hline$c, \AA$ & $17.866(6)$ \\
\hline$V, \AA^{3}$ & $3630.1(16)$ \\
\hline$Z$ & 4 \\
\hline$D_{\text {calc }}, \quad \mathrm{g} / \mathrm{cm}^{3}$ & 1.555 \\
\hline$T,{ }^{\circ} \mathrm{C}$ & -150.0 \\
\hline$\mu(\operatorname{MoK} \alpha), \mathrm{cm}^{-1}$ & 1.942 \\
\hline no. of reflns meased & 16618 \\
\hline no. of reflns obsd & 15200 \\
\hline no. of variable & 1122 \\
\hline$R 1$ & 0.0506 \\
\hline$R w$ & 0.1341 \\
\hline Goodness of fit & 1.326 \\
\hline Max shift/error in final cycle & 0.004 \\
\hline
\end{tabular}




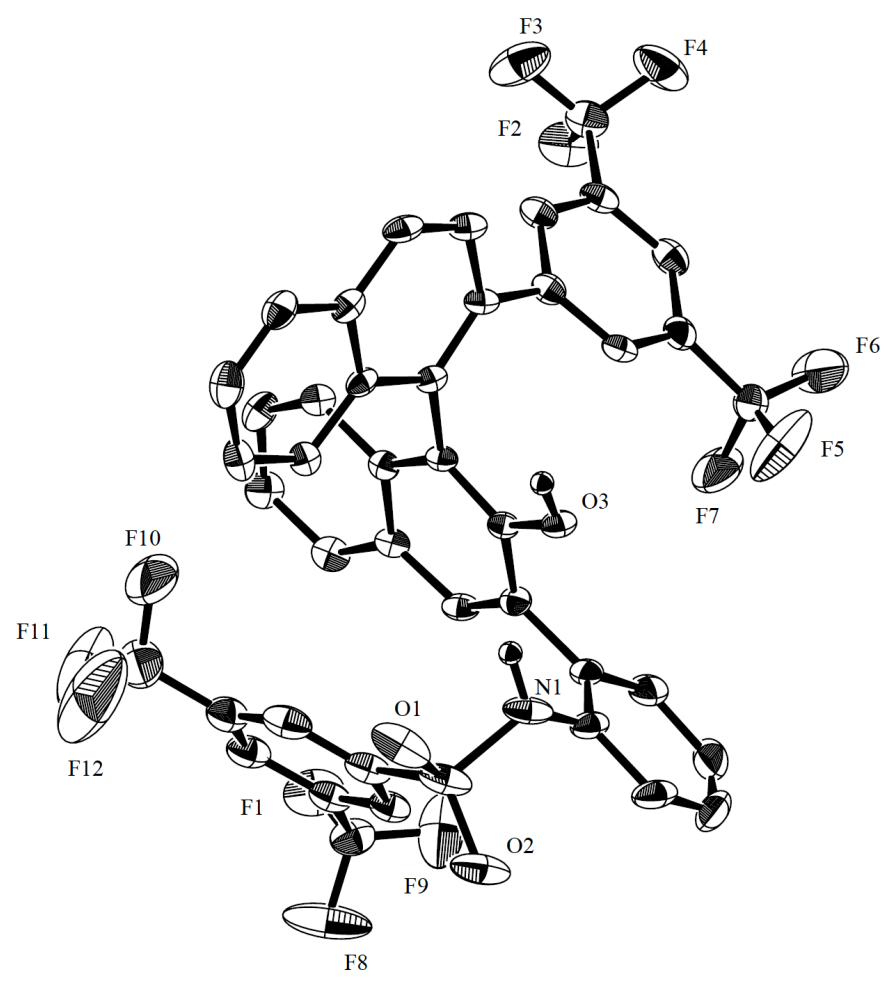

(a) ORTEP

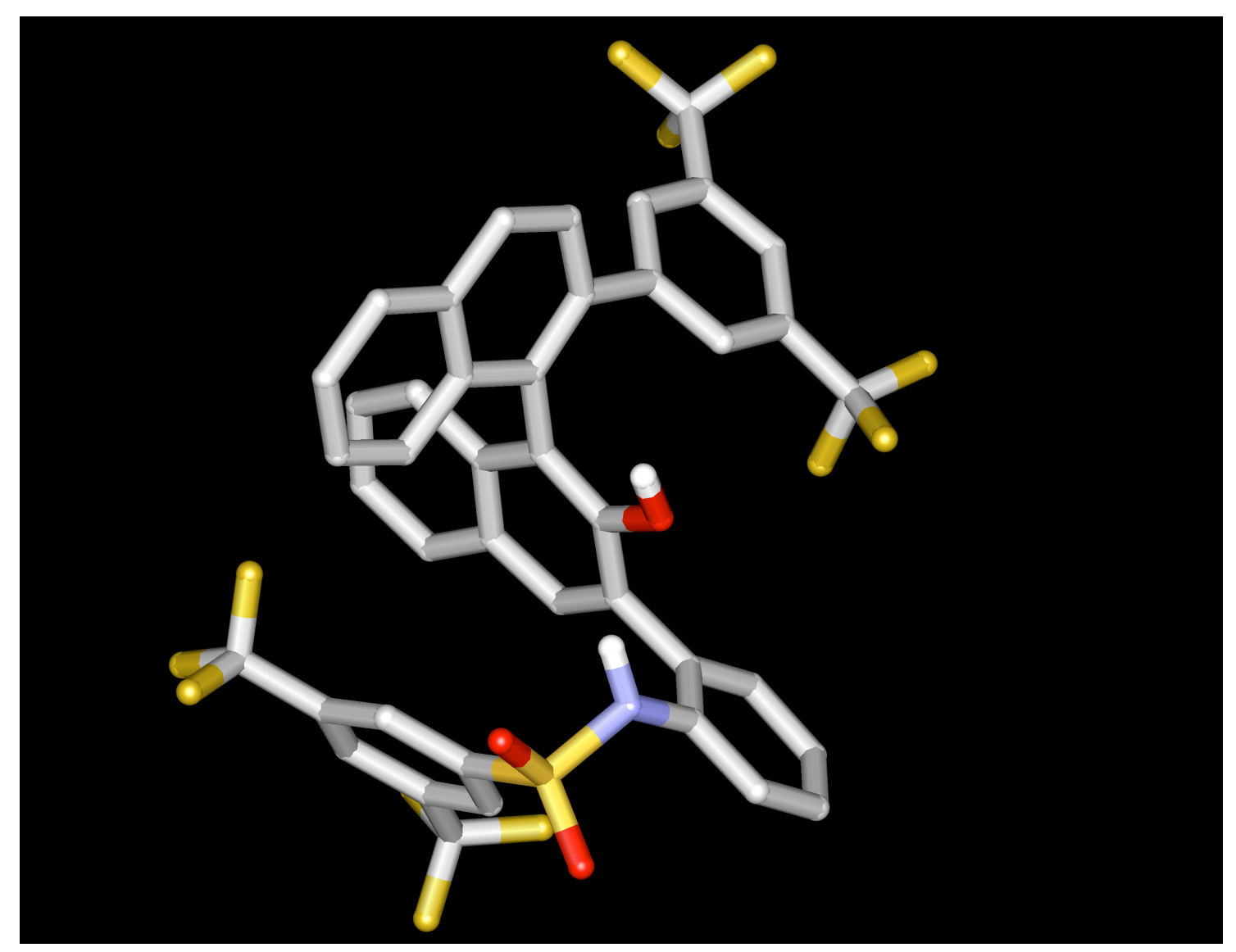

(b) Cylinder (F, S: Yellow, O: Red, N: Blue) 


\section{Characterization of Products}

$\mathrm{Ph}_{\mathrm{OSiEt}_{3}}^{\stackrel{\mathrm{Ph}}{\|}} \begin{aligned} & \left.\text { 1,4-Diphenyl-3-triethylsiloxy-2-butanone [2a }\left(\mathrm{R}^{2}=\mathrm{Et}\right)\right] \\ & \left.[\alpha]_{\mathrm{D}}{ }^{21}-58.7^{\circ}(c) 1.07, \mathrm{CHCl}_{3}, 86 \% \text { ee }\right) ;{ }^{1} \mathrm{H} \mathrm{NMR}\left(400 \mathrm{MHz}, \mathrm{CDCl}_{3}\right) \delta\end{aligned}$ 7.21-7.31 (6H, m, Ph-H), 7.16-7.18 (2H, m, Ph-H), 7.05-7.07 (2H, m, Ph-H), 4.36 (1H, dd, $J=7.6,4.4 \mathrm{~Hz}, \mathrm{SiOCH}), 3.77\left(1 \mathrm{H}, \mathrm{d}, J=17.2 \mathrm{~Hz}, \mathrm{PhCH}_{2} \mathrm{CO}\right), 3.66$, $(1 \mathrm{H}, \mathrm{d}, J=$ 17.2 Hz, $\left.\mathrm{PhCH}_{2} \mathrm{CO}\right), 2.93\left(1 \mathrm{H}, \mathrm{dd}, J=13.6,4.4 \mathrm{~Hz}, \mathrm{CHCH}_{2} \mathrm{Ph}\right), 2.83(1 \mathrm{H}, \mathrm{dd}, J=13.6$, $\left.7.6 \mathrm{~Hz}, \mathrm{CHCH}_{2} \mathrm{Ph}\right), 0.86\left(9 \mathrm{H}, \mathrm{t}, J=8.0 \mathrm{~Hz}, \mathrm{CH}_{3} \mathrm{CH}_{2} \mathrm{Si}\right), 0.41-0.52\left(6 \mathrm{H}, \mathrm{m}, \mathrm{CH}_{3} \mathrm{C}_{2} \mathrm{Si}\right)$; ${ }^{13} \mathrm{C}$ NMR $\left(100 \mathrm{MHz}, \mathrm{CDCl}_{3}\right) \delta 209.8,136.6,133.6,129.6,129.5,128.1,128.0,126.5$, 126.4, 79.4, 44.8, 41.5, 6.7, 4.6; IR (neat) 3030, 2953, 2910, 2322, 1718, 1496, 1454, 1417, 1238, 1095, 1004, 943, 773, 727, 696, $667 \mathrm{~cm}^{-1}$; HRMS (ESI-TOF) Calcd. for $\mathrm{C}_{22} \mathrm{H}_{31} \mathrm{O}_{2} \mathrm{Si}\left([\mathrm{M}+\mathrm{H}]^{+}\right)$: 355.2088, Found: 355.2090; HPLC condition: DAICEL Chiralcel $\mathrm{OJ}-\mathrm{H}$, hexane $/ i-\mathrm{PrOH}=99: 1$, flow rate $=0.5 \mathrm{~mL} / \mathrm{min}, \lambda=223 \mathrm{~nm}$, retention time: 16.6 $\min (R), 18.6 \min (S)$. Absolute configuration was established by comparison of optical rotation with known literature value ${ }^{2}$ after desilylation as described below.

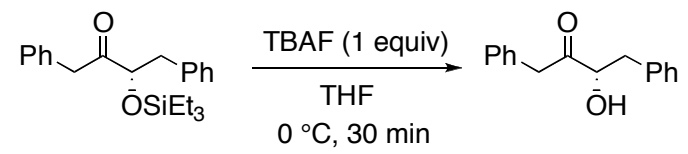

To a solution of $2 \mathbf{a}\left(\mathrm{R}^{2}=\mathrm{Et}\right)(177.3 \mathrm{mg}, 0.5 \mathrm{mmol})$ in THF $(2 \mathrm{~mL})$ was added a $1 \mathrm{M}$ THF solution of TBAF $(500 \mu \mathrm{L}, 0.5 \mathrm{mmol})$ at $0{ }^{\circ} \mathrm{C}$. After stirring for $30 \mathrm{~min}$, the mixture was poured into water and extracted with EtOAc. The organic extracts were washed with brine and dried over anhydrous $\mathrm{Na}_{2} \mathrm{SO}_{4}$. Removal of solvents and purification of the residual oil by short column chromatography on silica gel $($ EtOAc/hexane $=1 / 10$ as eluant $)$ afforded 1,4-diphenyl-3-hydroxy-2-butanone ${ }^{2}$ (120.2 $\mathrm{mg}, 0.5 \mathrm{mmol}$, quant).

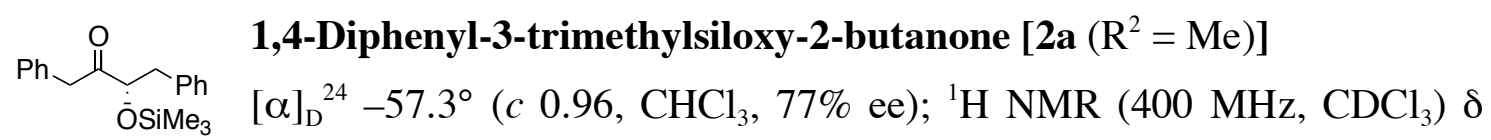
7.22-7.32 (6H, m, Ph-H), 7.16-7.20 (2H, m, Ph-H), 7.11-7.13 (2H, m, Ph-H), 4.28 (1H, $\mathrm{dd}, J=8.6,4.0 \mathrm{~Hz}, \mathrm{SiOCH}), 3.79\left(1 \mathrm{H}, \mathrm{d}, J=16.8 \mathrm{~Hz}, \mathrm{PhCH}_{2} \mathrm{CO}\right), 3.74,(1 \mathrm{H}, \mathrm{d}, J=$ $\left.16.8 \mathrm{~Hz}, \mathrm{PhCH}_{2} \mathrm{CO}\right), 2.95\left(1 \mathrm{H}, \mathrm{dd}, J=13.6,4.0 \mathrm{~Hz}, \mathrm{CHC}_{2} \mathrm{Ph}\right), 2.76(1 \mathrm{H}, \mathrm{dd}, J=13.6$, $\left.8.6 \mathrm{~Hz}, \mathrm{CHCH}_{2} \mathrm{Ph}\right),-0.10\left(9 \mathrm{H}, \mathrm{s}, \mathrm{Me}_{3} \mathrm{Si}\right) ;{ }^{13} \mathrm{C} \mathrm{NMR}\left(100 \mathrm{MHz}, \mathrm{CDCl}_{3}\right) \delta 210.1,137.3$, 134.1, 130.0, 129.9, 128.6, 128.4, 127.0, 126.8, 79.8, 45.1, 41.6, 0.0; IR (neat) 2954, 1720, 1496, 1454, 1251, 1097, 945, 902, 840, 748, $698 \mathrm{~cm}^{-1}$; HRMS (ESI-TOF) Calcd. for $\mathrm{C}_{19} \mathrm{H}_{24} \mathrm{NaO}_{2} \mathrm{Si}\left([\mathrm{M}+\mathrm{Na}]^{+}\right)$: 335.1449, Found: 335.1435; HPLC condition: DAICEL Chiralpak AD-H, hexane $/ i-\mathrm{PrOH}=99: 1$, flow rate $=0.5 \mathrm{~mL} / \mathrm{min}, \lambda=223 \mathrm{~nm}$, retention time: $9.7 \min (S), 11.1 \min (R)$. 


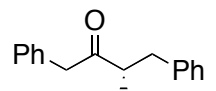

1,4-Diphenyl-3-tert-butyldimethylsiloxy-2-butanone $\left[2 \mathrm{a} \quad\left(\mathrm{R}_{3}^{2}=t\right.\right.$ $\left.\left.\overline{\mathrm{OSiMe}}_{2} \mathrm{Bu}^{t} \quad \mathrm{BuMe}_{2}\right)\right]$

$[\alpha]_{\mathrm{D}}{ }^{24}-41.2^{\circ}\left(c 1.01, \mathrm{CHCl}_{3}, 80 \%\right.$ ee $) ;{ }^{1} \mathrm{H} \mathrm{NMR}\left(400 \mathrm{MHz}, \mathrm{CDCl}_{3}\right) \delta 7.21-7.33(6 \mathrm{H}, \mathrm{m}$, Ph-H), 7.17-7.19 (2H, m, Ph-H), 7.07-7.09 (2H, m, Ph-H), 4.32 (1H, dd, J = 8.0, 4.0 Hz, $\mathrm{SiOCH}), 3.79\left(1 \mathrm{H}, \mathrm{d}, J=17.2 \mathrm{~Hz}, \mathrm{PhCH}_{2} \mathrm{CO}\right), 3.72\left(1 \mathrm{H}, \mathrm{d}, J=17.2 \mathrm{~Hz}, \mathrm{PhCH}_{2} \mathrm{CO}\right)$, $2.95\left(1 \mathrm{H}, \mathrm{dd}, J=13.6,4.0 \mathrm{~Hz}, \mathrm{CHC}_{2} \mathrm{Ph}\right), 2.76\left(1 \mathrm{H}, \mathrm{dd}, J=13.6,8.0 \mathrm{~Hz}, \mathrm{CHC}_{2} \mathrm{Ph}\right)$, 0.88 (9H, s, $t$-BuSi), -0.07 (3H, s, MeSi), -0.25 (3H, s, MeSi); ${ }^{13} \mathrm{C}$ NMR (100 MHz, $\left.\mathrm{CDCl}_{3}\right) \delta 210.3,136.8,133.8,129.9,129.7,128.3,128.2,126.7,126.6,79.7,44.6,41.4$, 25.7, 18.0, -5.2, -5.5; IR (neat) 3030, 2953, 2927, 2856, 1722, 1496, 1454, 1253, 1093, 943, 835, 812, 777, 752, $698 \mathrm{~cm}^{-1}$; HRMS (ESI-TOF) Calcd. for $\mathrm{C}_{22} \mathrm{H}_{30} \mathrm{NaO}_{2} \mathrm{Si}$ $\left([\mathrm{M}+\mathrm{Na}]^{+}\right)$: 377.1918, Found: 377.1915; HPLC condition: DAICEL Chiralcel OJ-H, hexane $/ i-\mathrm{PrOH}=99: 1$, flow rate $=0.5 \mathrm{~mL} / \mathrm{min}, \lambda=223 \mathrm{~nm}$, retention time: $14.3 \mathrm{~min}$ $(R), 18.2 \min (S)$.

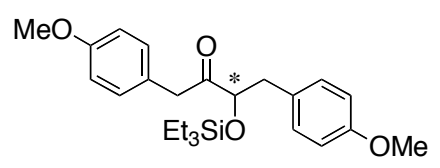

1,4-Bis(4-methoxyphenyl)-3-triethylsiloxy-2-butanone (2b)

$[\alpha]_{\mathrm{D}}{ }^{29}-197.9^{\circ}\left(c 1.51, \mathrm{CHCl}_{3}, 85 \%\right.$ ee $) ;{ }^{1} \mathrm{H} \mathrm{NMR}(400 \mathrm{MHz}$, $\left.\mathrm{CDCl}_{3}\right) \delta 7.08(2 \mathrm{H}, \mathrm{d}, J=8.4 \mathrm{~Hz}, \mathrm{Ph}-\mathrm{H}), 6.97(2 \mathrm{H}, \mathrm{d}, J=8.4 \mathrm{~Hz}, \mathrm{Ph}-\mathrm{H}), 6.79-6.84(4 \mathrm{H}$, m, Ph-H), 4.32 (1H, dd, J = 7.2, $4.8 \mathrm{~Hz}, \mathrm{SiOCH}), 3.79$ (3H, s, MeOPh), 3.78 (3H, s, $\mathrm{MeOPh}), 3.69$ (1H, d, $\left.J=17.2 \mathrm{~Hz}, \mathrm{PhCH}_{2} \mathrm{CO}\right), 3.58\left(1 \mathrm{H}, \mathrm{d}, J=17.2 \mathrm{~Hz}, \mathrm{PhCH}_{2} \mathrm{CO}\right)$, $2.87\left(1 \mathrm{H}, \mathrm{dd}, J=13.6,4.8 \mathrm{~Hz}, \mathrm{CHCH}_{2} \mathrm{Ph}\right), 2.79\left(1 \mathrm{H}, \mathrm{dd}, J=13.6,7.2 \mathrm{~Hz}, \mathrm{CHCH}_{2} \mathrm{Ph}\right)$, $0.88\left(9 \mathrm{H}, \mathrm{t}, J=8.0 \mathrm{~Hz}, \mathrm{CH}_{3} \mathrm{CH}_{2} \mathrm{Si}\right), 0.44-0.56\left(6 \mathrm{H}, \mathrm{m}, \mathrm{CH}_{3} \mathrm{CH}_{2} \mathrm{Si}\right) ;{ }^{13} \mathrm{C}$ NMR $(100 \mathrm{MHz}$, $\left.\mathrm{CDCl}_{3}\right) \delta 210.3,158.2,130.5,130.5,128.6,125.6,113.6,113.4,79.4,55.1,55.0,43.9$, 40.7, 6.7, 4.6; IR (neat) 2953, 2910, 2875, 2835, 1718, 1612, 1583, 1510, 1463, 1440, 1300, 1246, 1176, 1109, 1089, 1035, 1004, 945, 819, 802, 765, 740, 729, $678 \mathrm{~cm}^{-1}$; HRMS (ESI-TOF) Calcd. for $\mathrm{C}_{30} \mathrm{H}_{34} \mathrm{NaO}_{2} \mathrm{Si}\left([\mathrm{M}+\mathrm{Na}]^{+}\right)$: 437.2118, Found: 437.2115; HPLC condition: DAICEL Chiralcel OD-H, hexane $/ i-\mathrm{PrOH}=99: 1$, flow rate $=0.5$ $\mathrm{mL} / \mathrm{min}, \lambda=223 \mathrm{~nm}$, retention time: $28.5 \mathrm{~min}$ (major), $36.9 \mathrm{~min}$ (minor).

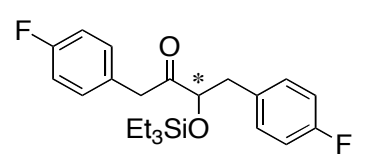

\section{1,4-Bis(4-fluorophenyl)-3-triethylsiloxy-2-butanone (2c)}

$[\alpha]_{\mathrm{D}}{ }^{29}-66.4^{\circ}$ (c 1.11, $\mathrm{CHCl}_{3}, 90 \%$ ee); ${ }^{1} \mathrm{H} \mathrm{NMR}(400 \mathrm{MHz}$, $\left.\mathrm{CDCl}_{3}\right) \delta$ 7.10-7.14 (2H, m, Ph-H), 6.94-7.01 (6H, m, Ph-H), $4.33(1 \mathrm{H}, \mathrm{dd}, J=7.2,4.6 \mathrm{~Hz}, \mathrm{SiOCH}), 3.73\left(1 \mathrm{H}, \mathrm{d}, J=17.2 \mathrm{~Hz}, \mathrm{PhCH}_{2} \mathrm{CO}\right), 3.61(1 \mathrm{H}$, $\left.\mathrm{d}, J=17.2 \mathrm{~Hz}, \mathrm{PhCH}_{2} \mathrm{CO}\right), 2.88\left(1 \mathrm{H}, \mathrm{dd}, J=14.0,4.6 \mathrm{~Hz}, \mathrm{CHCH}_{2} \mathrm{Ph}\right), 2.83(1 \mathrm{H}, \mathrm{dd}, J=$ $\left.14.0,7.2 \mathrm{~Hz}, \mathrm{CHC}_{2} \mathrm{Ph}\right), 0.89\left(9 \mathrm{H}, \mathrm{t}, J=8.0 \mathrm{~Hz}, \mathrm{C}_{3} \mathrm{CH}_{2} \mathrm{Si}\right), 0.45-0.55(6 \mathrm{H}, \mathrm{m}$, $\left.\mathrm{CH}_{3} \mathrm{CH}_{2} \mathrm{Si}\right) ;{ }^{13} \mathrm{C} \mathrm{NMR}\left(100 \mathrm{MHz}, \mathrm{CDCl}_{3}\right) \delta 209.9,161.7\left(\mathrm{~d}, J_{\mathrm{C}-\mathrm{F}}=246 \mathrm{~Hz}\right), 161.6\left(\mathrm{~d}, J_{\mathrm{C}-}\right.$ $\left.\mathrm{F}_{\mathrm{F}}=246 \mathrm{~Hz}\right), 132.2\left(\mathrm{~d}, J_{\mathrm{C}-\mathrm{F}}=3.3 \mathrm{~Hz}\right), 131.2\left(\mathrm{~d}, J_{\mathrm{C}-\mathrm{F}}=8.3 \mathrm{~Hz}\right), 131.0\left(\mathrm{~d}, J_{\mathrm{C}-\mathrm{F}}=8.2 \mathrm{~Hz}\right)$, $129.2\left(\mathrm{~d}, J_{\mathrm{C}-\mathrm{F}}=3.3 \mathrm{~Hz}\right), 115.1\left(\mathrm{~d}, J_{\mathrm{C}-\mathrm{F}}=21.4 \mathrm{~Hz}\right), 114.9\left(\mathrm{~d}, J_{\mathrm{C}-\mathrm{F}}=20.6 \mathrm{~Hz}\right), 79.3,43.8$, 
40.7, 6.7, 4.6; IR (neat) 2954, 2912, 2877, 1720, 1602, 1508, 1458, 1415, 1340, 1220, 1157, 1099, 1087, 1004, 974, 945, 823, 804, 769, 740, 729, $678 \mathrm{~cm}^{-1}$; HRMS (ESI-TOF) Calcd. for $\mathrm{C}_{22} \mathrm{H}_{29} \mathrm{~F}_{2} \mathrm{O}_{2} \mathrm{Si}\left([\mathrm{M}+\mathrm{H}]^{+}\right)$: 391.1899, Found: 391.1897; HPLC condition: DAICEL Chiralpak AD-H, hexane $/ i-\mathrm{PrOH}=99: 1$, flow rate $=0.5 \mathrm{~mL} / \mathrm{min}, \lambda=223 \mathrm{~nm}$, retention time: 14.4 min (major), 16.1 min (minor).

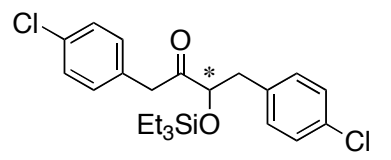

\section{1,4-Bis(4-chlorophenyl)-3-triethylsiloxy-2-butanone (2d)}

$[\alpha]_{\mathrm{D}}{ }^{26}-67.9^{\circ}$ (c 1.03, $\mathrm{CHCl}_{3}, 86 \%$ ee); ${ }^{1} \mathrm{H}$ NMR $(400 \mathrm{MHz}$, $\left.\mathrm{CDCl}_{3}\right) \delta$ 7.22-7.25 (4H, m, Ph-H), $7.08(2 \mathrm{H}, \mathrm{d}, J=8.8 \mathrm{~Hz}, \mathrm{Ph}-$ H), 6.94 (2H, d, J = 8.0 Hz, Ph-H), 4.34 (1H, dd, J = 6.8, 4.8 Hz, SiOCH), $3.71(1 \mathrm{H}, \mathrm{d}$, $\left.J=17.2 \mathrm{~Hz}, \mathrm{PhCH}_{2} \mathrm{CO}\right), 3.61\left(1 \mathrm{H}, \mathrm{d}, J=17.2 \mathrm{~Hz}, \mathrm{PhCH}_{2} \mathrm{CO}\right), 2.88(1 \mathrm{H}, \mathrm{dd}, J=13.6$, $\left.4.8 \mathrm{~Hz}, \mathrm{CHC}_{2} \mathrm{Ph}\right), 2.83\left(1 \mathrm{H}, \mathrm{dd}, J=13.6,6.8 \mathrm{~Hz}, \mathrm{CHC}_{2} \mathrm{Ph}\right), 0.90(9 \mathrm{H}, \mathrm{t}, J=8.0 \mathrm{~Hz}$, $\left.\mathrm{C}_{3} \mathrm{CH}_{2} \mathrm{Si}\right), 0.46-0.57\left(6 \mathrm{H}, \mathrm{m}, \mathrm{CH}_{3} \underline{\mathrm{C}}_{2} \mathrm{Si}\right) ;{ }^{13} \mathrm{C} \mathrm{NMR}\left(100 \mathrm{MHz}, \mathrm{CDCl}_{3}\right) \delta 209.5,134.9$, 132.6, 132.5, 131.9, 131.0, 128.3, 128.2, 79.1, 44.0, 40.9, 6.8, 4.7; IR (neat) 2954, 2910, 2875, 1720, 1490, 1458, 1408, 1338, 1240, 1089, 1016, 974, 947, 910, 842, 792, 767, 729, $677 \mathrm{~cm}^{-1}$; HRMS (ESI-TOF) Calcd. for $\mathrm{C}_{22} \mathrm{H}_{29} \mathrm{Cl}_{2} \mathrm{O}_{2} \mathrm{Si}\left([\mathrm{M}+\mathrm{H}]^{+}\right)$: 423.1308, Found: 423.1299; HPLC condition: DAICEL Chiralpak AD-H, hexane $/ i-\mathrm{PrOH}=99: 1$, flow rate $=0.5 \mathrm{~mL} / \mathrm{min}, \lambda=223 \mathrm{~nm}$, retention time: $22.1 \mathrm{~min}$ (major), $23.8 \mathrm{~min}$ (minor).

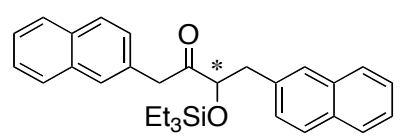

\section{1,4-Di(2-naphthyl)-3-triethylsiloxy-2-butanone (2e)}

$[\alpha]_{\mathrm{D}}^{23}-81.0^{\circ}$ (c 1.16, $\mathrm{CHCl}_{3}, 85 \%$ ee); ${ }^{1} \mathrm{H}$ NMR (400 MHz, $\left.\mathrm{CDCl}_{3}\right) \delta$ 7.62-7.80 (5H, m, Ar-H), $7.57(1 \mathrm{H}, \mathrm{s}, \mathrm{Ar}-\mathrm{H}), 7.40-7.46(4 \mathrm{H}, \mathrm{m}, \mathrm{Ar}-\mathrm{H}), 7.36$ $(1 \mathrm{H}, \mathrm{dd}, J=8.4,1.6 \mathrm{~Hz}, \mathrm{Ar}-\mathrm{H}), 7.34(1 \mathrm{H}, \mathrm{s}, \mathrm{Ar}-\mathrm{H}), 7.12(1 \mathrm{H}, \mathrm{dd}, J=8.4,1.6 \mathrm{~Hz}, \mathrm{Ar}-\mathrm{H})$, $4.52(1 \mathrm{H}, \mathrm{dd}, J=6.8,5.0 \mathrm{~Hz}, \mathrm{SiOCH}), 3.87\left(1 \mathrm{H}, \mathrm{d}, J=16.8 \mathrm{~Hz}, \mathrm{ArCH}_{2} \mathrm{CO}\right), 3.79(1 \mathrm{H}$, $\left.\mathrm{d}, J=16.8 \mathrm{~Hz}, \mathrm{ArCH}_{2} \mathrm{CO}\right), 3.12\left(1 \mathrm{H}, \mathrm{dd}, J=13.6,5.0 \mathrm{~Hz}, \mathrm{CHC}_{2} \mathrm{Ar}\right), 3.07(1 \mathrm{H}, \mathrm{dd}, J=$ 13.6, $\left.6.8 \mathrm{~Hz}, \mathrm{CHC}_{2} \mathrm{Ar}\right), 0.89\left(9 \mathrm{H}, \mathrm{t}, J=8.0 \mathrm{~Hz}, \mathrm{C}_{3} \mathrm{CH}_{2} \mathrm{Si}\right), 0.48-0.55(6 \mathrm{H}, \mathrm{m}$, $\left.\mathrm{CH}_{3} \mathrm{CH}_{2} \mathrm{Si}\right) ;{ }^{13} \mathrm{C}$ NMR $\left(100 \mathrm{MHz}, \mathrm{CDCl}_{3}\right) \delta 210.2,134.0,133.2,133.2,132.2,132.1$, 131.1, 128.4, 128.2, 128.0, 127.8, 127.7, 127.6, 127.4, 127.4, 127.4, 125.8, 125.7, 125.4, 125.3, 79.4, 45.2, 41.8, 6.8, 4.7; IR (neat) 3053, 2953, 2908, 2875, 2357, 2313, 1718, 1508, 1456, 1417, 1338, 1238, 1093, 1016, 962, 854, 808, $742 \mathrm{~cm}^{-1}$; HRMS (ESI-TOF) Calcd. for $\mathrm{C}_{30} \mathrm{H}_{34} \mathrm{NaO}_{2} \mathrm{Si}\left([\mathrm{M}+\mathrm{Na}]^{+}\right)$: 477.2231, Found: 477.2223; HPLC condition: DAICEL Chiralpak AS-H, hexane $/ i-\mathrm{PrOH}=99: 1$, flow rate $=0.3 \mathrm{~mL} / \mathrm{min}, \lambda=223 \mathrm{~nm}$, retention time: $23.7 \mathrm{~min}$ (major), $27.1 \mathrm{~min}$ (minor).

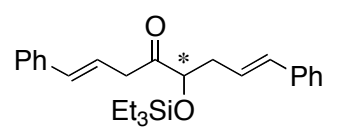

\section{1,8-Diphenyl-5-triethylsiloxy-1,7-octadien-4-one (2f)}

$[\alpha]_{\mathrm{D}}^{27}-32.0^{\circ}$ (c 1.09, $\mathrm{CHCl}_{3}, 83 \%$ ee); ${ }^{1} \mathrm{H} \mathrm{NMR}(400 \mathrm{MHz}$, $\left.\mathrm{CDCl}_{3}\right) \delta$ 7.26-7.33 (8H, m, Ph-H), 7.18-7.22 (2H, m, Ph-H), $6.94(2 \mathrm{H}, \mathrm{d}, J=8.0 \mathrm{~Hz}$, $\mathrm{Ph}-\mathrm{H}), 6.42(1 \mathrm{H}, \mathrm{d}, J=16.0 \mathrm{~Hz}, \mathrm{PhCH}), 6.40(1 \mathrm{H}, \mathrm{d}, J=16.0 \mathrm{~Hz}, \mathrm{PhCH}), 6.31(1 \mathrm{H}, \mathrm{dt}$, $J=16.0,6.4 \mathrm{~Hz}, \mathrm{PhCH}=\mathrm{C} \underline{\mathrm{H}}), 6.19(1 \mathrm{H}, \mathrm{dt}, J=16.0,6.4 \mathrm{~Hz}, \mathrm{PhCH}=\mathrm{C} \underline{\mathrm{H}}), 4.25(1 \mathrm{H}, \mathrm{t}, J$ 
$=6.4 \mathrm{~Hz}, \mathrm{SiOCH}), 3.51\left(2 \mathrm{H}, \mathrm{d}, J=6.4 \mathrm{~Hz}, \mathrm{CHCH}_{2} \mathrm{CO}\right), 2.57(2 \mathrm{H}, \mathrm{t}, J=6.4 \mathrm{~Hz}$, $\left.\mathrm{SiOCHCH} \underline{H}_{2} \mathrm{CH}\right), 0.97\left(9 \mathrm{H}, \mathrm{t}, J=8.0 \mathrm{~Hz}, \mathrm{CH}_{3} \mathrm{CH}_{2} \mathrm{Si}\right), 0.64(6 \mathrm{H}, \mathrm{q}, J=8.0 \mathrm{~Hz}$, $\left.\mathrm{CH}_{3} \mathrm{C}_{2} \mathrm{Si}\right) ;{ }^{13} \mathrm{C}$ NMR (100 MHz, $\left.\mathrm{CDCl}_{3}\right) \delta 211.3,137.1,136.9,133.4,133.3,128.4$, 128.4, 127.3, 127.2, 126.1, 126.1, 124.4, 121.9, 78.4, 41.8, 38.9, 6.7, 4.7; IR (neat) 3026, 2954, 2910, 2875, 1716, 1598, 1494, 1448, 1240, 1099, 1004, 964, 831, 736, $692 \mathrm{~cm}^{-1}$; HRMS (ESI-TOF) Calcd. for $\mathrm{C}_{26} \mathrm{H}_{34} \mathrm{NaO}_{2} \mathrm{Si}\left([\mathrm{M}+\mathrm{Na}]^{+}\right)$: 429.2231, Found: 429.2229; HPLC condition: DAICEL Chiralcel OJ-H, hexane $/ i-\mathrm{PrOH}=99: 1$, flow rate $=0.5$ $\mathrm{mL} / \mathrm{min}, \lambda=254 \mathrm{~nm}$, retention time: $34.7 \mathrm{~min}$ (minor), $40.7 \mathrm{~min}$ (major).

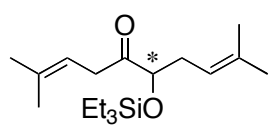

2,9-Dimethyl-6-triethylsiloxy-2,8-decadien-5-one (2g)

$[\alpha]_{\mathrm{D}}{ }^{27}-6.5^{\circ}\left(c 0.98, \mathrm{CHCl}_{3}, 80 \%\right.$ ee); ${ }^{1} \mathrm{H} \mathrm{NMR}\left(400 \mathrm{MHz}, \mathrm{CDCl}_{3}\right) \delta$ 5.27-5.31 (1H, m, C=CH), 5.10-5.14 $(1 \mathrm{H}, \mathrm{m}, \mathrm{C}=\mathrm{CH}), 4.07(1 \mathrm{H}, \mathrm{dd}, J=6.6,5.8 \mathrm{~Hz}$, $\mathrm{SiOCH}), 3.28\left(2 \mathrm{H}, \mathrm{d}, J=7.2 \mathrm{~Hz}, \mathrm{CHCH}_{2} \mathrm{CO}\right), 2.26-2.40\left(2 \mathrm{H}, \mathrm{m}, \mathrm{SiCHCH}_{2} \mathrm{CH}\right), 1.74$ $\left(3 \mathrm{H}, \mathrm{s}, \mathrm{Me}_{2} \mathrm{C}=\right), 1.69\left(3 \mathrm{H}, \mathrm{s}, \mathrm{Me}_{2} \mathrm{C}=\right), 1.60\left(3 \mathrm{H}, \mathrm{s}, \mathrm{Me}_{2} \mathrm{C}=\right), 1.58\left(3 \mathrm{H}, \mathrm{s}, \mathrm{Me}_{2} \mathrm{C}=\right), 0.95$ $\left(9 \mathrm{H}, \mathrm{t}, J=8.0 \mathrm{~Hz}, \mathrm{C}_{3} \mathrm{CH}_{2} \mathrm{Si}\right), 0.60\left(6 \mathrm{H}, \mathrm{q}, J=8.0 \mathrm{~Hz}, \mathrm{CH}_{3} \underline{\mathrm{C}}_{2} \mathrm{Si}\right) ;{ }^{13} \mathrm{C} \mathrm{NMR}(100$ $\left.\mathrm{MHz}, \mathrm{CDCl}_{3}\right) \delta 211.9,135.1,134.5,118.7,115.8,78.5,37.1,34.0,25.7,25.7,18.0,17.9$, 6.7, 4.7; IR (neat) 2956, 2912, 2875, 1737, 1456, 1377, 1238, 1143, 1091, 1010, 839, 740, $729 \mathrm{~cm}^{-1}$; HRMS (ESI-TOF) Calcd. for $\mathrm{C}_{18} \mathrm{H}_{34} \mathrm{NaO}_{2} \mathrm{Si}\left([\mathrm{M}+\mathrm{Na}]^{+}\right)$: 333.2231, Found: 333.2224. Enantiomeric excess was determined by HPLC analysis after desilylation (see the above mentioned procedure).

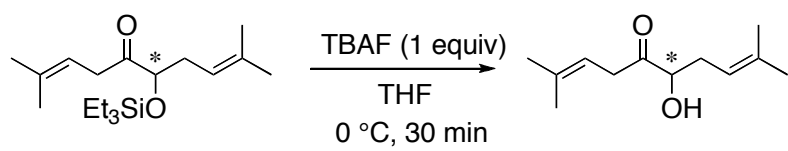

HPLC condition: See also ref. 2.

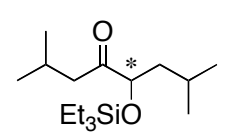

\section{2,7-Dimethyl-5-triethylsiloxy-4-octanone (2h)}

$[\alpha]_{\mathrm{D}}^{27}-13.3^{\circ}$ (c 1.00, $\mathrm{CHCl}_{3}, 74 \%$ ee); ${ }^{1} \mathrm{H}$ NMR (400 MHz, $\left.\mathrm{CDCl}_{3}\right) \delta$ 4.04 (1H, dd, $J=8.6,5.4 \mathrm{~Hz}, \mathrm{SiOCH}), 2.41\left(2 \mathrm{H}, \mathrm{d}, J=7.2 \mathrm{~Hz}, \mathrm{CH}_{2} \mathrm{CO}\right), 2.12-2.19(1 \mathrm{H}$, $\left.\mathrm{m}, \mathrm{Me}_{2} \mathrm{C} \underline{\mathrm{H}}\right), 1.67-1.74\left(1 \mathrm{H}, \mathrm{m}, \mathrm{Me}_{2} \mathrm{C} \underline{\mathrm{H}}\right), 1.26-1.50\left(2 \mathrm{H}, \mathrm{m}, \mathrm{SiOCHC}_{2}\right), 0.87-0.98(12 \mathrm{H}$, m, $\left.\underline{\mathrm{Me}}_{2} \mathrm{CH}\right), 0.93\left(9 \mathrm{H}, \mathrm{t}, J=8.0 \mathrm{~Hz}, \underline{\mathrm{C}}_{3} \mathrm{CH}_{2} \mathrm{Si}\right), 0.60\left(6 \mathrm{H}, \mathrm{q}, J=8.0 \mathrm{~Hz}, \mathrm{CH}_{3} \mathrm{C}_{2} \mathrm{Si}\right)$; ${ }^{13} \mathrm{C} \mathrm{NMR}\left(100 \mathrm{MHz}, \mathrm{CDCl}_{3}\right) \delta$ 213.0, 77.4, 45.6, 43.7, 23.9, 23.4, 23.1, 22.6, 22.5, 22.0, 6.7, 4.7; IR (neat) 2956, 2937, 2912, 2875, 1714, 1467, 1367, 1240, 1095, 1004, 904, 742, $729 \mathrm{~cm}^{-1}$; HRMS (ESI-TOF) Calcd. for $\mathrm{C}_{16} \mathrm{H}_{34} \mathrm{NaO}_{2} \mathrm{Si}\left([\mathrm{M}+\mathrm{Na}]^{+}\right)$: 309.2231, Found: 309.2218. Enantiomeric excess was determined by HPLC analysis after desilylation (see the above mentioned procedure) and subsequent reprotection as 3,5dinitrobenzoate. ${ }^{3}$ 

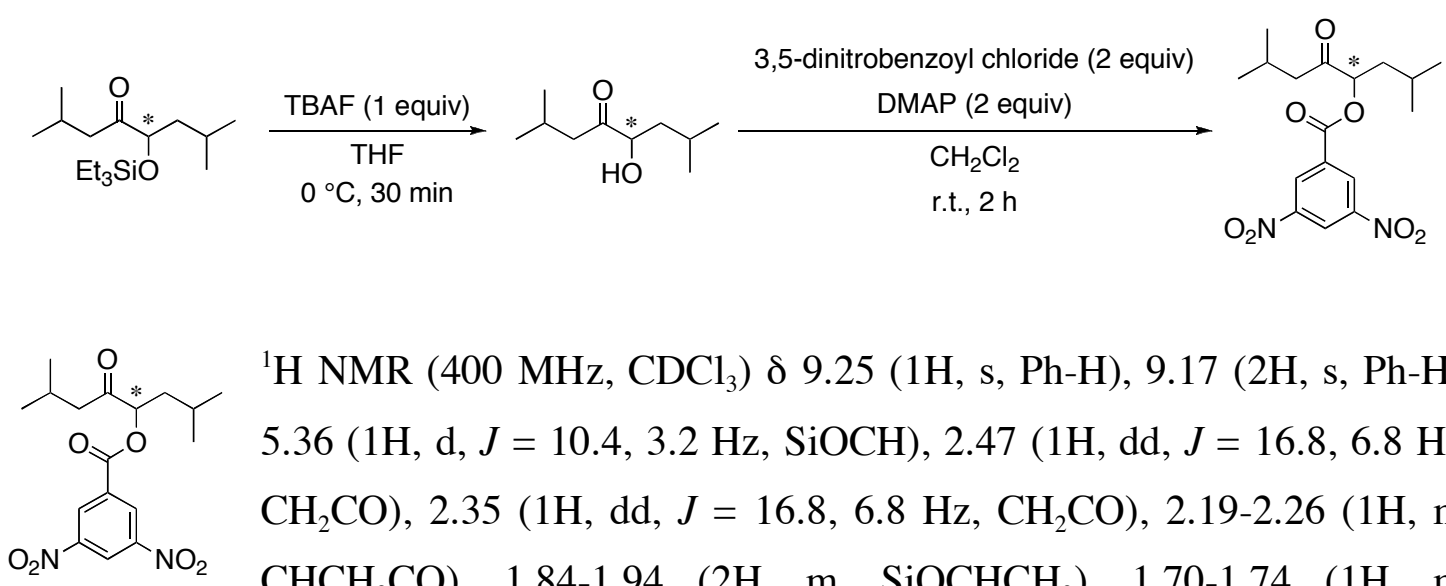

${ }^{1} \mathrm{H}$ NMR (400 MHz, CDCl $)$ ) 9.25 (1H, s, Ph-H), 9.17 (2H, s, Ph-H), $5.36(1 \mathrm{H}, \mathrm{d}, J=10.4,3.2 \mathrm{~Hz}, \mathrm{SiOCH}), 2.47(1 \mathrm{H}, \mathrm{dd}, J=16.8,6.8 \mathrm{~Hz}$, $\left.\mathrm{CH}_{2} \mathrm{CO}\right), 2.35\left(1 \mathrm{H}, \mathrm{dd}, J=16.8,6.8 \mathrm{~Hz}, \mathrm{CH}_{2} \mathrm{CO}\right), 2.19-2.26(1 \mathrm{H}, \mathrm{m}$,

$\left.\mathrm{CHCH}_{2} \mathrm{CO}\right), 1.84-1.94\left(2 \mathrm{H}, \mathrm{m}, \mathrm{SiOCHC} \underline{H}_{2}\right), 1.70-1.74 \quad(1 \mathrm{H}, \mathrm{m}$, $\left.\mathrm{SiOCHCH}_{2} \mathrm{C} \underline{\mathrm{H}}\right), 1.05$ (3H, d, $\left.J=6.8 \mathrm{~Hz}, \underline{\mathrm{Me}}_{2} \mathrm{CH}\right), 1.02\left(3 \mathrm{H}, \mathrm{d}, J=6.8 \mathrm{~Hz}, \underline{\mathrm{Me}}_{2} \mathrm{CH}\right)$, $0.98\left(3 \mathrm{H}, \mathrm{d}, J=6.8 \mathrm{~Hz}, \mathrm{Me}_{2} \mathrm{CH}\right), 0.96\left(3 \mathrm{H}, \mathrm{d}, J=6.8 \mathrm{~Hz}, \mathrm{Me}_{2} \mathrm{CH}\right) ;{ }^{13} \mathrm{C} \mathrm{NMR}(100 \mathrm{MHz}$, $\left.\mathrm{CDCl}_{3}\right) \delta 204.9,162.1,148.6,133.2,129.5,122.5,79.4,47.3,38.4,25.0,24.2,23.2$, 22.4, 22.4, 21.2; HPLC condition: DAICEL Chiralpak AS-H, hexane $/ i-\mathrm{PrOH}=97: 3$, flow rate $=0.5 \mathrm{~mL} / \mathrm{min}, \lambda=254 \mathrm{~nm}$, retention time: $16.8 \mathrm{~min}$ (minor), $19.6 \mathrm{~min}$ (major).

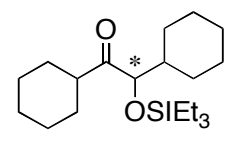

\section{1,2-Dicyclohexyl-2-triethylsiloxyethanone (2i)}

$[\alpha]_{\mathrm{D}}{ }^{25}-5.9^{\circ}$ (c 1.10, $\mathrm{CHCl}_{3}, 74 \%$ ee); ${ }^{1} \mathrm{H}$ NMR (400 MHz, $\left.\mathrm{CDCl}_{3}\right) \delta 3.89$ $(1 \mathrm{H}, \mathrm{d}, J=5.2 \mathrm{~Hz}, \mathrm{SiOCH}), 2.73-2.79(1 \mathrm{H}, \mathrm{m}, \mathrm{CHCO}), 1.52-1.79(11 \mathrm{H}, \mathrm{m},-\mathrm{CH}-), 1.06-$ $1.43(10 \mathrm{H}, \mathrm{m},-\mathrm{CH}-), 0.95\left(9 \mathrm{H}, \mathrm{t}, J=8.0 \mathrm{~Hz}, \mathrm{CH}_{3} \mathrm{CH}_{2} \mathrm{Si}\right), 0.59(6 \mathrm{H}, \mathrm{q}, J=8.0 \mathrm{~Hz}$,

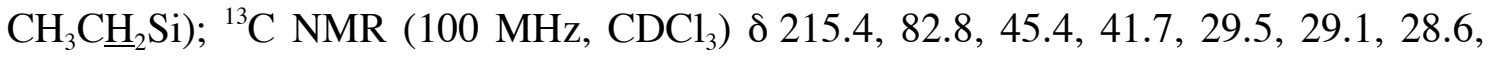
27.7, 26.2, 26.1, 26.1, 25.8, 25.7, 25.5, 6.8, 4.8; IR (neat) 2929, 2854, 1716, 1450, 1238, 1141, 1004, 835, $740 \mathrm{~cm}^{-1}$; HRMS (ESI-TOF) Calcd. for $\mathrm{C}_{20} \mathrm{H}_{38} \mathrm{NaO}_{2} \mathrm{Si}\left([\mathrm{M}+\mathrm{Na}]^{+}\right)$: 361.2533, Found: 361.2546. Enantiomeric excess was determined in a similar manner as described for $\mathbf{2 h}$.

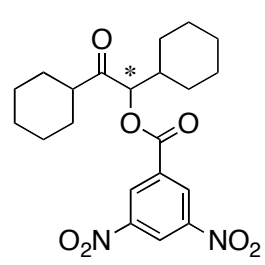

${ }^{1} \mathrm{H}$ NMR (400 MHz, $\left.\mathrm{CDCl}_{3}\right) \delta 9.25$ (1H, s, Ph-H), 9.16 (2H, s, Ph-H), $5.36(1 \mathrm{H}, \mathrm{d}, J=3.6 \mathrm{~Hz}, \mathrm{SiOCH}), 2.56-2.61(1 \mathrm{H}, \mathrm{m}, \mathrm{CHCO}), 2.04-$ 2.12 (2H, m, - $\mathrm{CH}-), 1.49-1.86$ (9H, m, - $\mathrm{CH}-), 1.16-1.43$ (10H, m, -

$\mathrm{CH}-) ;{ }^{13} \mathrm{C}$ NMR $\left(100 \mathrm{MHz}, \mathrm{CDCl}_{3}\right) \delta 207.8,162.0,148.6,133.5$, $129.4,122.5,83.3,47.5,38.7,30.2,29.6,27.3,27.2,26.2,25.9,25.8,25.7,25.5,25.1$; HPLC condition: DAICEL Chiralpak AD-H, hexane $/ i-\mathrm{PrOH}=95: 5$, flow rate $=0.5$ $\mathrm{mL} / \mathrm{min}, \lambda=254 \mathrm{~nm}$, retention time: $22.8 \mathrm{~min}$ (minor), $35.0 \mathrm{~min}$ (major). 


\section{Preparation of Differently $\alpha, \alpha$-Disubstituted $\alpha$-Siloxy Aldehydes 5}

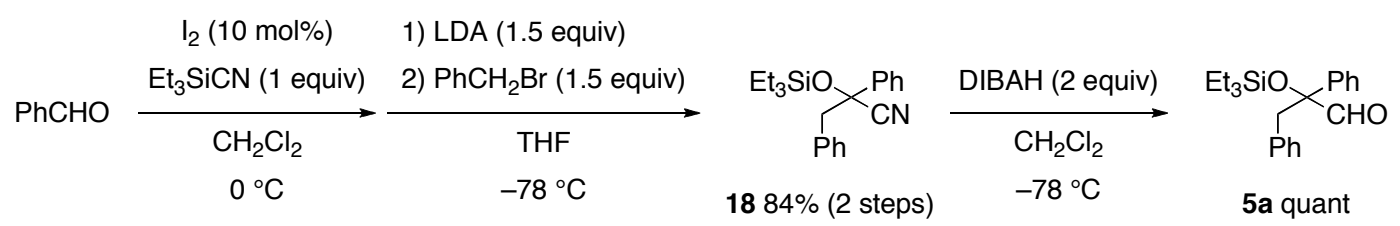

To a solution of benzaldehyde $(1.0 \mathrm{~mL}, 10 \mathrm{mmol})$ and triethylsilyl cyanide $(1.41 \mathrm{~g}, 10$ mmol) in $\mathrm{CH}_{2} \mathrm{Cl}_{2}(30 \mathrm{~mL})$ was added $\mathrm{I}_{2}(253 \mathrm{mg}, 1 \mathrm{mmol})$ at $0{ }^{\circ} \mathrm{C}$ under argon. After stirring for $30 \mathrm{~min}$ at $0{ }^{\circ} \mathrm{C}$, the reaction was quenched with saturated $\mathrm{Na}_{2} \mathrm{SO}_{3}$ and extractive workup was conducted with ether. The organic extracts were then washed with brine and dried over anhydrous $\mathrm{Na}_{2} \mathrm{SO}_{4}$. After evaporation of solvents, the crude products were dried under vacuum. To a solution of diisopropylamine $(2.1 \mathrm{~mL}, 15$ $\mathrm{mmol})$ in THF ( $15 \mathrm{~mL})$ was added $n$-BuLi $(10 \mathrm{~mL} ; 1.5 \mathrm{M}$ hexane solution, $15 \mathrm{mmol})$ at $-78{ }^{\circ} \mathrm{C}$ under argon. This solution was kept for $15 \mathrm{~min}$ with stirring, then slowly transferred into a THF $(15 \mathrm{~mL})$ solution of crude products at $-78{ }^{\circ} \mathrm{C}$. After stirring for $30 \mathrm{~min}$, benzyl bromide $(1.8 \mathrm{~mL}, 15 \mathrm{mmol})$ was added. The whole reaction mixture was stirred for $3 \mathrm{~h}$ at the same temperature and quenched with water. Extractive workup was performed with EtOAc and the combined extracts were washed with brine and dried over anhydrous $\mathrm{Na}_{2} \mathrm{SO}_{4}$. After concentration, the residual oil was purified by column chromatography on silica gel (EtOAc/hexane $=1 / 10$ as eluant) to afford $\mathbf{1 8}(2.83 \mathrm{~g}, 8.4$ mmol, $84 \%$ yield) as colorless oil. ${ }^{1} \mathrm{H}$ NMR $\left(400 \mathrm{MHz}, \mathrm{CDCl}_{3}\right) \delta 7.21-7.39(8 \mathrm{H}, \mathrm{m}, \mathrm{Ph}-$ H), 7.09-7.11 (2H, m, Ph-H), $3.24\left(1 \mathrm{H}, \mathrm{d}, J=13.6 \mathrm{~Hz}, \mathrm{PhCH}_{2}\right), 3.12(1 \mathrm{H}, \mathrm{d}, J=13.6$ $\left.\mathrm{Hz}, \mathrm{PhCH}_{2}\right), 0.81\left(9 \mathrm{H}, \mathrm{t}, J=8.0 \mathrm{~Hz}, \mathrm{CH}_{3} \mathrm{CH}_{2} \mathrm{Si}\right), 0.48-0.63\left(6 \mathrm{H}, \mathrm{m}, \mathrm{CH}_{3} \mathrm{CH}_{2} \mathrm{Si}\right)$.

To a solution of 18 in $\mathrm{CH}_{2} \mathrm{Cl}_{2}(16.8 \mathrm{~mL})$ was added DIBAH $(16.8 \mathrm{~mL} ; 1 \mathrm{M}$ toluene solution, $16.8 \mathrm{mmol}$ ) at $-78{ }^{\circ} \mathrm{C}$, and the reaction mixture was stirred for $1 \mathrm{~h}$ at the same temperature. Then, the mixture was quenched with $1 \mathrm{~N} \mathrm{HCl}$ and extracted with ether. The organic extracts were washed with brine and dried over anhydrous $\mathrm{Na}_{2} \mathrm{SO}_{4}$, and concentrated. The residual oil was purified by column chromatography on silica gel $\left(\mathrm{CH}_{2} \mathrm{Cl}_{2} /\right.$ hexane $=1 / 4$ as eluant $)$ to give $5 \mathbf{a}(2.86 \mathrm{~g}, 8.4 \mathrm{mmol}$, quant $)$ as colorless oil.

Other substrates $(\mathbf{5 b}, \mathbf{5} \mathbf{c}$ and $\mathbf{5 d})$ were synthesized by the same procedure.

5e was synthesized from a known ketone by the procedure described in S2. 
X. General Procedure for Kinetic Resolution of $\alpha, \alpha$-Disubstituted $\alpha$-Siloxy Aldehydes via Chiral Organoaluminum Catalyzed 1,2-Rearrangement

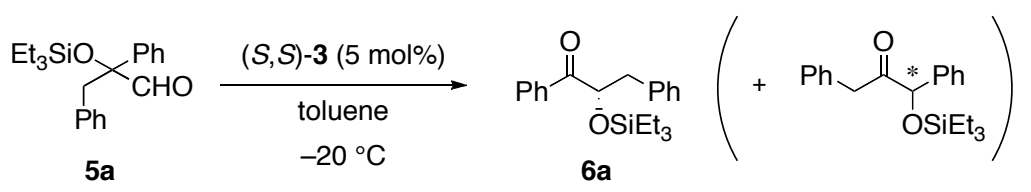

To a solution of 2-hydroxy-2'-[3,5-bis(trifluoromethyl)]phenyl-3-\{2-[3,5bis(trifluoromethyl)]benzenesulfonylamino\}phenyl-1,1'-binaphthyl (4, $46.7 \mathrm{mg}, 0.055$ $\mathrm{mmol})$ in toluene $(10 \mathrm{~mL})$ was added a $1 \mathrm{M}$ toluene solution of trimethylaluminum (50 $\mu \mathrm{L}, 0.050 \mathrm{mmol}$ ) at room temperature under nitrogen and stirred for $30 \mathrm{~min}$. After being cooled to $-20^{\circ} \mathrm{C}, \mathbf{5 a}(354.5 \mathrm{mg}, 1 \mathrm{mmol})$ was added to this solution and the stirring was maintained at $-20{ }^{\circ} \mathrm{C}$ for $12 \mathrm{~h}$. Then, sodium fluoride $(8.4 \mathrm{mg}, 0.20 \mathrm{mmol})$ and water $(2.7 \mu \mathrm{L}, 0.15 \mathrm{mmol})$ were added and the whole mixture was stirred for $30 \mathrm{~min}$ at room temperature. To remove precipitates, filtration through celite with EtOAc was carried out. Concentration of the filtrate and purification by column chromatography on silica gel $\left(\mathrm{CH}_{2} \mathrm{Cl} /\right.$ hexane $=1 / 4$ as eluant $)$ afforded $6 \mathbf{a}(166.9 \mathrm{mg}, 0.49 \mathrm{mmol}, 49 \%$ yield, The ratio of $\mathbf{6 a} /$ the product through the migration of phenyl group was $=>20: 1$ ) and $\mathbf{5 a}$ (173.7 $\mathrm{mg}, 0.51 \mathrm{mmol}, 51 \%$ recovery).

\section{Charactarization of 5 and 6}

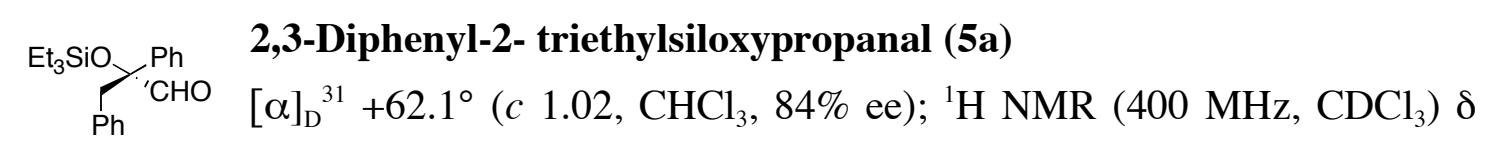
9.77 (1H, s, CHO), 7.26-7.33 (5H, m, Ph-H), 7.12-7.14 (3H, m, Ph-H), 6.95-6.98 (2H, m, Ph-H), $3.32\left(1 \mathrm{H}, \mathrm{d}, J=14.2 \mathrm{~Hz}, \mathrm{PhCH}_{2}\right), 3.25\left(1 \mathrm{H}, \mathrm{d}, J=14.2 \mathrm{~Hz}, \mathrm{PhCH}_{2}\right), 0.89(9 \mathrm{H}$, $\left.\mathrm{t}, J=8.0 \mathrm{~Hz}, \mathrm{C}_{3} \mathrm{CH}_{2} \mathrm{Si}\right), 0.58\left(6 \mathrm{H}, \mathrm{q}, J=8.0 \mathrm{~Hz}, \mathrm{CH}_{3} \mathrm{C}_{2} \mathrm{Si}\right) ;{ }^{13} \mathrm{C} \mathrm{NMR}(100 \mathrm{MHz}$, $\left.\mathrm{CDCl}_{3}\right) \delta 199.8,139.4,135.4,130.7,128.2$, 127.6, 127.5, 126.3, 126.0, 85.1, 44.9, 7.0, 6.8; IR (neat) 2954, 2937, 2910, 2875, 2808, 1734, 1496, 1454, 1242, 1143, 1076, 1008, 956, 761, 729, $698 \mathrm{~cm}^{-1}$; HRMS (ESI-TOF) Calcd. for $\mathrm{C}_{21} \mathrm{H}_{28} \mathrm{NaO}_{2} \mathrm{Si}\left([\mathrm{M}+\mathrm{Na}]^{+}\right)$: 363.1751, Found: 363.1762. Enantiomeric excess was determined by HPLC analysis after desilylation as described below.

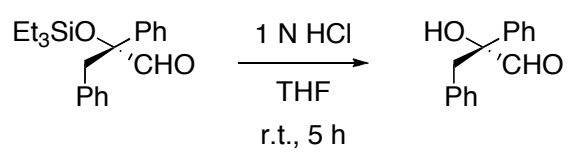

To a solution of $5 \mathbf{a}(68.1 \mathrm{mg}, 0.2 \mathrm{mmol})$ in THF $(2 \mathrm{~mL})$ was added $1 \mathrm{~N} \mathrm{HCl}(1 \mathrm{~mL})$ at room temperature. After vigorous stirring for $5 \mathrm{~h}$, this mixture was treated with saturated $\mathrm{NaHCO}_{3}$ and extractive workup was conducted with EtOAc. The combined 
organics were washed with brine and dried over anhydrous $\mathrm{Na}_{2} \mathrm{SO}_{4}$. Removal of solvents and purification of the residual oil by short column chromatography gave 2,3diphenyl-2-hydroxypropanal (45.3 $\mathrm{mg}, 0.2 \mathrm{mmol}$, quant).

$$
\begin{array}{ll}
{ }^{1} \mathrm{HONMR}\left(400 \mathrm{MHz}, \mathrm{CDCl}_{3}\right) \delta 9.72(1 \mathrm{H}, \mathrm{s}, \mathrm{CHO}), 7.55(2 \mathrm{H}, \mathrm{d}, J=8.0 \mathrm{~Hz}, \\
{ }_{\mathrm{Ph}}{ }^{\mathrm{C}} \mathrm{CHO} & \mathrm{Ph}-\mathrm{H}), 7.42(2 \mathrm{H}, \mathrm{t}, J=8.0 \mathrm{~Hz}, \mathrm{Ph}-\mathrm{H}), 7.34(1 \mathrm{H}, \mathrm{t}, J=7.6 \mathrm{~Hz}, \mathrm{Ph}-\mathrm{H}), 7.22-
\end{array}
$$
7.27 (3H, m, Ph-H), $7.15(2 \mathrm{H}, \mathrm{d}, J=7.6 \mathrm{~Hz}, \mathrm{Ph}-\mathrm{H}), 3.57(1 \mathrm{H}, \mathrm{s}, \mathrm{OH}), 3.53(2 \mathrm{H}, \mathrm{d}, J=$ $\left.14.0 \mathrm{~Hz}, \mathrm{PhCH}_{2}\right), 3.26\left(2 \mathrm{H}, \mathrm{d}, J=14.0 \mathrm{~Hz}, \mathrm{PhCH}_{2}\right) ;{ }^{13} \mathrm{C} \mathrm{NMR}(100 \mathrm{MHz}$, $\left.\mathrm{CDCl}_{3}\right) \delta 199.7,138.2,134.4,130.4,128.8,128.3,128.1,127.1,125.8,81.8,43.7$; HPLC condition: DAICEL Chiralpak AD-H, hexane $/ i$-PrOH $=95: 5$, flow rate $=0.5$ $\mathrm{mL} / \mathrm{min}, \lambda=254 \mathrm{~nm}$, retention time: $26.7 \min (S), 28.9 \min (R)$.

Absolute configuration was established by comparison of optical rotation with known literature value ${ }^{4}$ after transformation as described below.

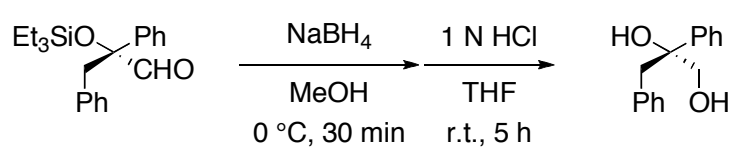

To a solution of $5 \mathbf{a}(68.1 \mathrm{mg}, 0.2 \mathrm{mmol}, 84 \%$ ee $)$ in $\mathrm{MeOH}(2 \mathrm{~mL})$ was added $\mathrm{NaBH}_{4}$ (15.1 mg, $0.4 \mathrm{mmol}$ ) at $0{ }^{\circ} \mathrm{C}$, and the reaction mixture was kept for $30 \mathrm{~min}$ with stirring. Then this mixture was quenched with water and extracted with EtOAc. The organic extracts were washed with brine and dried over anhydrous $\mathrm{Na}_{2} \mathrm{SO}_{4}$. After evaporation, the crude mixture was dissolved into THF $(2 \mathrm{~mL})$, and $1 \mathrm{~N} \mathrm{HCl}(1 \mathrm{~mL})$ was added at room temperature. The whole mixture was stirred vigorously for $5 \mathrm{~h}$ and quenched with saturated $\mathrm{NaHCO}_{3}$. Extractive workup was performed with EtOAc, and the combined extracts were washed with brine and dried over anhydrous $\mathrm{Na}_{2} \mathrm{SO}_{4}$. Removal of solvents and purification of the residual oil afforded 2,3-diphenylpropan-1,2-diol ${ }^{4}$ (43.3 mg, 0.19 mmol, $95 \%)$.

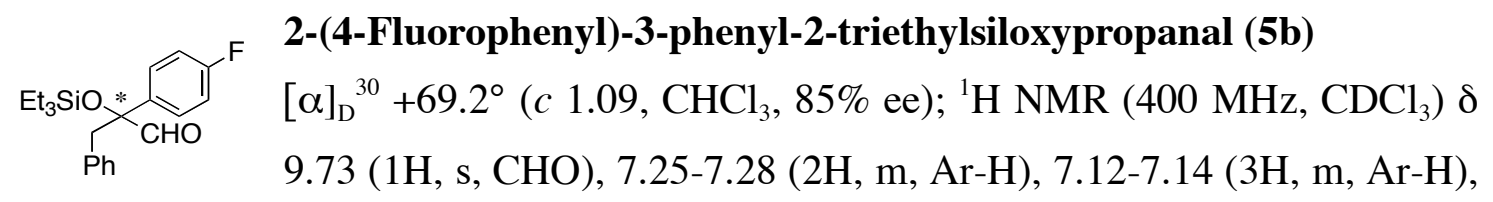
6.92-7.02 (4H, m, Ar-H), $3.28\left(1 \mathrm{H}, \mathrm{d}, J=14.2 \mathrm{~Hz}, \mathrm{PhCH}_{2}\right), 3.20(1 \mathrm{H}, \mathrm{d}, J=14.2 \mathrm{~Hz}$, $\left.\mathrm{PhCH}_{2}\right), 0.91\left(9 \mathrm{H}, \mathrm{t}, J=8.0 \mathrm{~Hz}, \mathrm{CH}_{3} \mathrm{CH}_{2} \mathrm{Si}\right), 0.59\left(6 \mathrm{H}, \mathrm{q}, J=8.0 \mathrm{~Hz}, \mathrm{CH}_{3} \mathrm{CH}_{2} \mathrm{Si}\right) ;{ }^{13} \mathrm{C}$ NMR $\left(100 \mathrm{MHz} \mathrm{CDCl}_{3}\right) \delta 199.5,162.2\left(\mathrm{~d}, J_{\mathrm{C}-\mathrm{F}}=247 \mathrm{~Hz}\right), 135.1,135.0,130.6,127.8$ $\left(\mathrm{d}, J_{\mathrm{C}-\mathrm{F}}=8.3 \mathrm{~Hz}\right), 127.6,126.4,115.1\left(\mathrm{~d}, J_{\mathrm{C}-\mathrm{F}}=21.4 \mathrm{~Hz}\right), 84.7,44.9,6.9,6.8$; IR (neat) 2954, 2912, 2875, 2808, 1734, 1602, 1506, 1456, 1236, 1159, 1095, 1012, 956, 833, 740, 729, 698, $669 \mathrm{~cm}^{-1}$; HRMS (ESI-TOF) Calcd. for $\mathrm{C}_{21} \mathrm{H}_{27} \mathrm{FNaO}_{2} \mathrm{Si}\left([\mathrm{M}+\mathrm{Na}]^{+}\right)$: 381.1656, Found: 381.1653. Enantiomeric excess was determined by HPLC analysis after desilylation. 


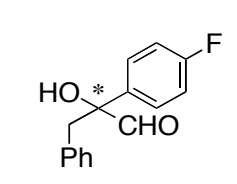

${ }^{1} \mathrm{H}$ NMR (400 MHz, $\mathrm{CDCl}_{3}$ ) $\delta 9.64$ (1H, s, CHO), 7.50-7.54 (2H, m, ArH), 7.22-7.26 (3H, m, Ar-H), 7.08-7.14 (4H, m, Ar-H), 3.56 (1H, s, OH), $3.49\left(2 \mathrm{H}, \mathrm{d}, J=14.0 \mathrm{~Hz}, \mathrm{PhCH}_{2}\right), 3.23\left(2 \mathrm{H}, \mathrm{d}, J=14.0 \mathrm{~Hz}, \mathrm{PhCH}_{2}\right) ;{ }^{13} \mathrm{C}$ NMR (100 MHz, $\left.\mathrm{CDCl}_{3}\right) \delta 199.4,162.5\left(\mathrm{~d}, J_{\mathrm{C}-\mathrm{F}}=248 \mathrm{~Hz}\right), 134.1,133.9\left(\mathrm{~d}, J_{\mathrm{C}-\mathrm{F}}=3.3\right.$ $\mathrm{Hz}), 130.4,128.3,127.7\left(\mathrm{~d}, J_{\mathrm{C}-\mathrm{F}}=8.2 \mathrm{~Hz}\right), 127.2,115.7\left(\mathrm{~d}, J_{\mathrm{C}-\mathrm{F}}=21.4 \mathrm{~Hz}\right), 81.4,43.7$; HPLC condition: DAICEL Chiralpak AD-H, hexane $/ i$-PrOH $=97: 3$, flow rate $=0.5$ $\mathrm{mL} / \mathrm{min}, \lambda=233 \mathrm{~nm}$, retention time: $36.3 \mathrm{~min}$ (major), $54.0 \mathrm{~min}$ (minor).

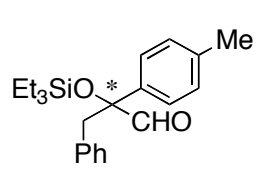

\section{2-(4-Methylphenyl)-3-phenyl-2-triethylsiloxypropanal (5c)}

$[\alpha]_{\mathrm{D}}{ }^{28}+52.9^{\circ}\left(c 0.89, \mathrm{CHCl}_{3}, 83 \%\right.$ ee $) ;{ }^{1} \mathrm{H} \mathrm{NMR}\left(400 \mathrm{MHz}, \mathrm{CDCl}_{3}\right) \delta$ 9.73 (1H, s, CHO), 7.11-7.26 (7H, m, Ar-H), 6.97-6.99 (2H, m, ArH), $3.29\left(1 \mathrm{H}, \mathrm{d}, J=14.4 \mathrm{~Hz}, \mathrm{PhCH}_{2}\right), 3.24\left(1 \mathrm{H}, \mathrm{d}, J=14.4 \mathrm{~Hz}, \mathrm{PhCH}_{2}\right), 2.32(3 \mathrm{H}, \mathrm{s}$, $\mathrm{Me}-\mathrm{Ph}), 0.88\left(9 \mathrm{H}, \mathrm{t}, J=8.0 \mathrm{~Hz}, \mathrm{CH}_{3} \mathrm{CH}_{2} \mathrm{Si}\right), 0.56\left(6 \mathrm{H}, \mathrm{q}, J=8.0 \mathrm{~Hz}, \mathrm{CH}_{3} \mathrm{CH}_{2} \mathrm{Si}\right) ;{ }^{13} \mathrm{C}$ NMR $\left(100 \mathrm{MHz}, \mathrm{CDCl}_{3}\right) \delta 199.9,137.3,136.4,135.6,130.7,129.0,127.5,126.2,126.0$, 85.0, 44.8, 20.9, 7.0, 6.8; IR (neat) 2954, 2916, 2875, 1732, 1454, 1242, 1151, 1112, 1010, 958, 812, 736, 729, $698 \mathrm{~cm}^{-1}$; HRMS (ESI-TOF) Calcd. for $\mathrm{C}_{22} \mathrm{H}_{30} \mathrm{NaO}_{2} \mathrm{Si}$ $\left([\mathrm{M}+\mathrm{Na}]^{+}\right):$377.1907, Found: 377.1908. Enantiomeric excess was determined by HPLC analysis after desilylation.

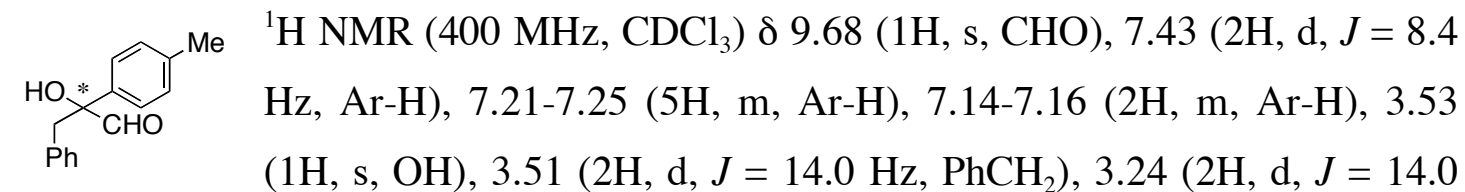

$\left.\mathrm{Hz}, \mathrm{PhCH}_{2}\right) ;{ }^{13} \mathrm{C} \mathrm{NMR}\left(100 \mathrm{MHz}, \mathrm{CDCl}_{3}\right) \delta 199.6,137.7,135.1,134.5,130.3,129.4$, 128.1, 126.9, 125.7, 81.6, 43.4, 20.9; HPLC condition: DAICEL Chiralcel OJ-H, hexane $/ i-\mathrm{PrOH}=95: 5$, flow rate $=1.0 \mathrm{~mL} / \mathrm{min}, \lambda=223 \mathrm{~nm}$, retention time: $18.7 \mathrm{~min}$ (minor), $25.3 \mathrm{~min}$ (major).

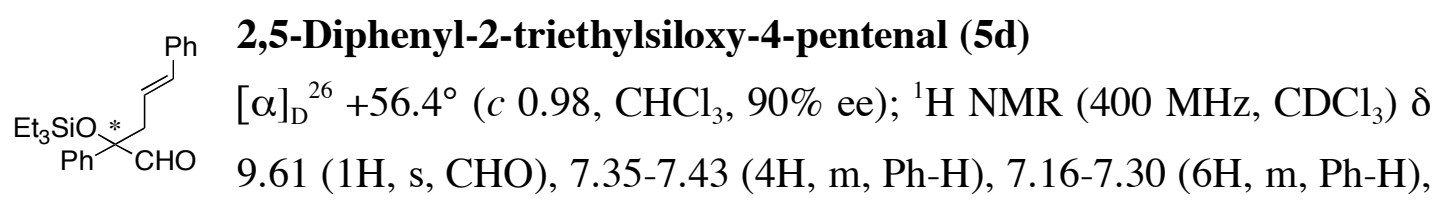
$6.35(1 \mathrm{H}, \mathrm{d}, J=16.0 \mathrm{~Hz}, \mathrm{CH}=\mathrm{C} \underline{\mathrm{HPh}}), 5.96\left(1 \mathrm{H}, \mathrm{dt}, J=16.0,7.2 \mathrm{~Hz}, \mathrm{CH}_{2} \mathrm{C} \underline{\mathrm{H}}=\mathrm{CH}\right), 3.08$ $\left(1 \mathrm{H}, \mathrm{dd}, J=15.2,7.2 \mathrm{~Hz}, \mathrm{CH}=\mathrm{CHCH}_{2}\right), 2.84\left(1 \mathrm{H}, \mathrm{dd}, J=15.2,7.2 \mathrm{~Hz}, \mathrm{CH}=\mathrm{CHCH}_{2}\right)$, $0.98\left(9 \mathrm{H}, \mathrm{t}, J=7.6 \mathrm{~Hz}, \mathrm{CH}_{3} \mathrm{CH}_{2} \mathrm{Si}\right), 0.68\left(6 \mathrm{H}, \mathrm{q}, J=7.6 \mathrm{~Hz}, \mathrm{CH}_{3} \mathrm{CH}_{2} \mathrm{Si}\right) ;{ }^{13} \mathrm{C} \mathrm{NMR}(100$ $\left.\mathrm{MHz} \mathrm{CDCl}_{3}\right) \delta 199.7,138.7,137.3,133.6,128.5,128.3,127.8,127.0,126.1,125.9$, 123.8, 84.6, 41.1, 7.0, 6.7; IR (neat) 3026, 2954, 2910, 2875, 2802, 1734, 1494, 1458, 1240, 1139, 1074, 1014, 964, 840, 740, 729, $698 \mathrm{~cm}^{-1}$; HRMS (ESI-TOF) Calcd. for $\mathrm{C}_{23} \mathrm{H}_{30} \mathrm{NaO}_{2} \mathrm{Si}\left([\mathrm{M}+\mathrm{Na}]^{+}\right)$: 389.1907, Found: 389.1922. Enantiomeric excess was determined by HPLC analysis after desilylation. 


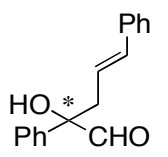

${ }^{1} \mathrm{H}$ NMR (400 MHz, $\left.\mathrm{CDCl}_{3}\right) \delta 9.65(1 \mathrm{H}, \mathrm{s}, \mathrm{CHO}), 7.53(2 \mathrm{H}, \mathrm{d}, J=8.0$ $\mathrm{Hz}, \mathrm{Ph}-\mathrm{H}), 7.42$ (2H, t $J=8.0 \mathrm{~Hz}, \mathrm{Ph}-\mathrm{H}), 7.19-7.35$ (6H, m, Ph-H), 6.53 $(1 \mathrm{H}, \mathrm{d}, J=16.0 \mathrm{~Hz}, \mathrm{CH}=\mathrm{CHPh}), 6.08(1 \mathrm{H}, \mathrm{dt}, J=16.0,7.6 \mathrm{~Hz}$, $\left.\mathrm{CH}_{2} \mathrm{C} \underline{\mathrm{H}}=\mathrm{CH}\right), 3.75(1 \mathrm{H}, \mathrm{s}, \mathrm{OH}), 3.04\left(1 \mathrm{H}, \mathrm{dd}, J=14.4,7.6 \mathrm{~Hz}, \mathrm{CH}=\mathrm{CHC}_{2}\right), 2.96(1 \mathrm{H}$, $\left.\mathrm{dd}, J=14.4,7.6 \mathrm{~Hz}, \mathrm{CH}=\mathrm{CHCH}_{2}\right) ;{ }^{13} \mathrm{C} \mathrm{NMR}\left(100 \mathrm{MHz}, \mathrm{CDCl}_{3}\right) \delta 199.8,138.0,136.6$, 135.0, 128.8, 128.4, 128.1, 127.5, 126.2, 125.7, 122.2, 81.3, 40.9; HPLC condition: DAICEL Chiralcel OJ-H, hexane $/ i-\mathrm{PrOH}=95: 5$, flow rate $=1.0 \mathrm{~mL} / \mathrm{min}, \lambda=254 \mathrm{~nm}$, retention time: 33.9 min (major), $40.2 \mathrm{~min}$ (minor).

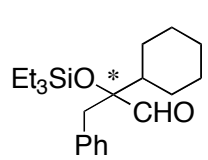

2-Cyclohexyl-3-phenyl-2-triethylsiloxypropanal (5e)

$[\alpha]_{\mathrm{D}}{ }^{21}+7.6^{\circ}\left(c 0.96, \mathrm{CHCl}_{3}, 88 \%\right.$ ee $) ;{ }^{1} \mathrm{H} \mathrm{NMR}\left(400 \mathrm{MHz}, \mathrm{CDCl}_{3}\right) \delta$ $9.58(1 \mathrm{H}, \mathrm{s}, \mathrm{CHO}), 7.17-7.26(3 \mathrm{H}, \mathrm{m}, \mathrm{Ph}-\mathrm{H}), 7.13(2 \mathrm{H}, \mathrm{d}, J=6.8 \mathrm{~Hz}$, $\mathrm{Ph}-\mathrm{H}), 3.09\left(1 \mathrm{H}, \mathrm{d}, J=14.0 \mathrm{~Hz}, \mathrm{PhCH}_{2}\right), 2.86\left(1 \mathrm{H}, \mathrm{d}, J=14.0 \mathrm{~Hz}, \mathrm{PhCH}_{2}\right), 1.52-1.95$ $(5 \mathrm{H}, \mathrm{m},-\mathrm{CH}-), 1.14-1.26(6 \mathrm{H}, \mathrm{m},-\mathrm{CH}-), 0.88$ (9H, t $\left., J=8.0 \mathrm{~Hz}, \mathrm{C}_{3} \mathrm{CH}_{2} \mathrm{Si}\right), 0.43-0.60$ $\left(6 \mathrm{H}, \mathrm{m}, \mathrm{CH}_{3} \mathrm{C}_{2} \mathrm{Si}\right) ;{ }^{13} \mathrm{C} \mathrm{NMR}\left(100 \mathrm{MHz}, \mathrm{CDCl}_{3}\right) \delta 204.6,136.0,130.5,127.9,126.4$, 85.6, 43.1, 40.4, 27.1, 26.5, 26.4, 26.3, 7.2, 6.9 (one signal for cyclohexyl carbon was not identified due to the overlap of the peaks); IR (neat) 2931, 2873, 2854, 2816, 1737 , 1454, 1240, 1170, 1139, 1010, 964, 740, $700 \mathrm{~cm}^{-1}$; HRMS (ESI-TOF) Calcd. for $\mathrm{C}_{21} \mathrm{H}_{34} \mathrm{NaO}_{2} \mathrm{Si}\left([\mathrm{M}+\mathrm{Na}]^{+}\right)$: 369.2220, Found: 369.2218; HPLC condition: DAICEL Chiralpak AD-H, hexane $/ i-\mathrm{PrOH}=99: 1$, flow rate $=0.3 \mathrm{~mL} / \mathrm{min}, \lambda=220 \mathrm{~nm}$, retention time: $14.0 \mathrm{~min}$ (minor), $17.0 \mathrm{~min}$ (major).

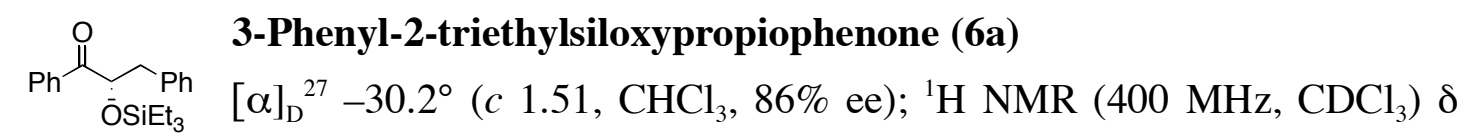
8.05-8.07 (2H, m, Ph-H), 7.56 (1H, t, $J=7.6 \mathrm{~Hz}, \mathrm{Ph}-\mathrm{H}), 7.45$ (2H, t, $J=7.6 \mathrm{~Hz}, \mathrm{Ph}-\mathrm{H})$, 7.21-7.29 (5H, m, Ph-H), 4.95 (1H, dd, $J=9.0,4.0 \mathrm{~Hz}, \mathrm{SiOCH}), 3.10$ (1H, dd, $J=13.6$, $\left.4.0 \mathrm{~Hz}, \mathrm{PhCH}_{2}\right), 3.00\left(1 \mathrm{H}, \mathrm{dd}, J=13.6,9.0 \mathrm{~Hz}, \mathrm{PhCH}_{2}\right), 0.75(9 \mathrm{H}, \mathrm{t}, J=8.0 \mathrm{~Hz}$, $\left.\mathrm{C}_{3} \mathrm{CH}_{2} \mathrm{Si}\right), 0.39\left(6 \mathrm{H}, \mathrm{q}, J=8.0 \mathrm{~Hz}, \mathrm{CH}_{3} \mathrm{CH}_{2} \mathrm{Si}\right) ;{ }^{13} \mathrm{C} \mathrm{NMR}\left(100 \mathrm{MHz}, \mathrm{CDCl}_{3}\right) \delta 200.7$, $137.5,134.9,133.0,129.6,129.1,128.3,128.1,126.5,78.6,42.2,6.4,4.4$; IR (neat) 2954, 2910, 2875, 1697, 1680, 1454, 1278, 1240, 1109, 1004, 771, 744, 731, $698 \mathrm{~cm}^{-1}$; HRMS (ESI-TOF) Calcd. for $\mathrm{C}_{21} \mathrm{H}_{28} \mathrm{NaO}_{2} \mathrm{Si}\left([\mathrm{M}+\mathrm{Na}]^{+}\right)$: 363.1751, Found: 363.1749; HPLC condition: DAICEL Chiralcel OD-H, hexane $/ i-\mathrm{PrOH}=99: 1$, flow rate $=0.3$ $\mathrm{mL} / \mathrm{min}, \lambda=210 \mathrm{~nm}$, retention time: $15.4 \min (S), 16.9 \min (R)$.

Absolute configuration was established by comparison of optical rotation with known literature value ${ }^{5}$ after desilylation. 


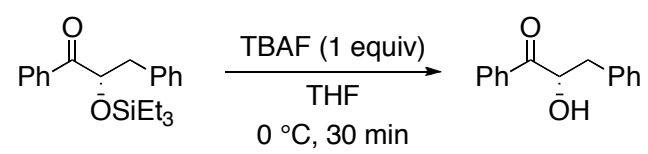

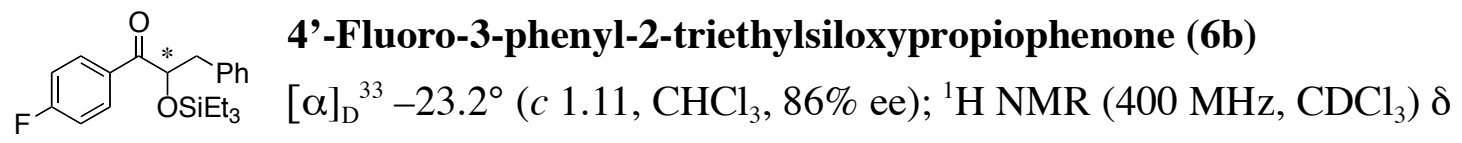
8.10-8.14 (2H, m, Ar-H), 7.20-7.29 (5H, m, Ar-H), 7.11 (2H, t, $J=8.4$ Hz, Ar-H), 4.84 $(1 \mathrm{H}, \mathrm{dd}, J=8.4,4.8 \mathrm{~Hz}, \mathrm{SiOCH}), 3.09\left(1 \mathrm{H}, \mathrm{dd}, J=13.6,4.8 \mathrm{~Hz}, \mathrm{PhCH}_{2}\right), 3.02(1 \mathrm{H}, \mathrm{dd}$, $\left.J=13.6,8.4 \mathrm{~Hz}, \mathrm{PhCH}_{2}\right), 0.75\left(9 \mathrm{H}, \mathrm{t}, J=8.0 \mathrm{~Hz}, \mathrm{C}_{3} \mathrm{CH}_{2} \mathrm{Si}\right), 0.39(6 \mathrm{H}, \mathrm{q}, J=8.0 \mathrm{~Hz}$, $\left.\mathrm{CH}_{3} \mathrm{CH}_{2} \mathrm{Si}\right) ;{ }^{13} \mathrm{C}$ NMR $\left(100 \mathrm{MHz}, \mathrm{CDCl}_{3}\right) \delta 199.4,165.5\left(\mathrm{~d}, J_{\mathrm{C}-\mathrm{F}}=256 \mathrm{~Hz}\right), 137.2,132.1$ $\left(\mathrm{d}, J_{\mathrm{C}-\mathrm{F}}=9.0 \mathrm{~Hz}\right), 131.2\left(\mathrm{~d}, J_{\mathrm{C}-\mathrm{F}}=2.5 \mathrm{~Hz}\right), 129.6,128.2,126.6,115.4\left(\mathrm{~d}, J_{\mathrm{C}-\mathrm{F}}=21.4 \mathrm{~Hz}\right)$, 79.4, 42.4, 6.4, 4.4; IR (neat) 2954, 2912, 2875, 1697, 1680, 1597, 1504, 1456, 1409, 1280, 1236, 1155, 1103, 1012, 981, 933, 840, 817, 777, 742, 729, $673 \mathrm{~cm}^{-1}$; HRMS (ESI-TOF) Calcd. for $\mathrm{C}_{21} \mathrm{H}_{27} \mathrm{FNaO}_{2} \mathrm{Si}\left([\mathrm{M}+\mathrm{Na}]^{+}\right)$: 381.1656, Found: 381.1667. Enantiomeric excess was determined by HPLC analysis after desilylation.

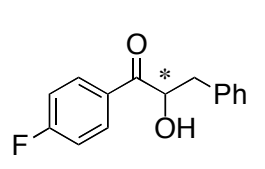

${ }^{1} \mathrm{H}$ NMR (400 MHz, $\mathrm{CDCl}_{3}$ ) $\delta$ 7.93-7.96 (2H, m, Ar-H), 7.16-7.27

$(5 \mathrm{H}, \mathrm{m}, \mathrm{Ar}-\mathrm{H}), 7.11(2 \mathrm{H}, \mathrm{d}, J=7.6 \mathrm{~Hz}, \mathrm{Ar}-\mathrm{H}), 5.29(1 \mathrm{H}, \mathrm{dt}, J=6.8$,

$4.4 \mathrm{~Hz}, \mathrm{HOC} \underline{\mathrm{H}}), 3.66(1 \mathrm{H}, \mathrm{d}, J=6.8 \mathrm{~Hz}, \mathrm{OH}), 3.17(1 \mathrm{H}, \mathrm{dd}, J=$ 14.2, 4.4 Hz, $\left.\mathrm{PhCH}_{2}\right), 2.91\left(1 \mathrm{H}, \mathrm{dd}, J=14.2,6.8 \mathrm{~Hz}, \mathrm{PhCH}_{2}\right) ;{ }^{13} \mathrm{C} \mathrm{NMR}(100 \mathrm{MHz}$, $\left.\mathrm{CDCl}_{3}\right) \delta 199.4,166.1\left(\mathrm{~d}, J_{\mathrm{C}-\mathrm{F}}=257 \mathrm{~Hz}\right), 136.1,131.3\left(\mathrm{~d}, J_{\mathrm{C}-\mathrm{F}}=9.1 \mathrm{~Hz}\right), 130.2\left(\mathrm{~d}, J_{\mathrm{C}-\mathrm{F}}=\right.$ $3.3 \mathrm{~Hz}), 129.3,128.3,126.8,116.1\left(\mathrm{~d}, J_{\mathrm{C}-\mathrm{F}}=22.3 \mathrm{~Hz}\right), 73.6,41.9$; HPLC condition: DAICEL Chiralpak AD-H, hexane $/ i-\mathrm{PrOH}=95: 5$, flow rate $=0.5 \mathrm{~mL} / \mathrm{min}, \lambda=254 \mathrm{~nm}$, retention time: $35.7 \mathrm{~min}$ (minor), $39.4 \mathrm{~min}$ (major).

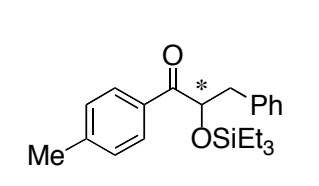

\section{4'-Methyl-3-phenyl-2-triethylsiloxypropiophenone (6c)}

$[\alpha]_{\mathrm{D}}^{30}-21.8^{\circ}$ (c 1.03, $\mathrm{CHCl}_{3}, 85 \%$ ee); ${ }^{1} \mathrm{H} \mathrm{NMR}\left(400 \mathrm{MHz}, \mathrm{CDCl}_{3}\right.$ )

$\delta 7.98(2 \mathrm{H}, \mathrm{d}, J=8.8 \mathrm{~Hz}, \mathrm{Ar}-\mathrm{H}), 7.16-7.29(7 \mathrm{H}, \mathrm{m}, \mathrm{Ar}-\mathrm{H}), 4.93$ $(1 \mathrm{H}, \mathrm{dd}, J=9.2,4.2 \mathrm{~Hz}, \mathrm{SiOCH}), 3.08\left(1 \mathrm{H}, \mathrm{dd}, J=13.6,4.2 \mathrm{~Hz}, \mathrm{PhCH}_{2}\right), 2.98(1 \mathrm{H}, \mathrm{dd}$, $\left.J=13.6,9.2 \mathrm{~Hz}, \mathrm{PhCH}_{2}\right), 2.42(3 \mathrm{H}, \mathrm{s}, \mathrm{Me}-\mathrm{Ph}), 0.75\left(9 \mathrm{H}, \mathrm{t}, J=8.0 \mathrm{~Hz}, \mathrm{C}_{3} \mathrm{CH}_{2} \mathrm{Si}\right), 0.39$ $\left(6 \mathrm{H}, \mathrm{q}, J=8.0 \mathrm{~Hz}, \mathrm{CH}_{3} \mathrm{CH}_{2} \mathrm{Si}\right) ;{ }^{13} \mathrm{C} \mathrm{NMR}\left(100 \mathrm{MHz}, \mathrm{CDCl}_{3}\right) \delta 200.3,143.9,137.8$, 132.4, 129.7, 129.3, 129.1, 128.2, 126.5, 78.6, 42.4, 21.7, 6.5, 4.5; IR (neat) 2954, 2916, 2875, 1697, 1678, 1606, 1456, 1280, 1238, 1180, 1111, 1016, 777, 744, $698 \mathrm{~cm}^{-1}$; HRMS (ESI-TOF) Calcd. for $\mathrm{C}_{22} \mathrm{H}_{30} \mathrm{NaO}_{2} \mathrm{Si}\left([\mathrm{M}+\mathrm{Na}]^{+}\right)$: 377.1907, Found: 377.1911; HPLC condition: DAICEL Chiralcel OJ-H, hexane $/ i-\mathrm{PrOH}=99: 1$, flow rate $=0.3$ $\mathrm{mL} / \mathrm{min}, \lambda=254 \mathrm{~nm}$, retention time: $14.7 \mathrm{~min}$ (minor), $17.1 \mathrm{~min}$ (major). 


\section{1,5-Diphenyl-2-triethylsiloxy-4-pentenone (6d)}

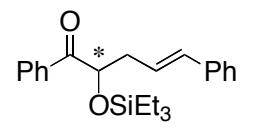

$[\alpha]_{\mathrm{D}}{ }^{27}-3.7^{\circ}\left(c 1.08, \mathrm{CHCl}_{3}, 63 \%\right.$ ee); ${ }^{1} \mathrm{H} \mathrm{NMR}\left(400 \mathrm{MHz}, \mathrm{CDCl}_{3}\right) \delta$

$8.07(2 \mathrm{H}, \mathrm{d}, J=7.2 \mathrm{~Hz}, \mathrm{Ph}-\mathrm{H}), 7.56(1 \mathrm{H}, \mathrm{t}, J=7.2 \mathrm{~Hz}, \mathrm{Ph}-\mathrm{H}), 7.46$

$(2 \mathrm{H}, \mathrm{t}, J=7.2 \mathrm{~Hz}, \mathrm{Ph}-\mathrm{H}), 7.18-7.32(5 \mathrm{H}, \mathrm{m}, \mathrm{Ph}-\mathrm{H}), 6.41(1 \mathrm{H}, \mathrm{d}, J=16.0 \mathrm{~Hz}$, $\mathrm{CH}=\mathrm{C} \underline{H P h}), 6.23\left(1 \mathrm{H}, \mathrm{dt}, J=16.0,7.2 \mathrm{~Hz}, \mathrm{CH}_{2} \mathrm{C} \underline{\mathrm{H}}=\mathrm{CH}\right), 4.91(1 \mathrm{H}, \mathrm{dd}, J=7.6,5.2 \mathrm{~Hz}$, $\mathrm{SiOCH}), 2.68-2.72\left(2 \mathrm{H}, \mathrm{m}, \mathrm{CH}=\mathrm{CHCH}_{2}\right), 0.90\left(9 \mathrm{H}, \mathrm{t}, J=8.0 \mathrm{~Hz}, \mathrm{C}_{3} \mathrm{CH}_{2} \mathrm{Si}\right), 0.58(6 \mathrm{H}$, q, $\left.J=8.0 \mathrm{~Hz}, \mathrm{CH}_{3} \underline{\mathrm{CH}}_{2} \mathrm{Si}\right) ;{ }^{13} \mathrm{C} \mathrm{NMR}\left(100 \mathrm{MHz}, \mathrm{CDCl}_{3}\right) \delta 200.5,137.2,135.0,133.0$, 132.9, 129.0, 128.4, 128.3, 127.1, 126.0, 125.2, 77.3, 39.6, 6.6, 4.7; IR (neat) 3026, 2954, 2937, 2910, 2875, 1697, 1678, 1597, 1448, 1415, 1274, 1238, 1118, 1002, 964 , 941, 923, 848, 829, 769, 742, $692 \mathrm{~cm}^{-1}$; HRMS (ESI-TOF) Calcd. for $\mathrm{C}_{23} \mathrm{H}_{30} \mathrm{NaO}_{2} \mathrm{Si}$ $\left([\mathrm{M}+\mathrm{Na}]^{+}\right)$: 389.1907, Found: 389.1902; HPLC condition: DAICEL Chiralcel OD-H, hexane $/ i-\mathrm{PrOH}=99: 1$, flow rate $=0.5 \mathrm{~mL} / \mathrm{min}, \lambda=254 \mathrm{~nm}$, retention time: $11.0 \mathrm{~min}$ (major), $13.1 \mathrm{~min}$ (minor).

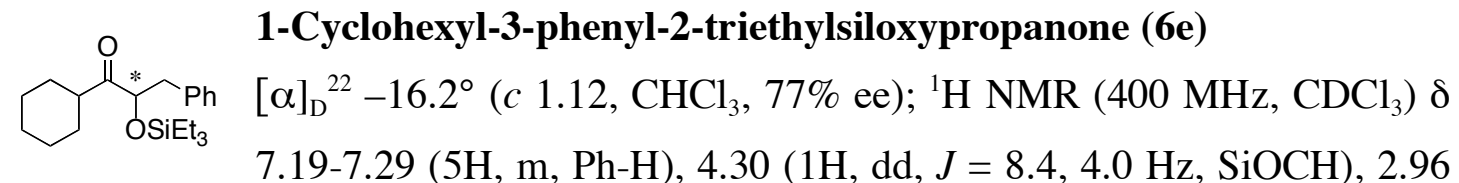
$\left(1 \mathrm{H}, \mathrm{dd}, J=13.6,4.0 \mathrm{~Hz}, \mathrm{PhCH}_{2}\right), 2.77\left(1 \mathrm{H}, \mathrm{dd}, J=13.6,8.4 \mathrm{~Hz}, \mathrm{PhCH}_{2}\right), 2.74-2.79$ $\left(1 \mathrm{H}, \mathrm{m},-\left(\mathrm{CH}_{2}\right)_{2} \mathrm{CHCO}\right), 1.65-1.75$ (5H, m, - $\left.\mathrm{CH}-\right), 1.16-1.37$ (5H, m, -CH-), 0.83 (9H, t, $\left.J=8.0 \mathrm{~Hz}, \mathrm{CH}_{3} \mathrm{CH}_{2} \mathrm{Si}\right), 0.36-0.47\left(6 \mathrm{H}, \mathrm{m}, \mathrm{CH}_{3} \mathrm{CH}_{2} \mathrm{Si}\right) ;{ }^{13} \mathrm{C} \mathrm{NMR}\left(100 \mathrm{MHz}, \mathrm{CDCl}_{3}\right)$ б 215.2, 137.5, 129.7, 128.1, 126.4, 79.4, 45.6, 41.3, 28.9, 28.0, 25.8, 25.7, 25.4, 6.6, 4.5; IR (neat) 2931, 2875, 2854, 1707, 1454, 1413, 1365, 1240, 1157, 1097, 1004, 937 , 773, 740, 729, $698 \mathrm{~cm}^{-1}$; HRMS (ESI-TOF) Calcd. for $\mathrm{C}_{21} \mathrm{H}_{34} \mathrm{NaO}_{2} \mathrm{Si}\left([\mathrm{M}+\mathrm{Na}]^{+}\right)$: 369.2220, Found: 369.2217; HPLC condition: DAICEL Chiralcel OD-H, hexane/iPrOH $=99: 1$, flow rate $=0.3 \mathrm{~mL} / \mathrm{min}, \lambda=220 \mathrm{~nm}$, retention time: $17.0 \mathrm{~min}$ (major), 36.6 min (minor).

\section{References}

(1) (a) Yang, H.; Hay, A. S. Synthesis 1992, 467. (b) Tanguy, G.; Weinberger, B.; Des Abbayes, H. Tetrahedron Lett. 1984, 25, 5529. (c) Lohmar, R.; Steglish, W. Chem. Ber. 1980, 113, 3706.

(2) Ooi, T.; Saito, A.; Maruoka, K. J. Am. Chem. Soc. 2003, 125, 3220.

(3) Ramachary, D. B.; Barbas III, C. F. Org. Lett. 2005, 7, 1577.

(4) Hof, R. P.; Kellogg, R. M. J. Chem. Soc., Perkin Trans. 1 1996, 2051.

(5) Giordani, A.; Carera, A.; Pinciroli, V.; Cozzi, P. Tetrahedron Asymmetry 1997, 8, 253. 

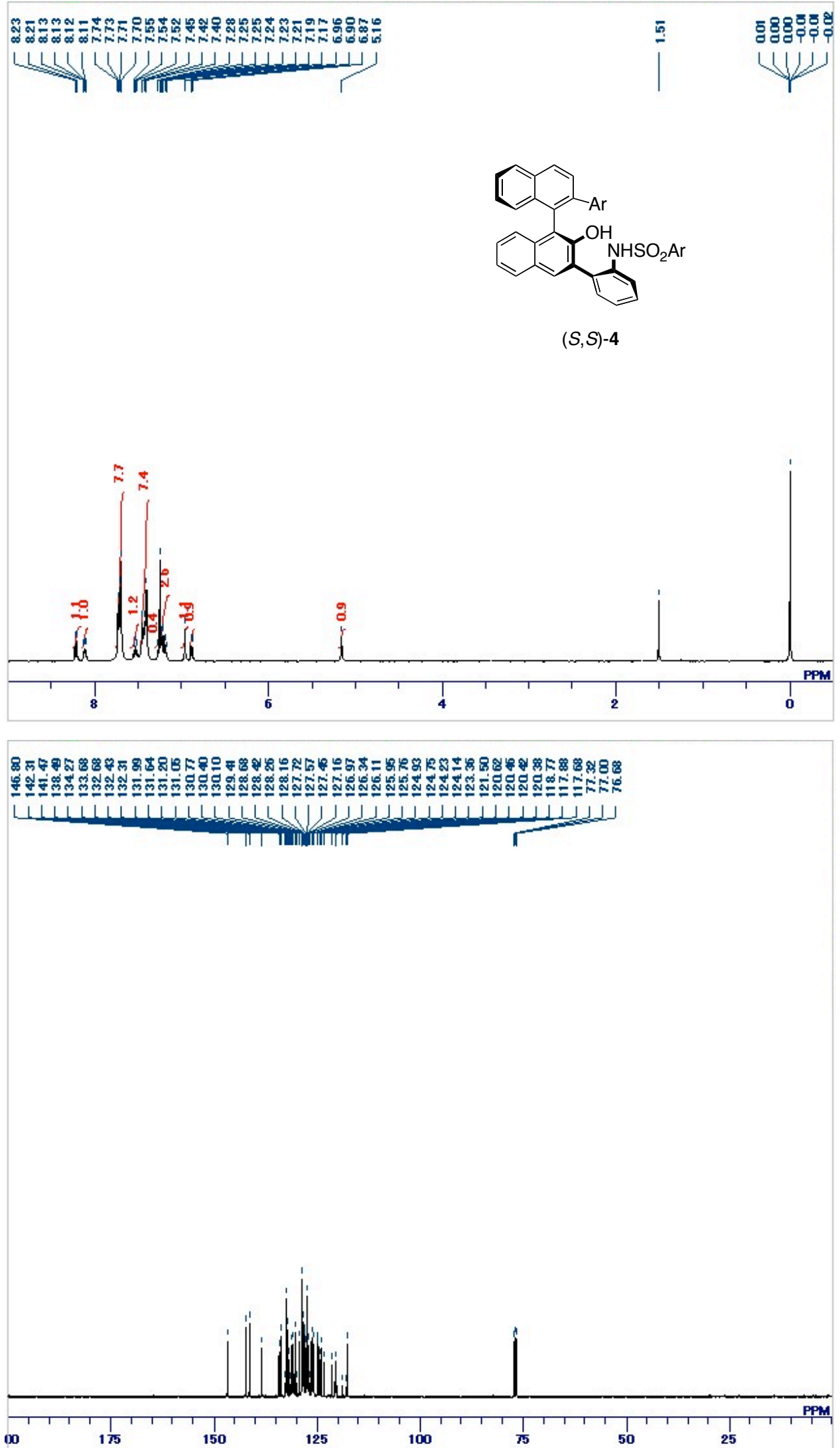


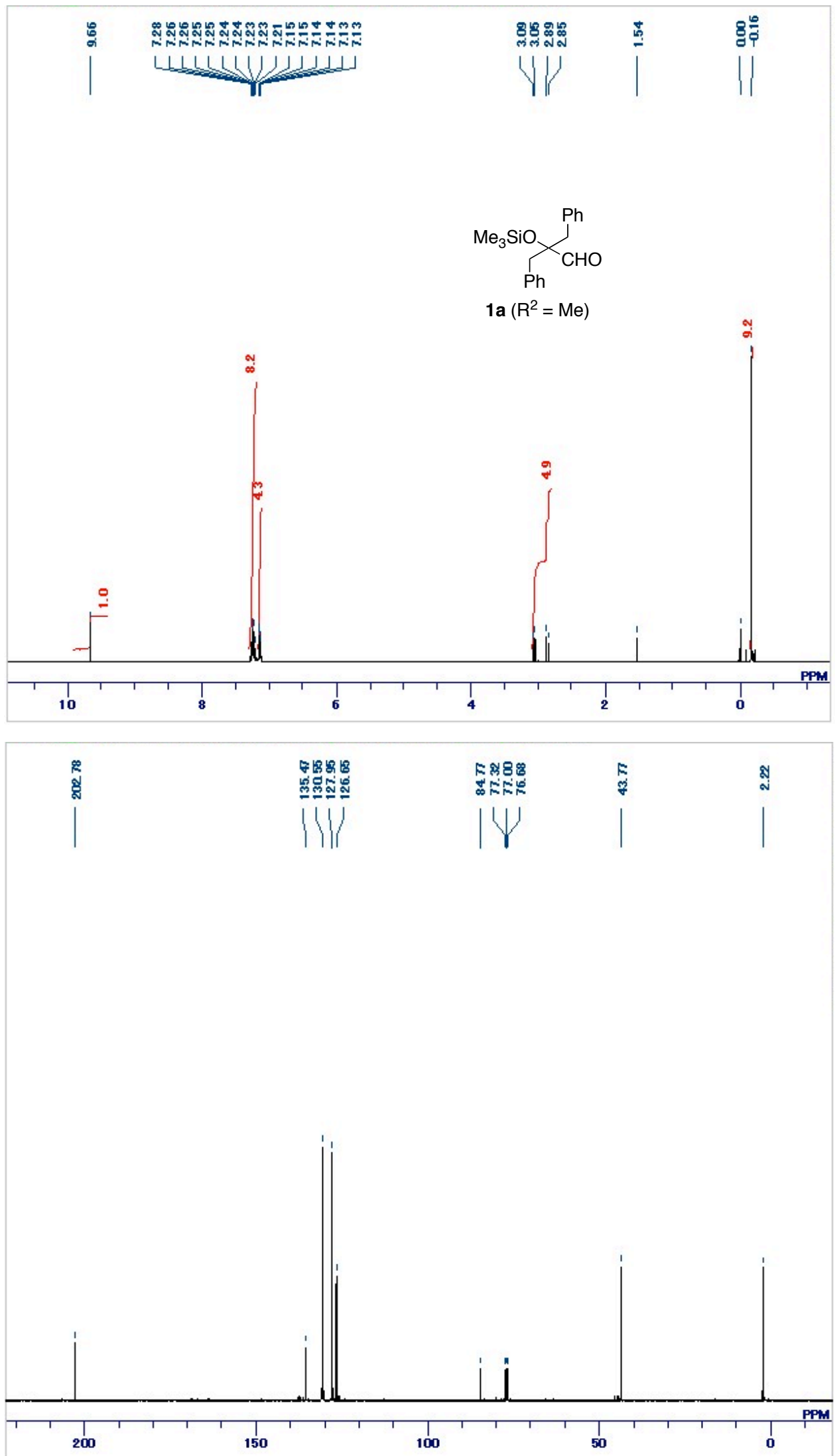




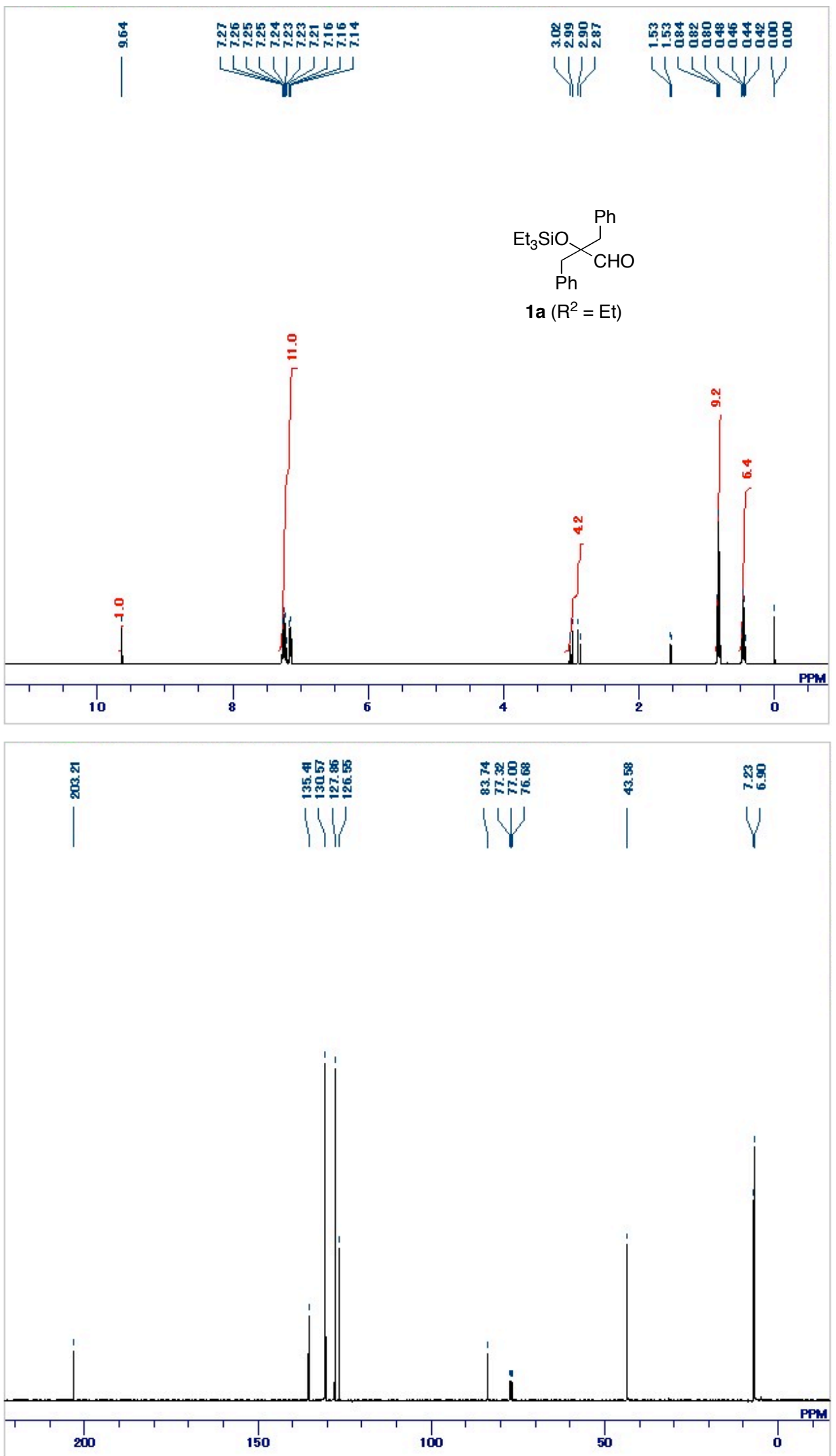




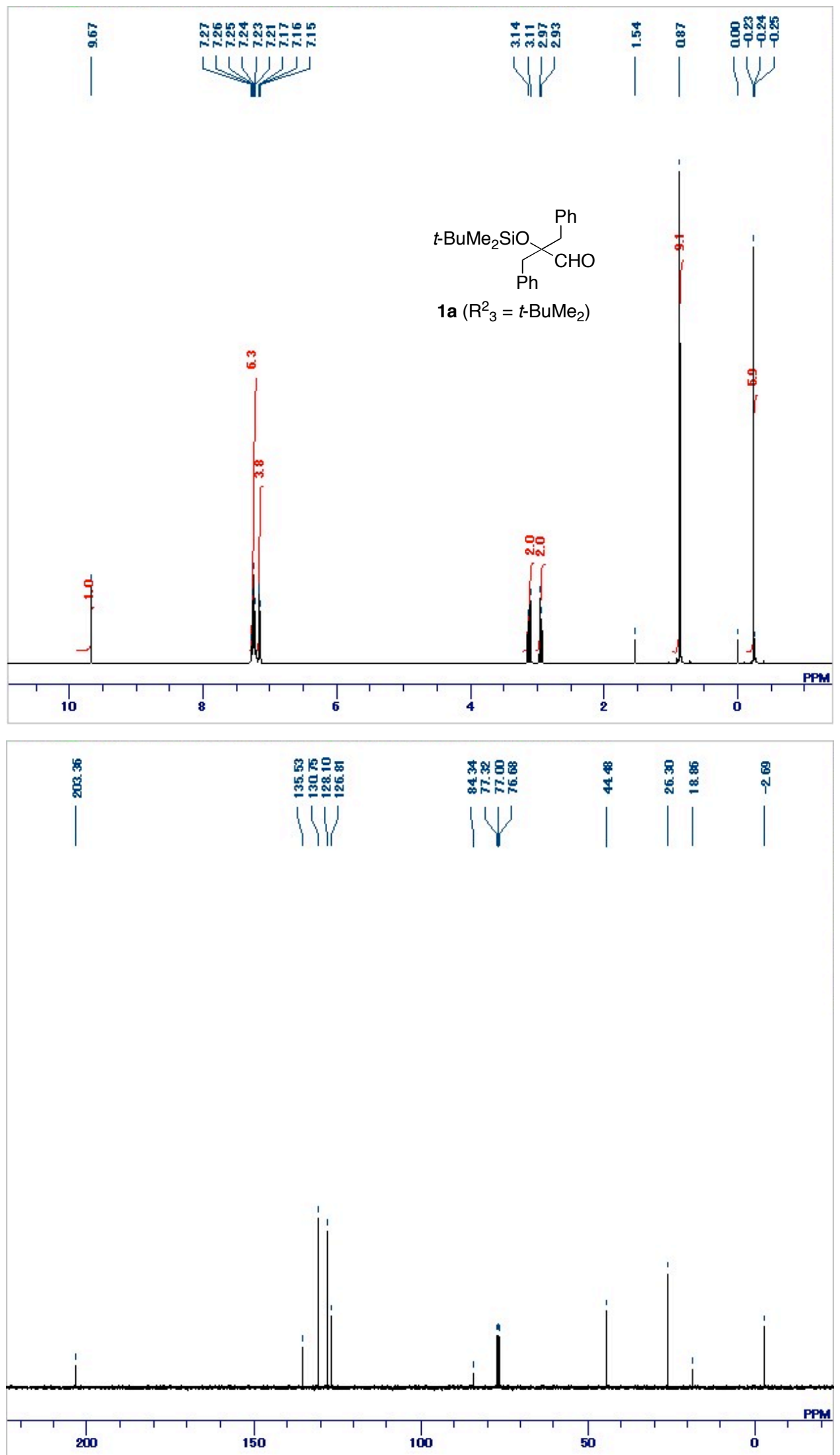




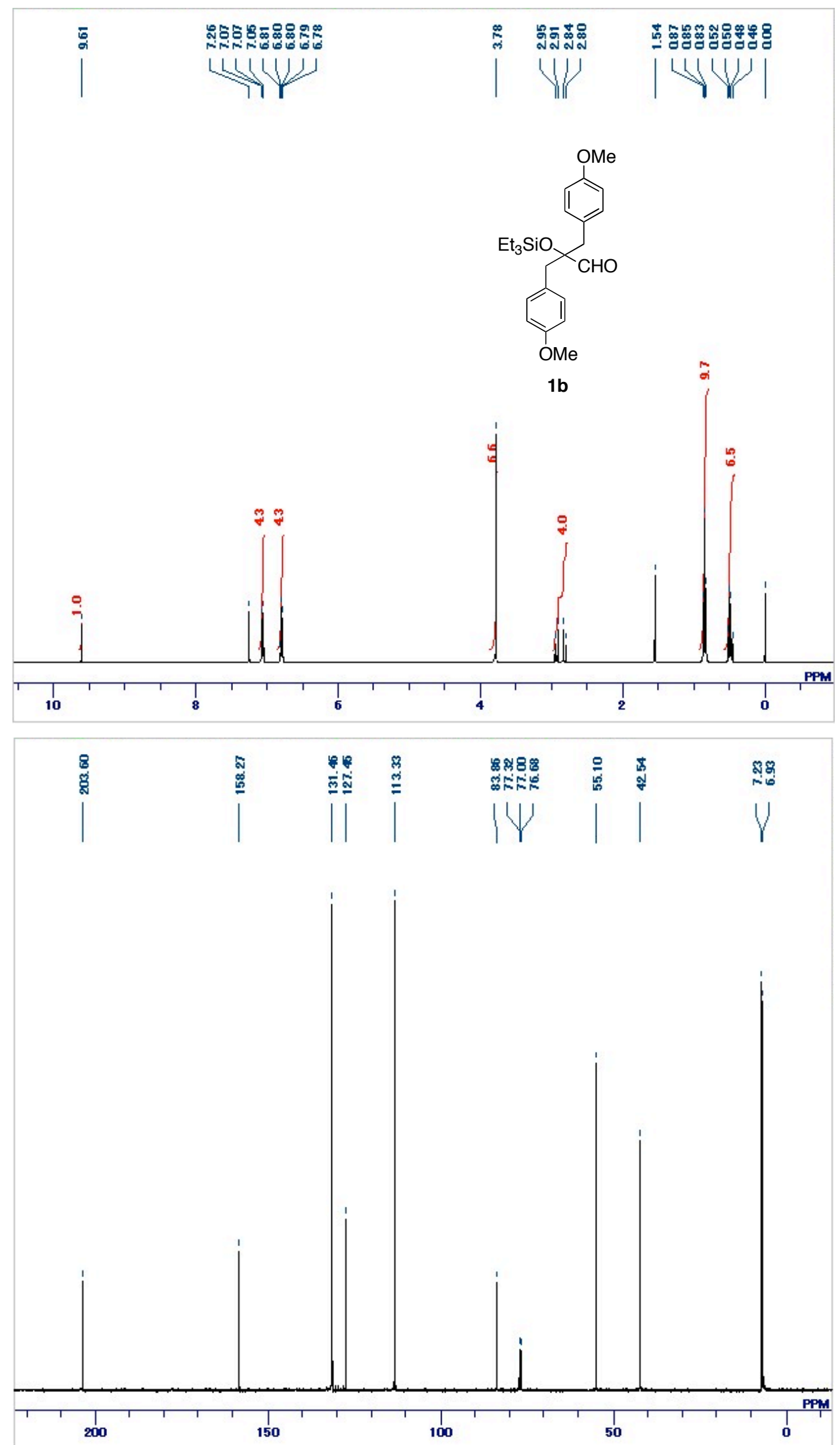




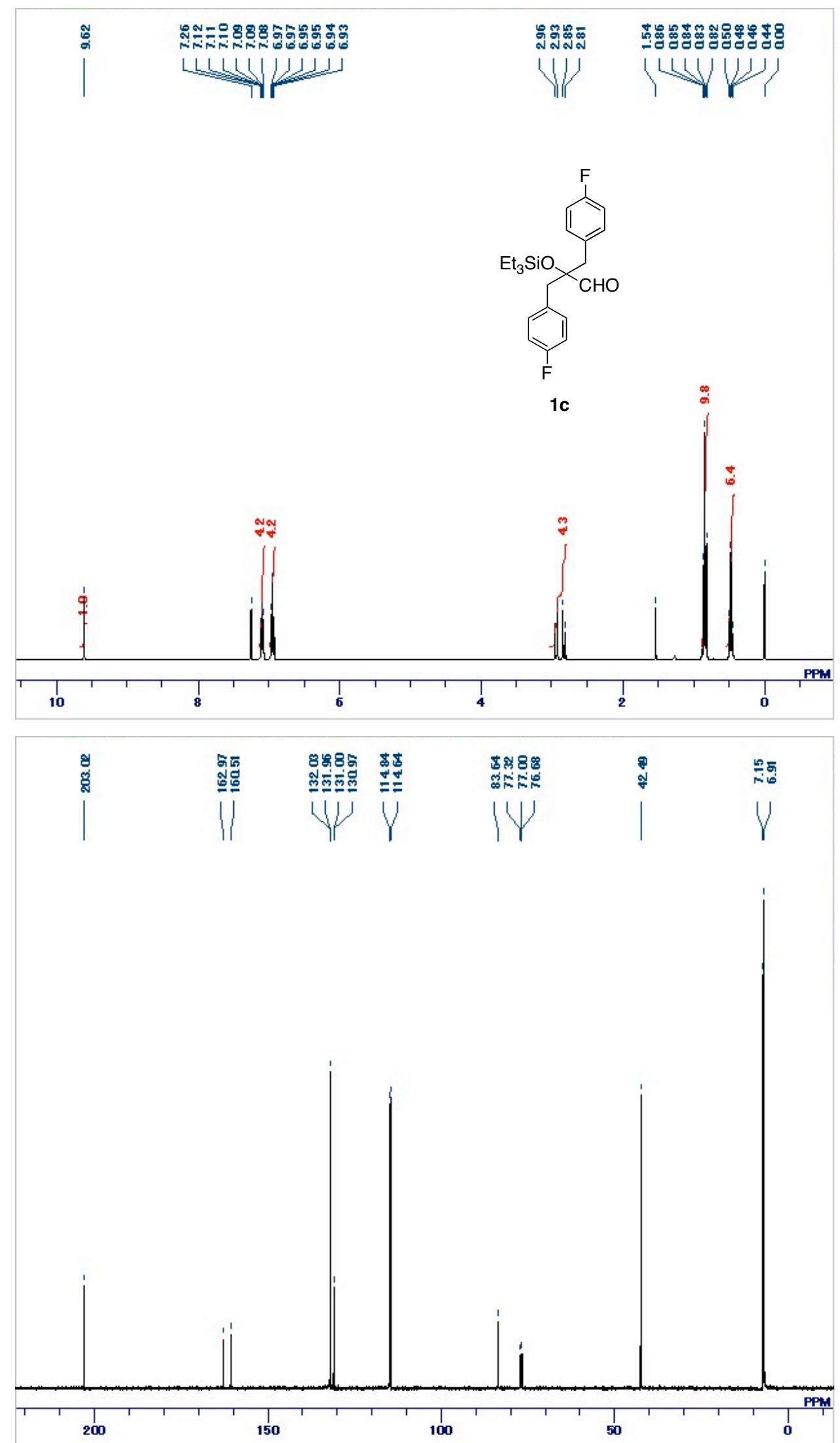




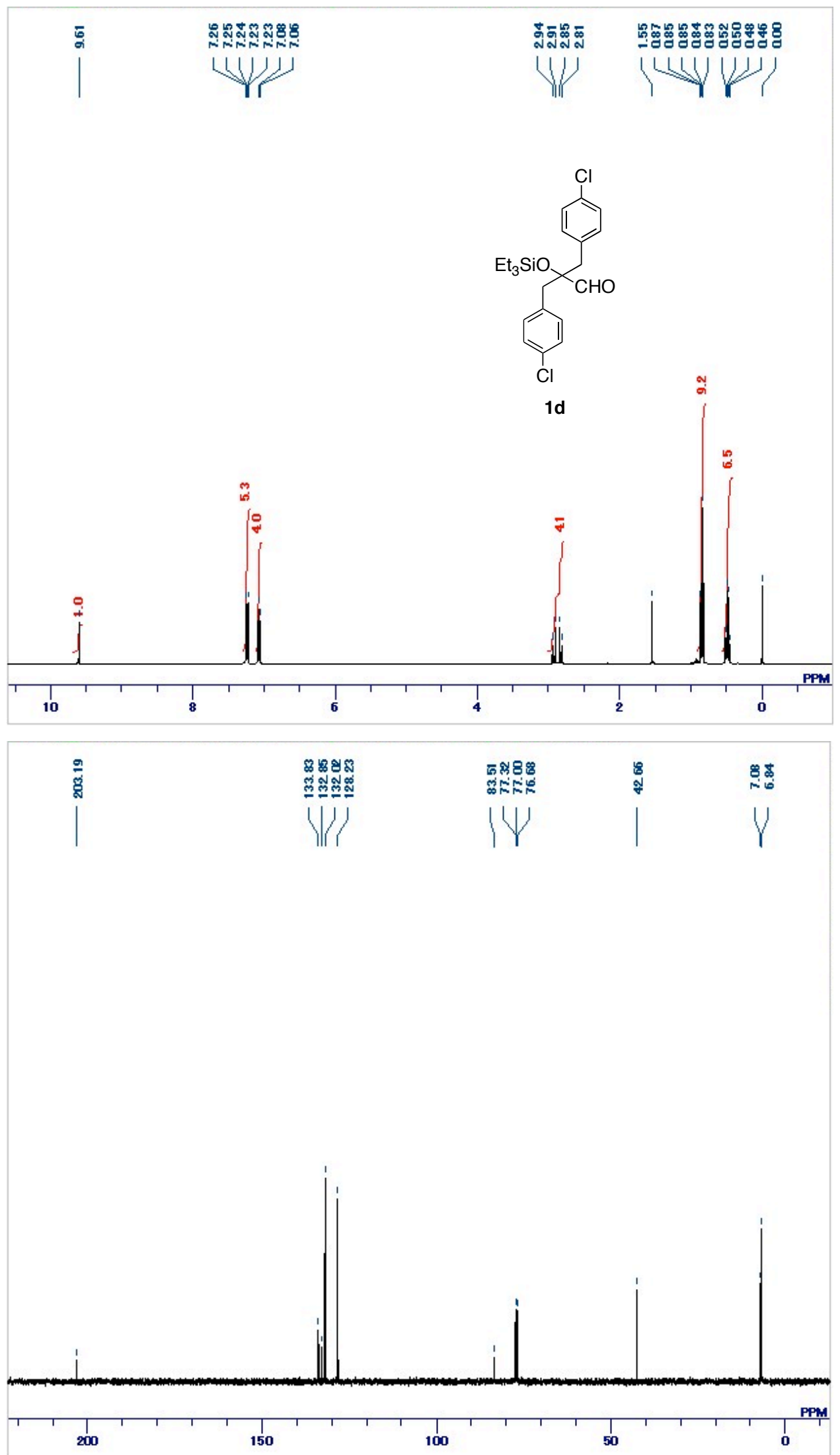




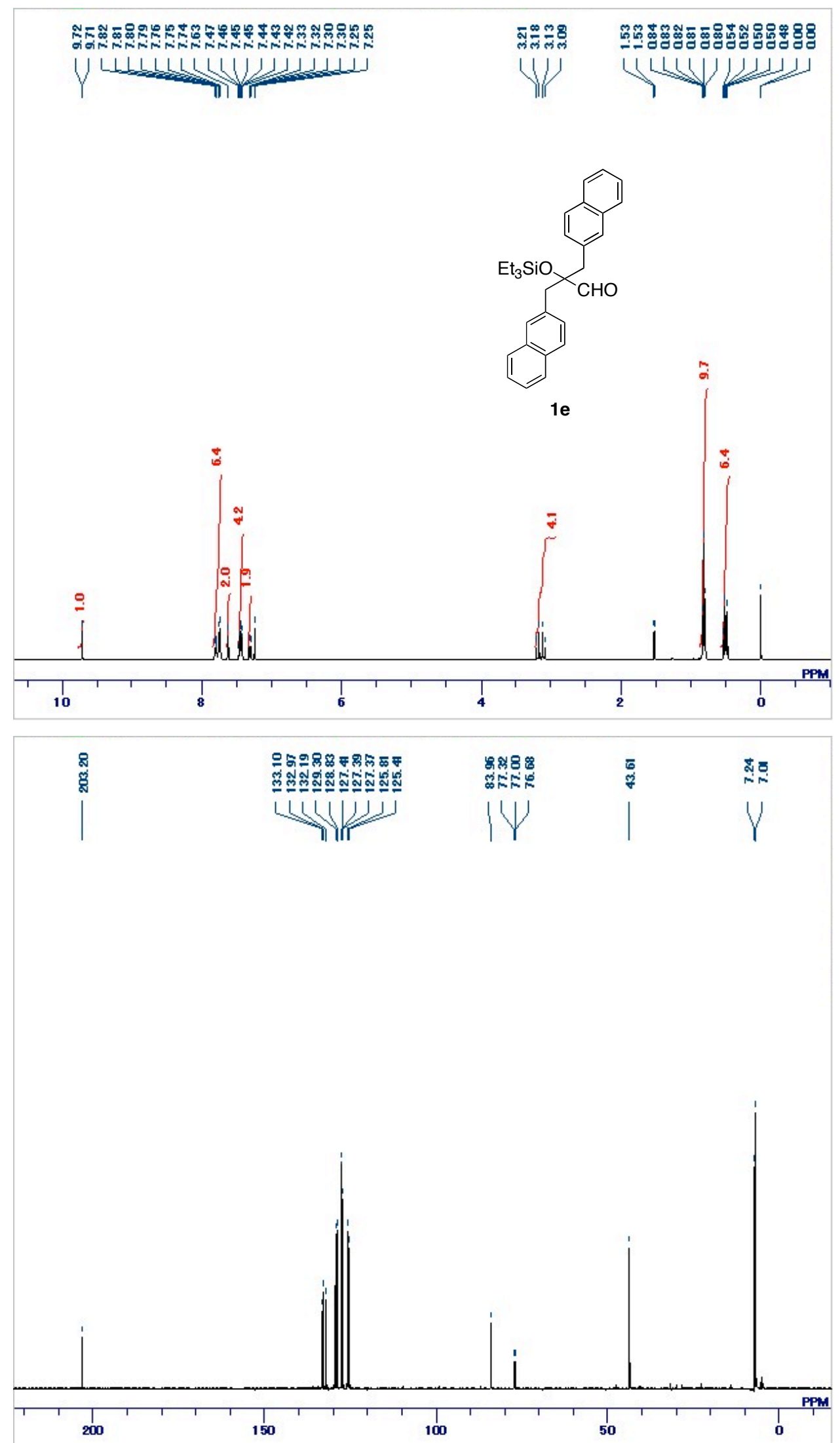




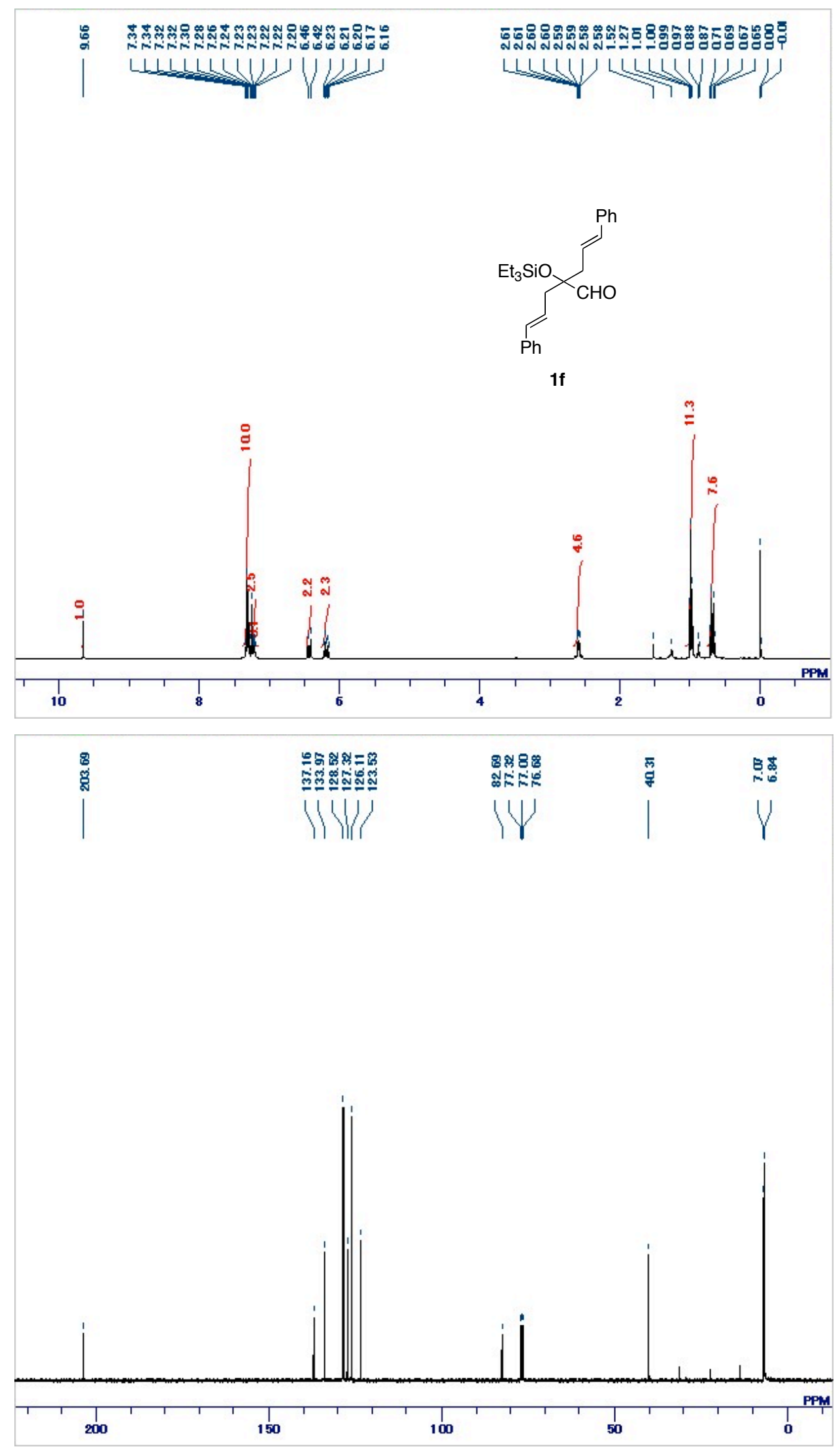




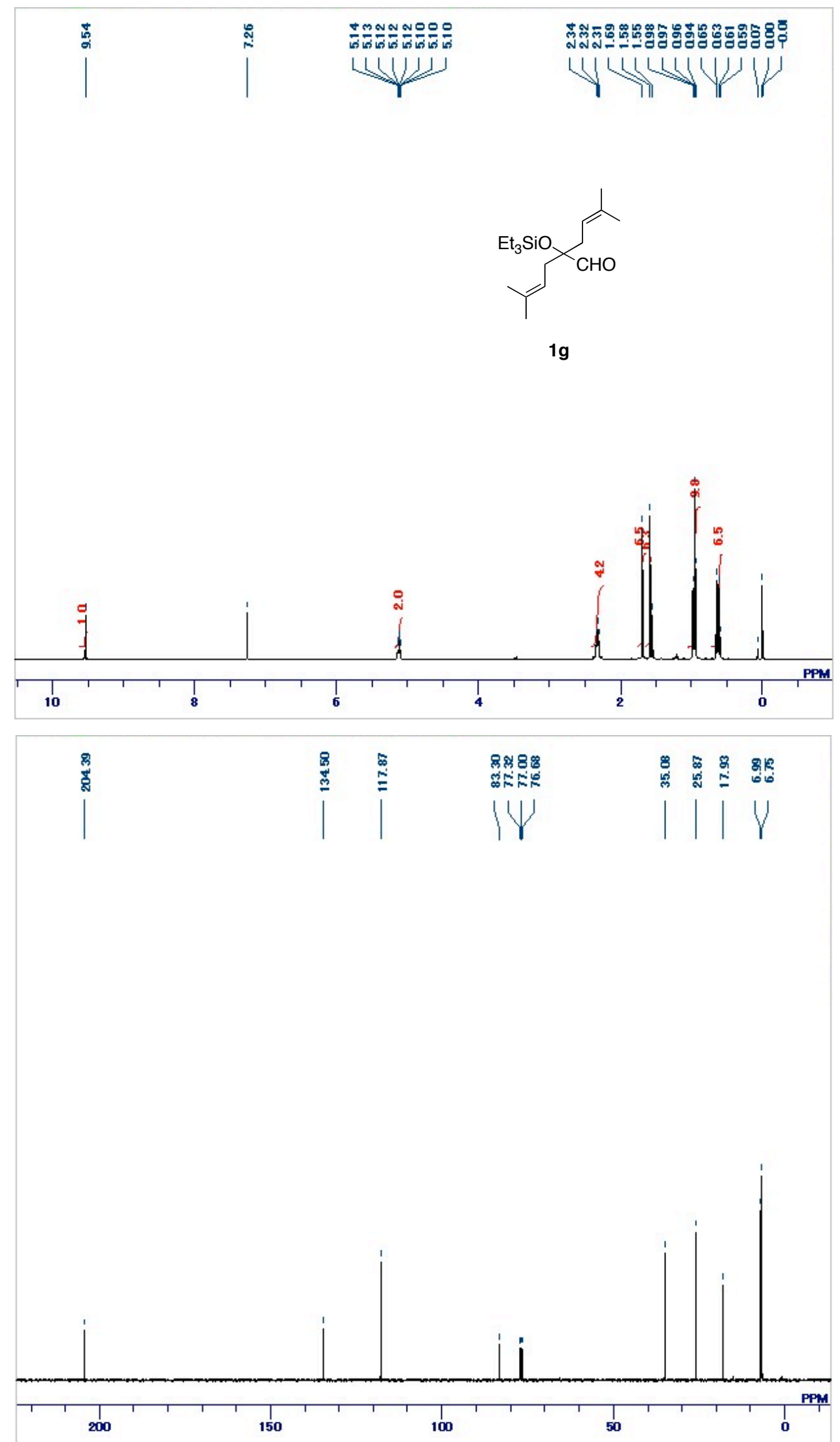




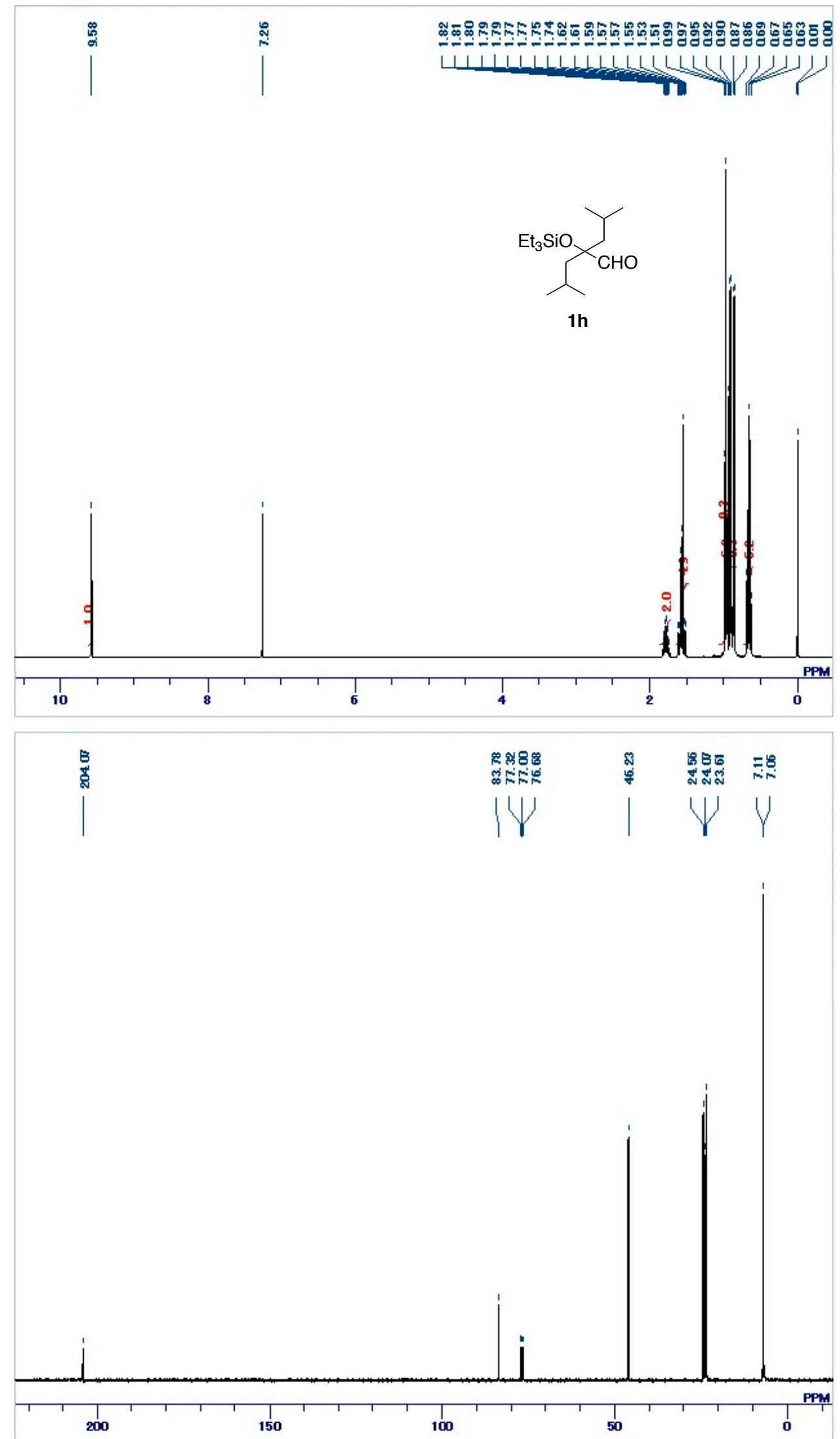




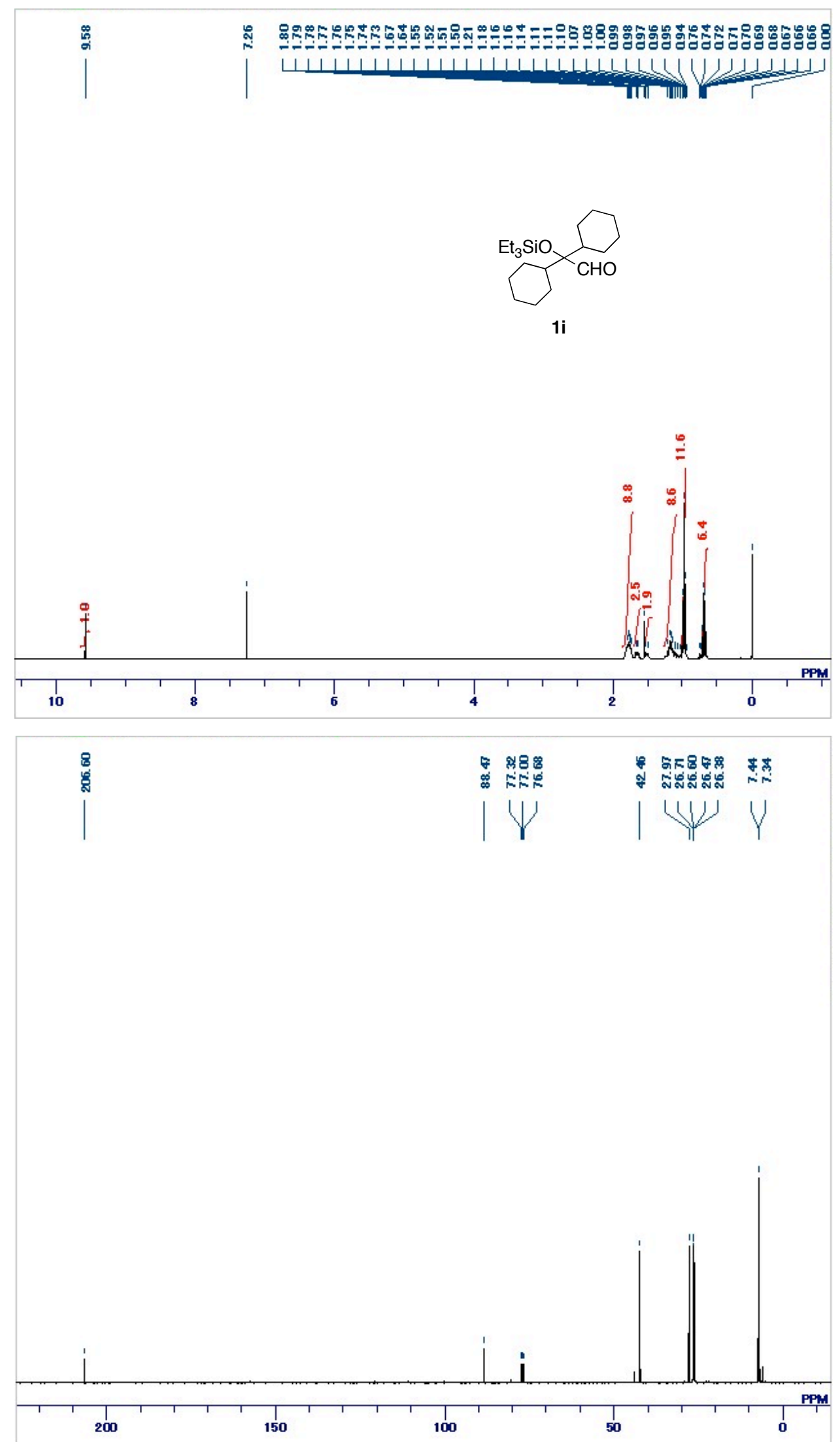




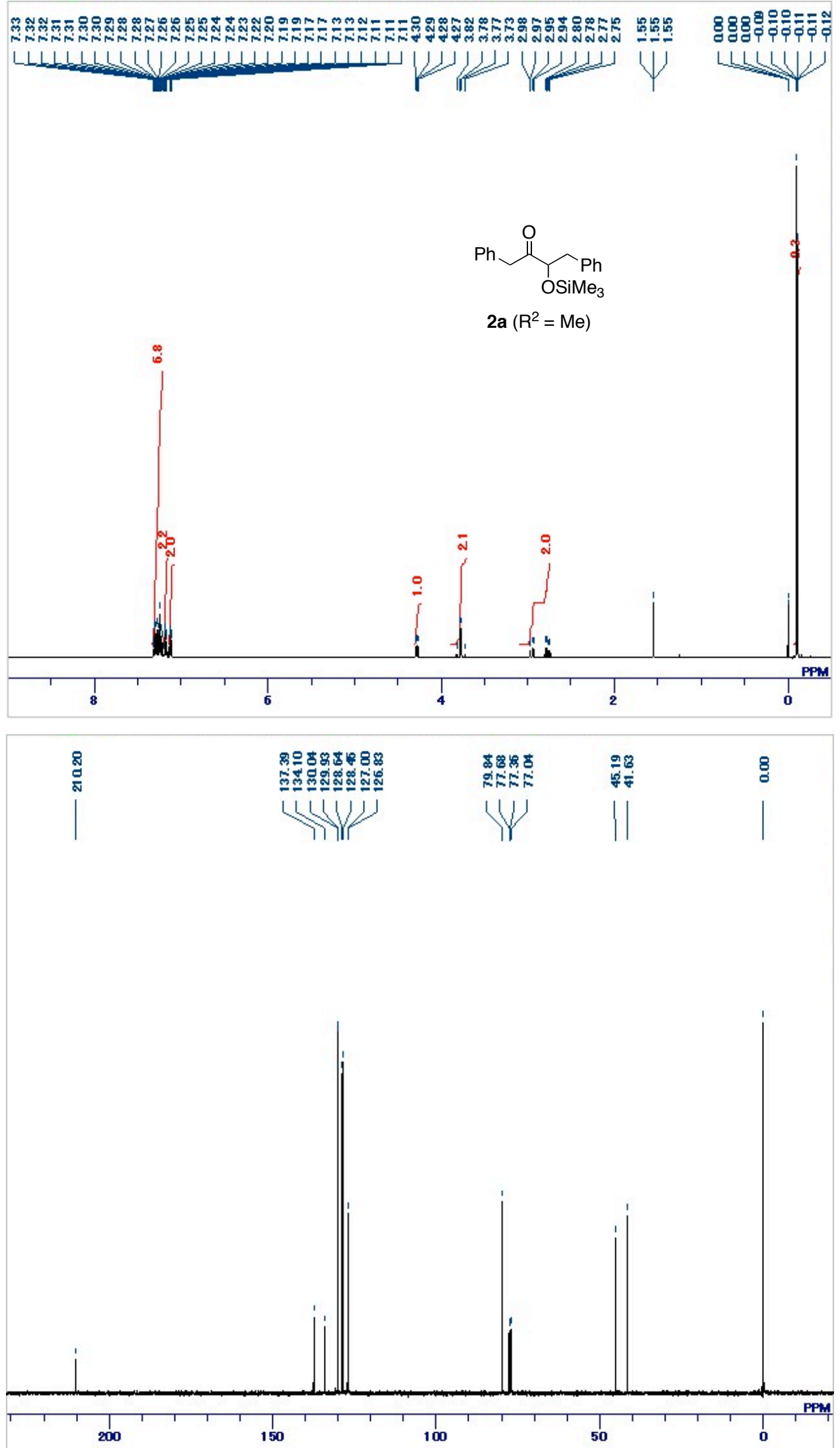




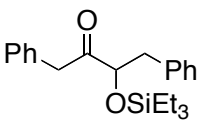

2a $\left(R^{2}=E t\right)$
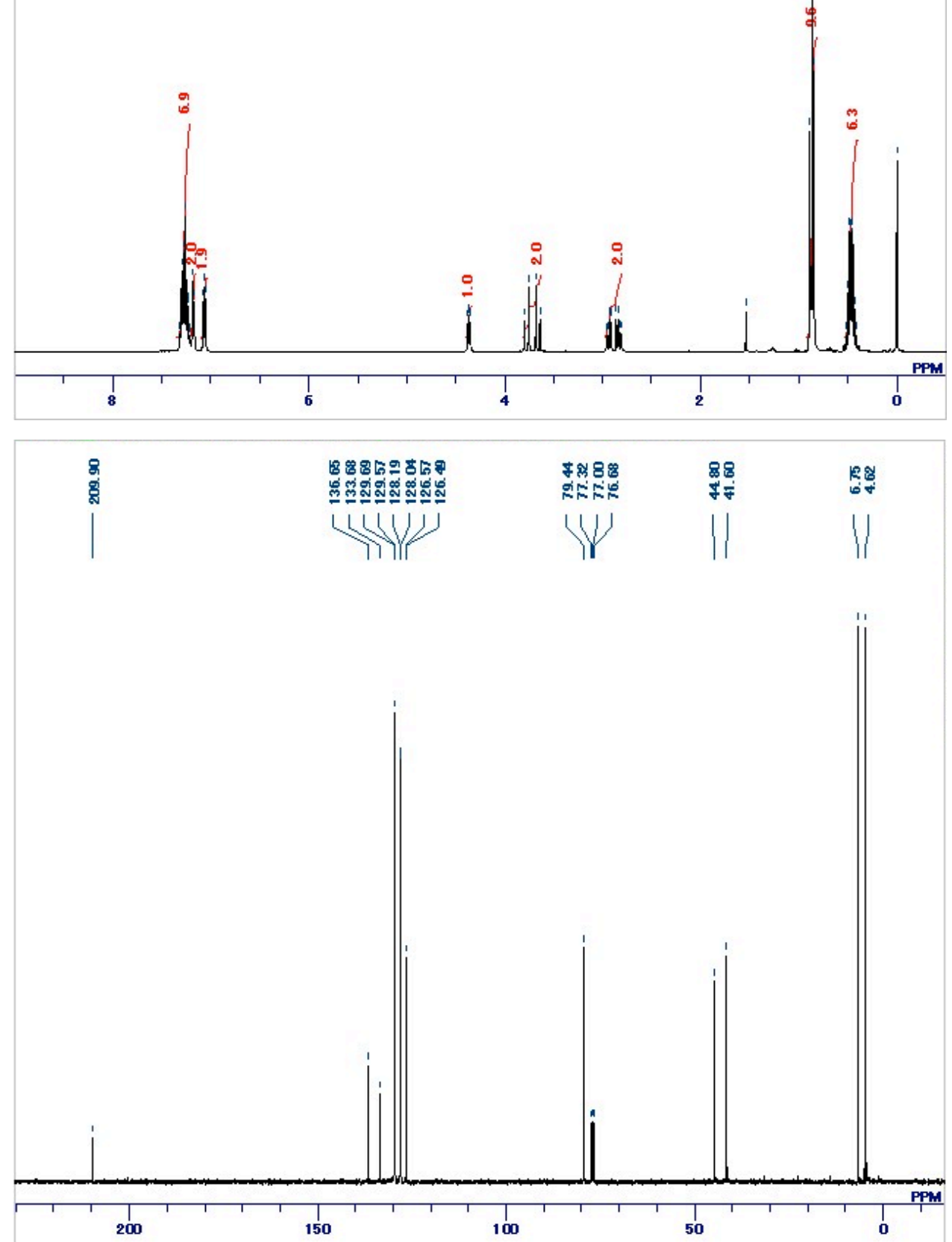


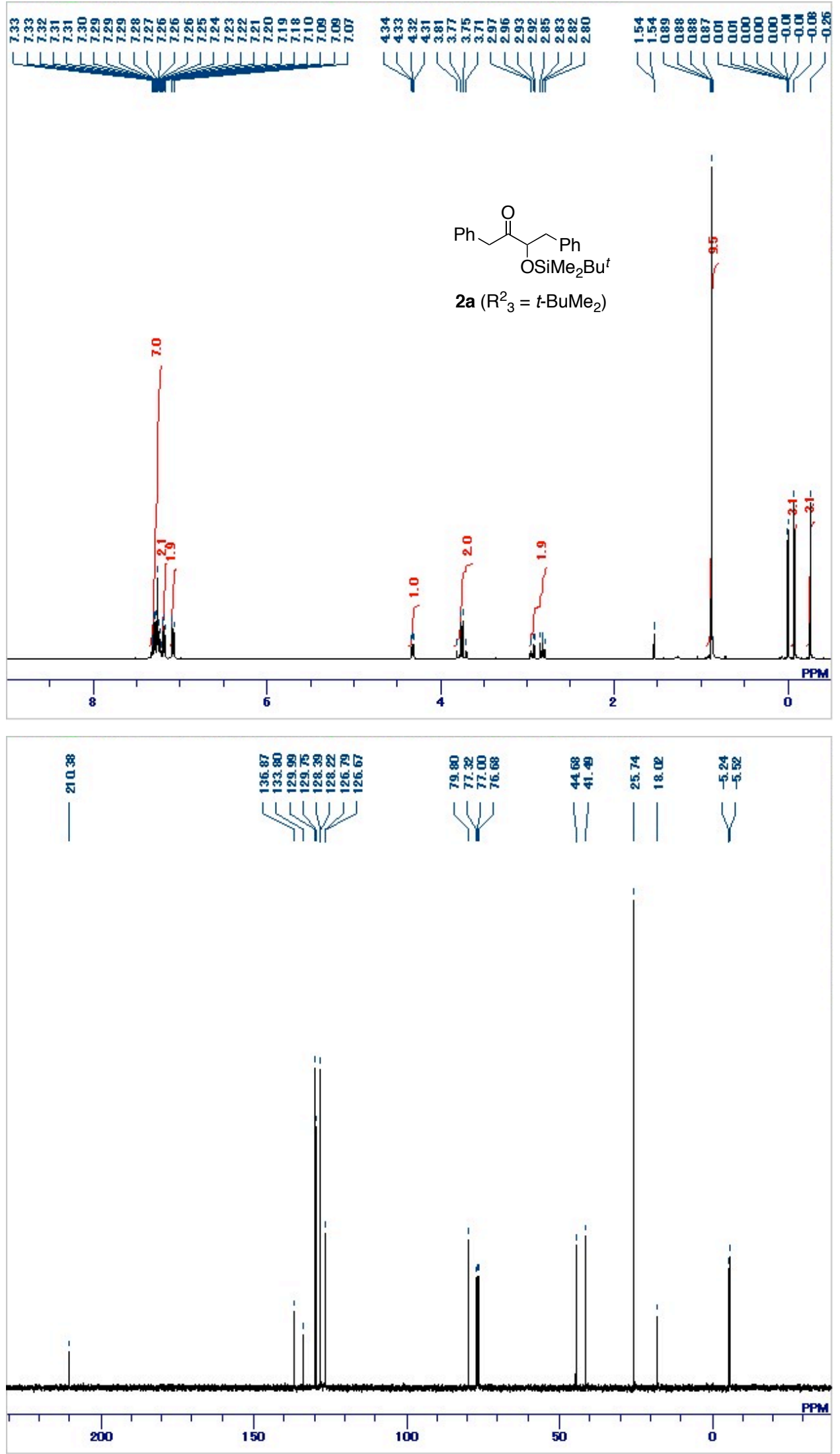




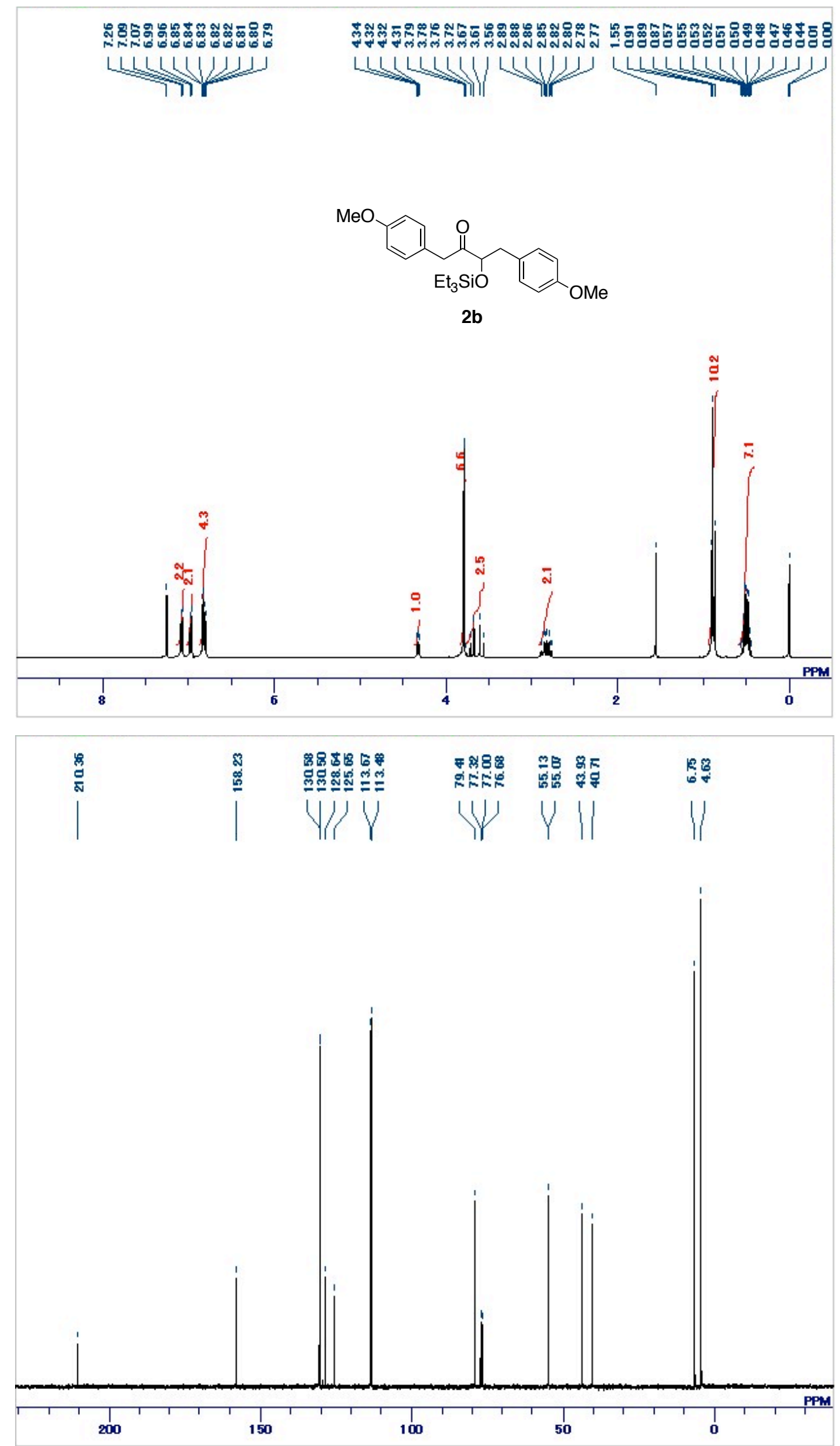




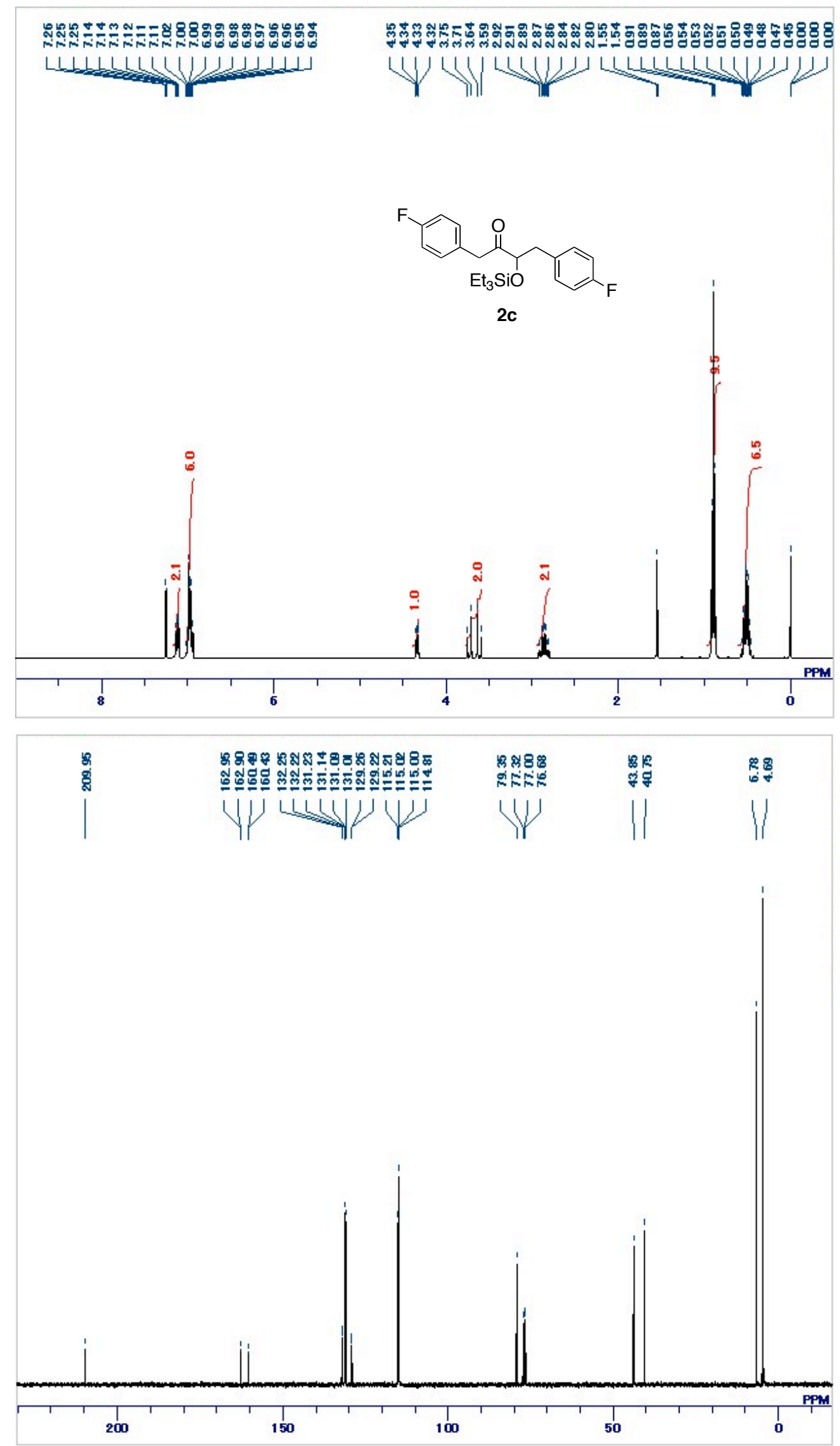




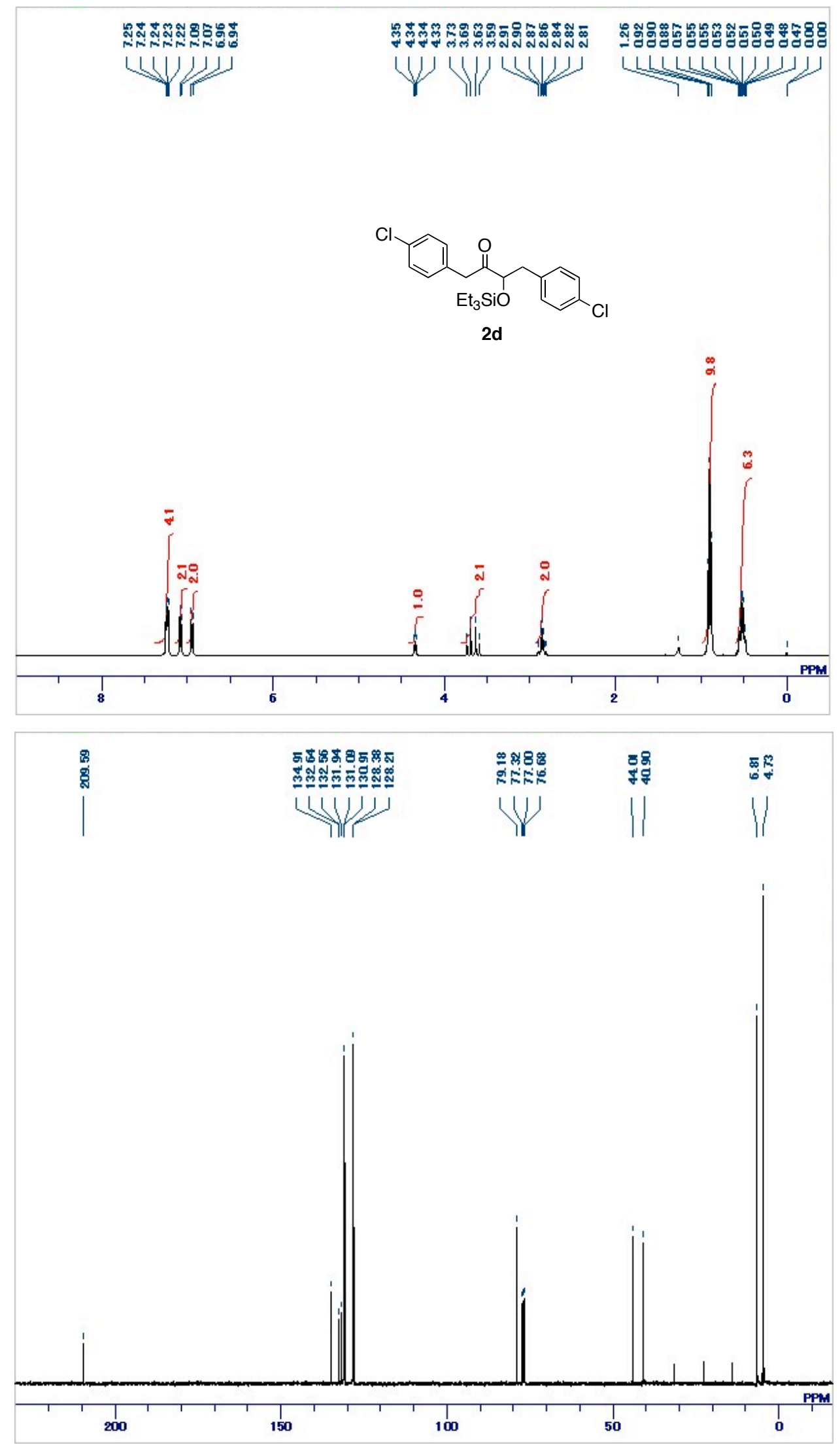




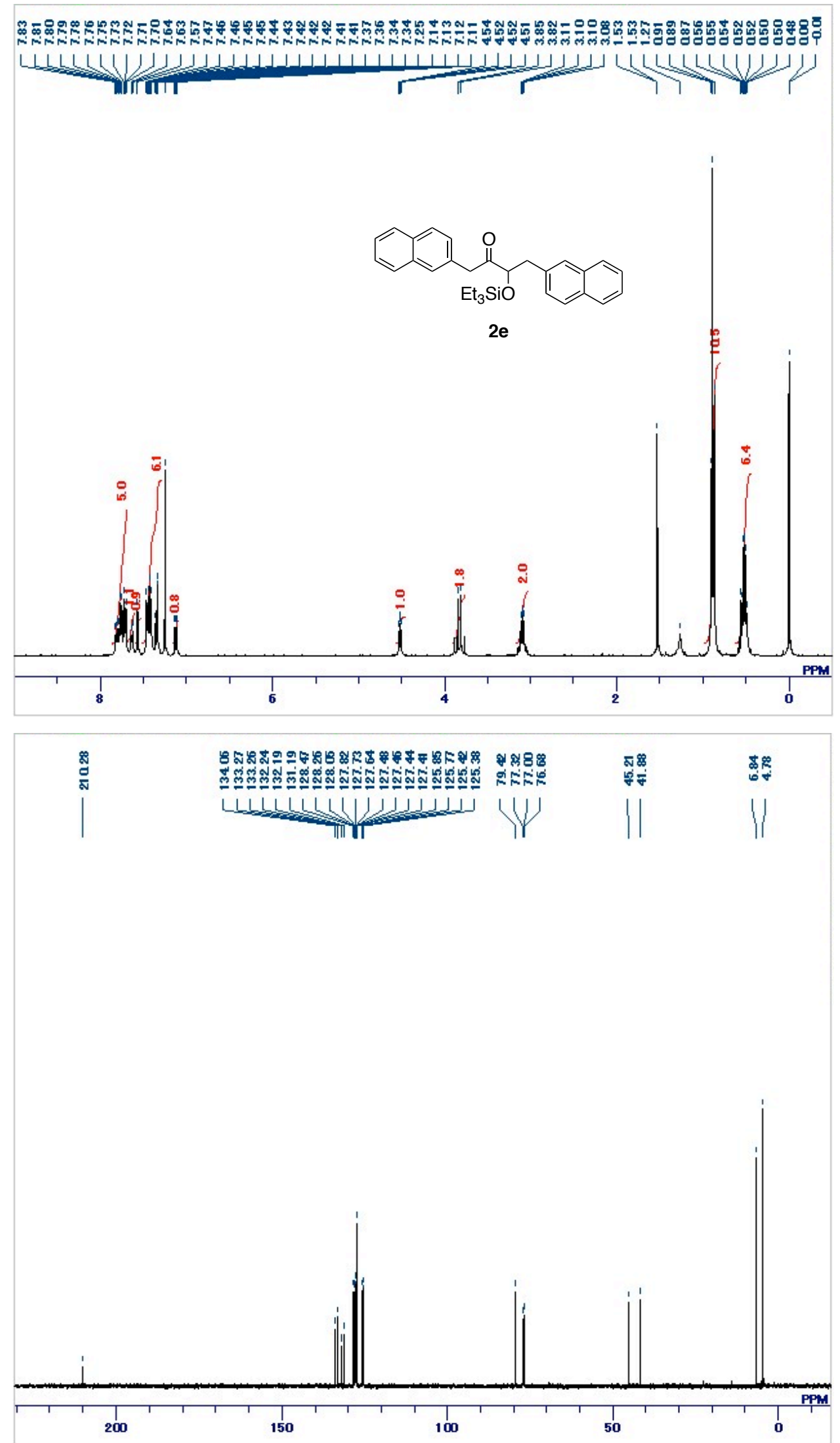



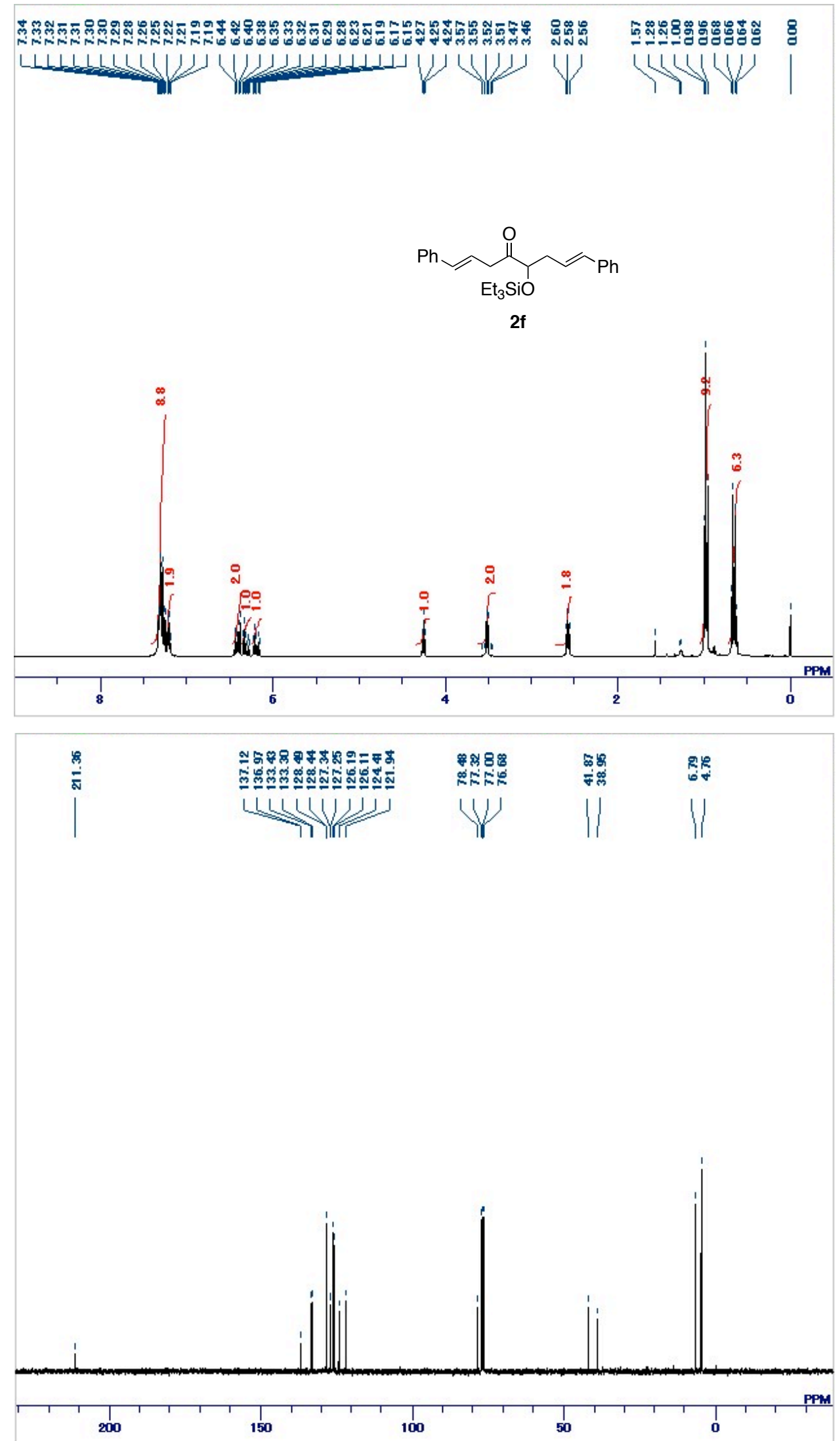


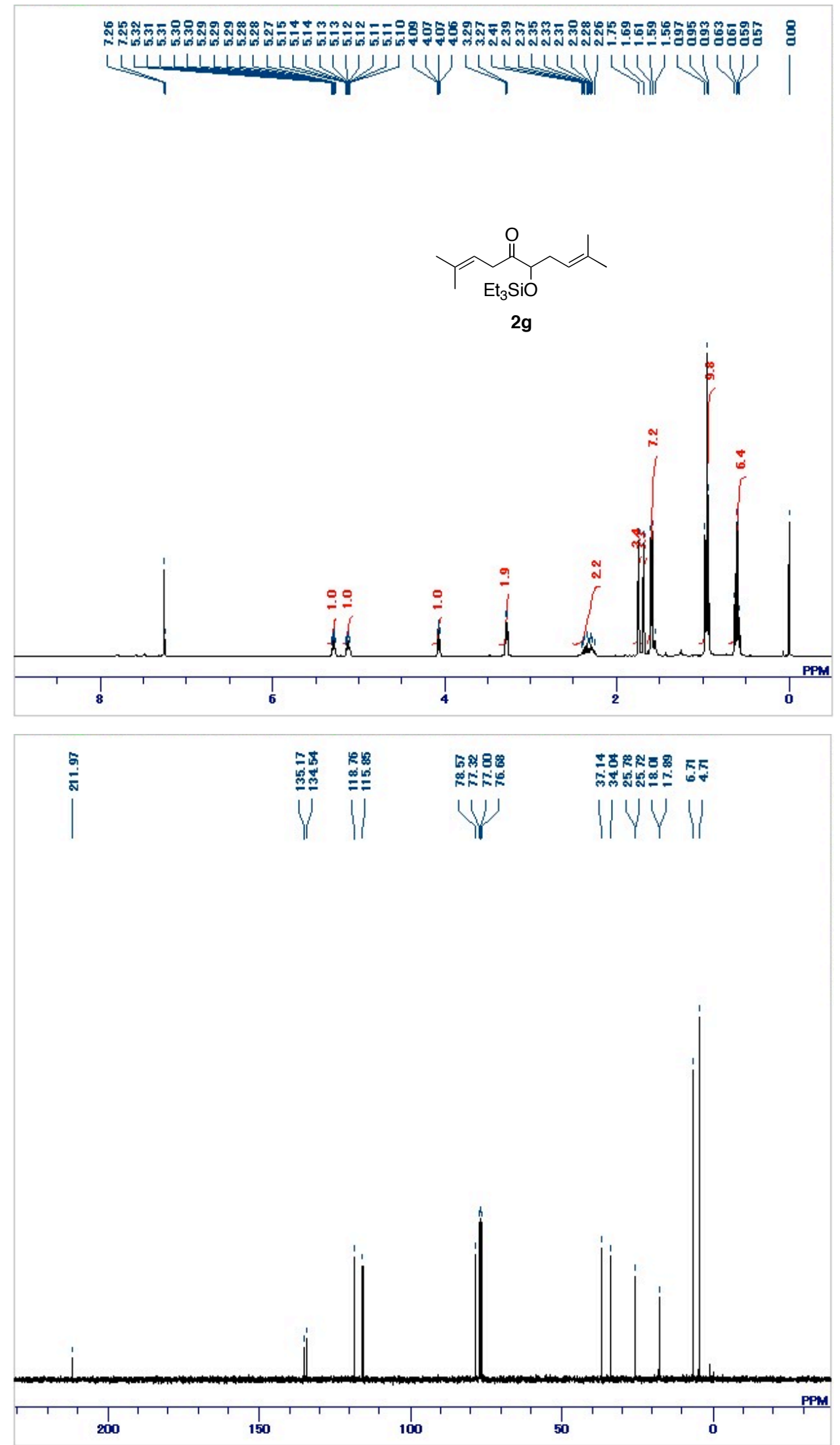




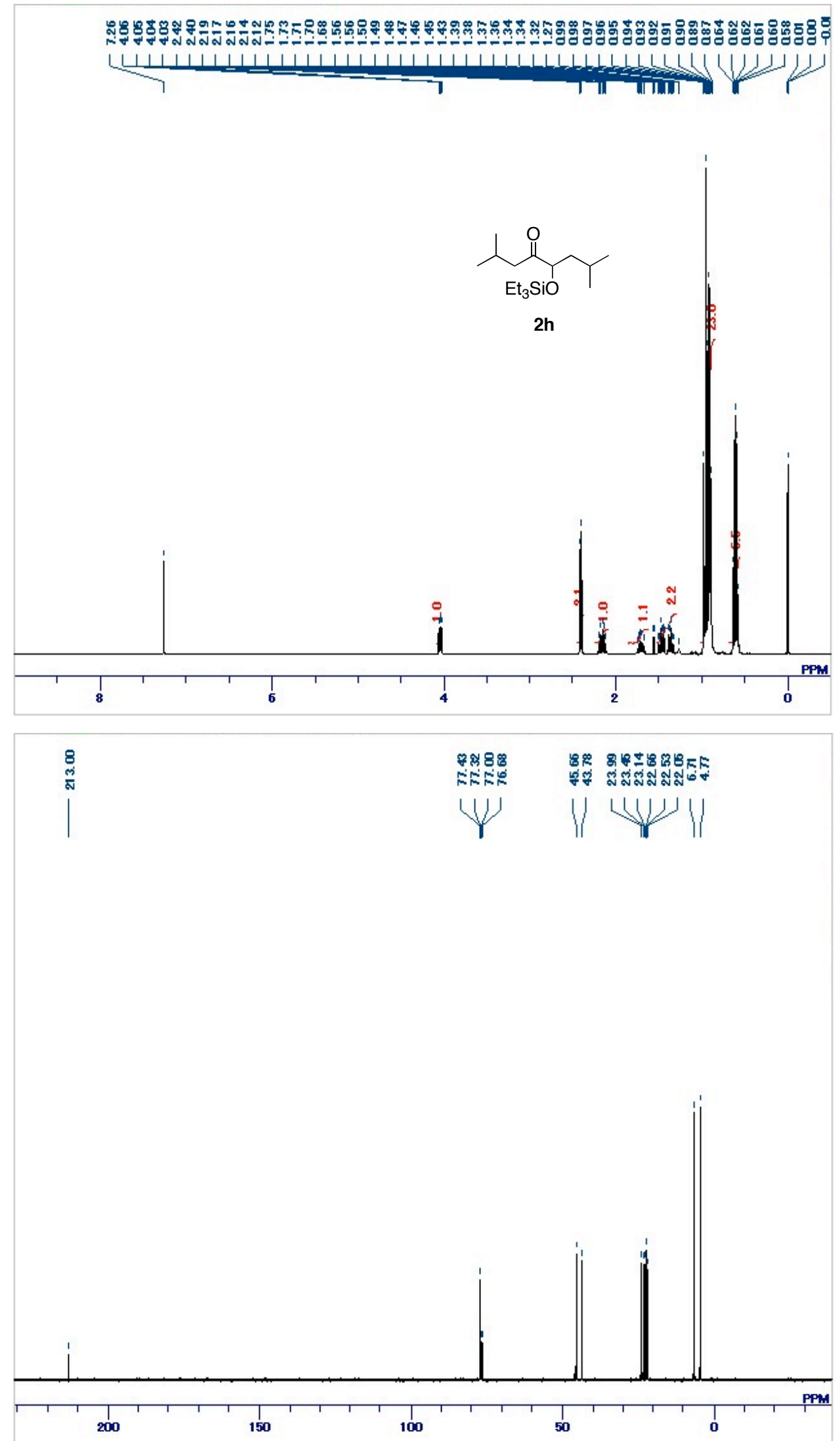




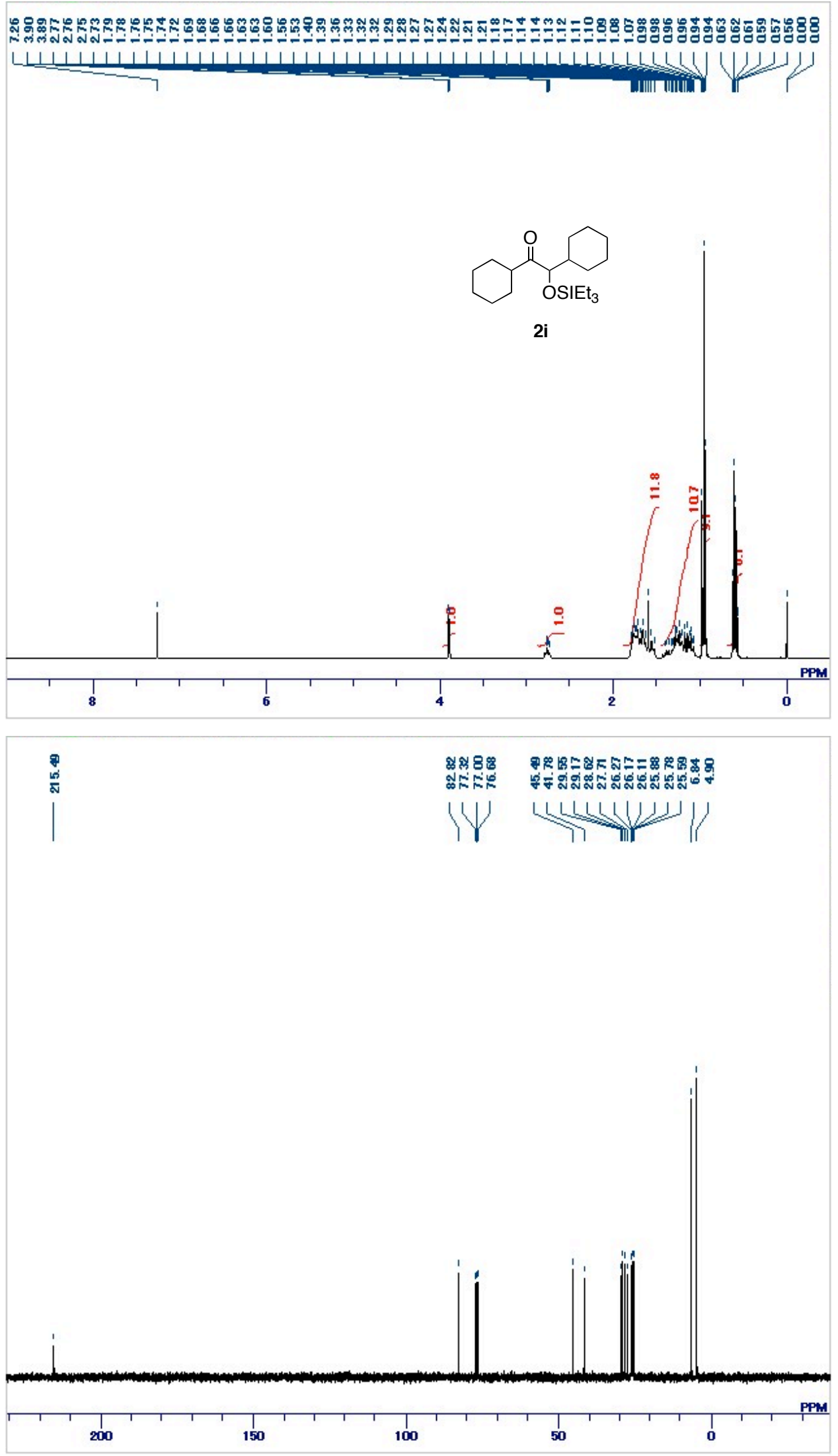




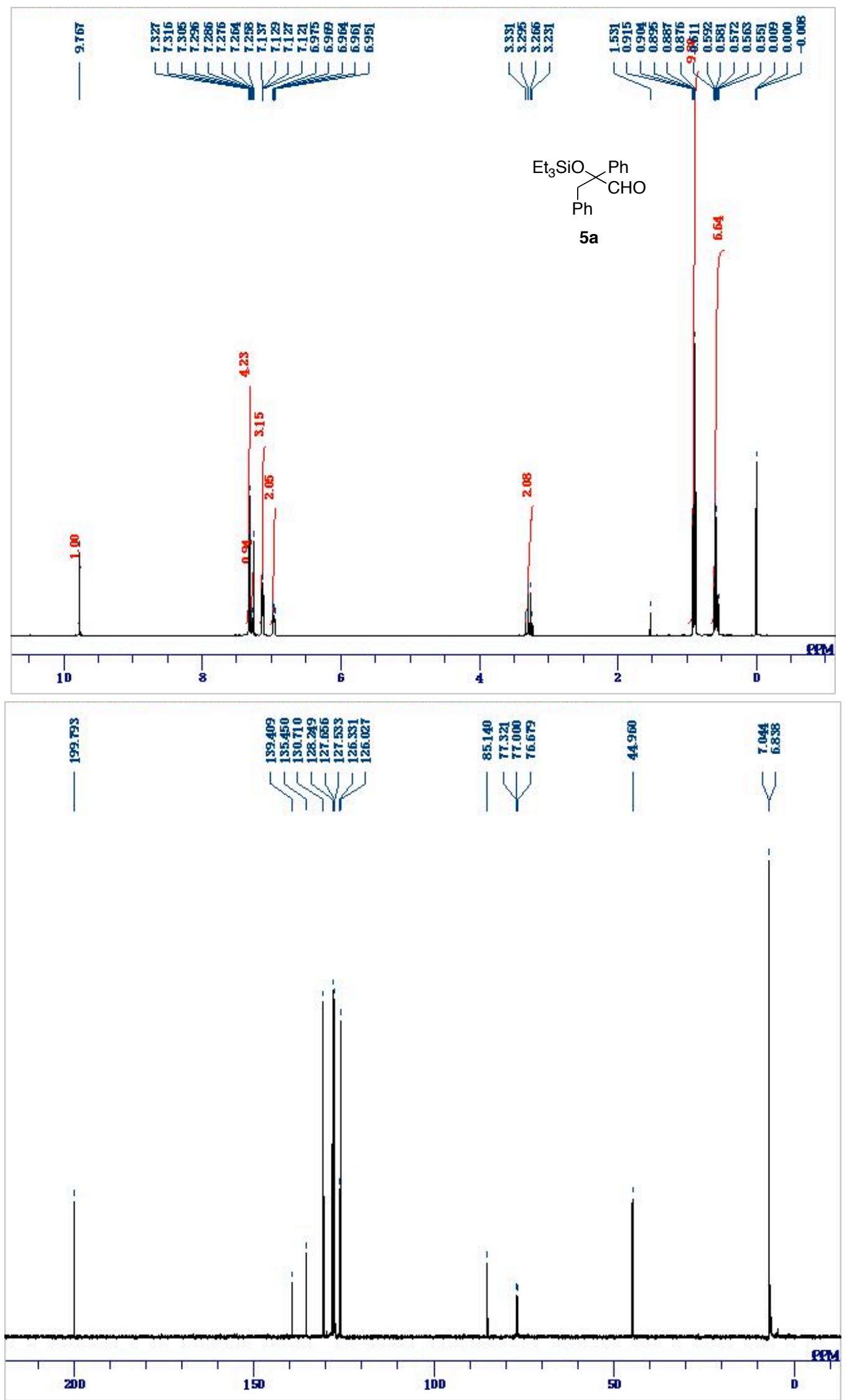




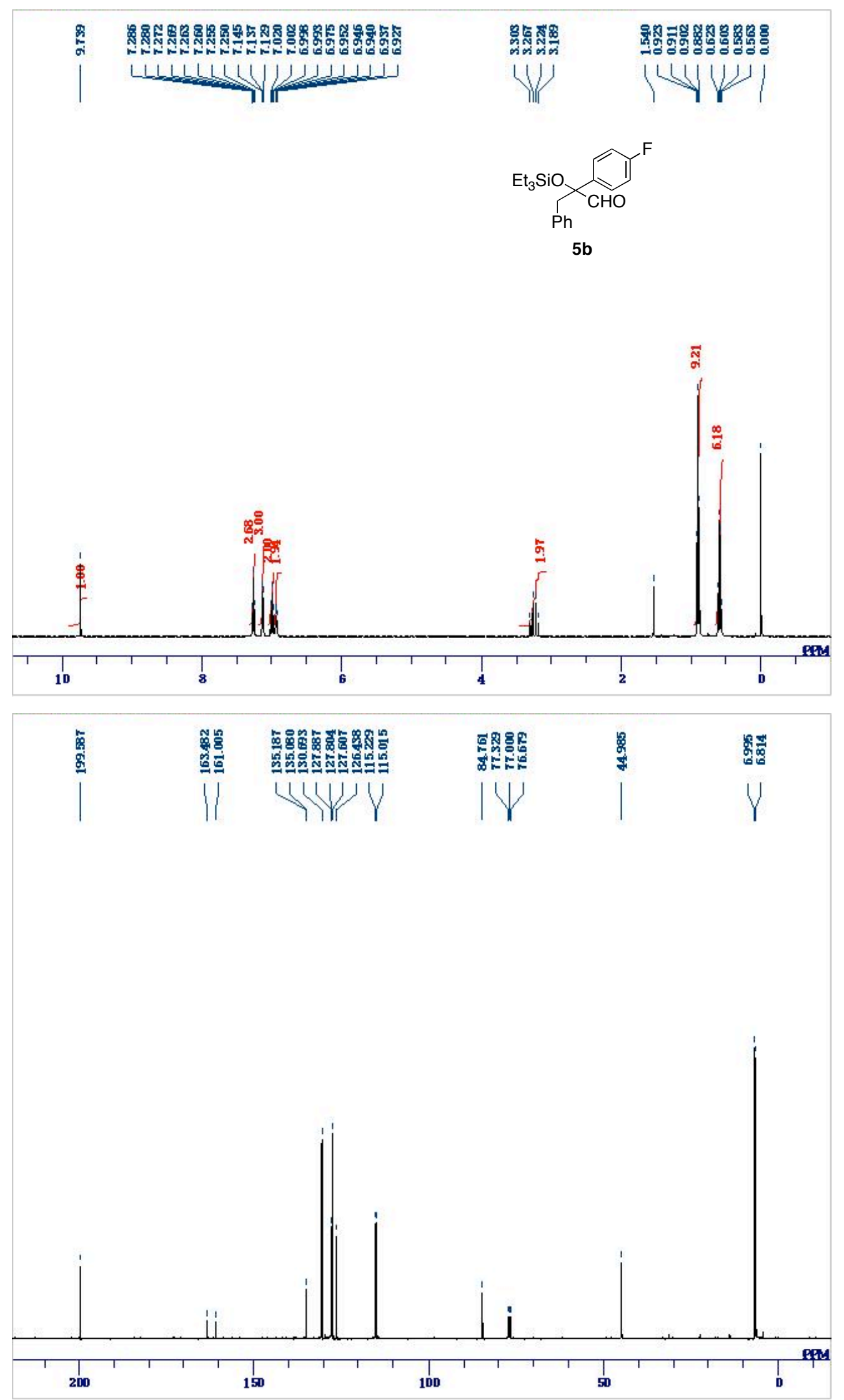




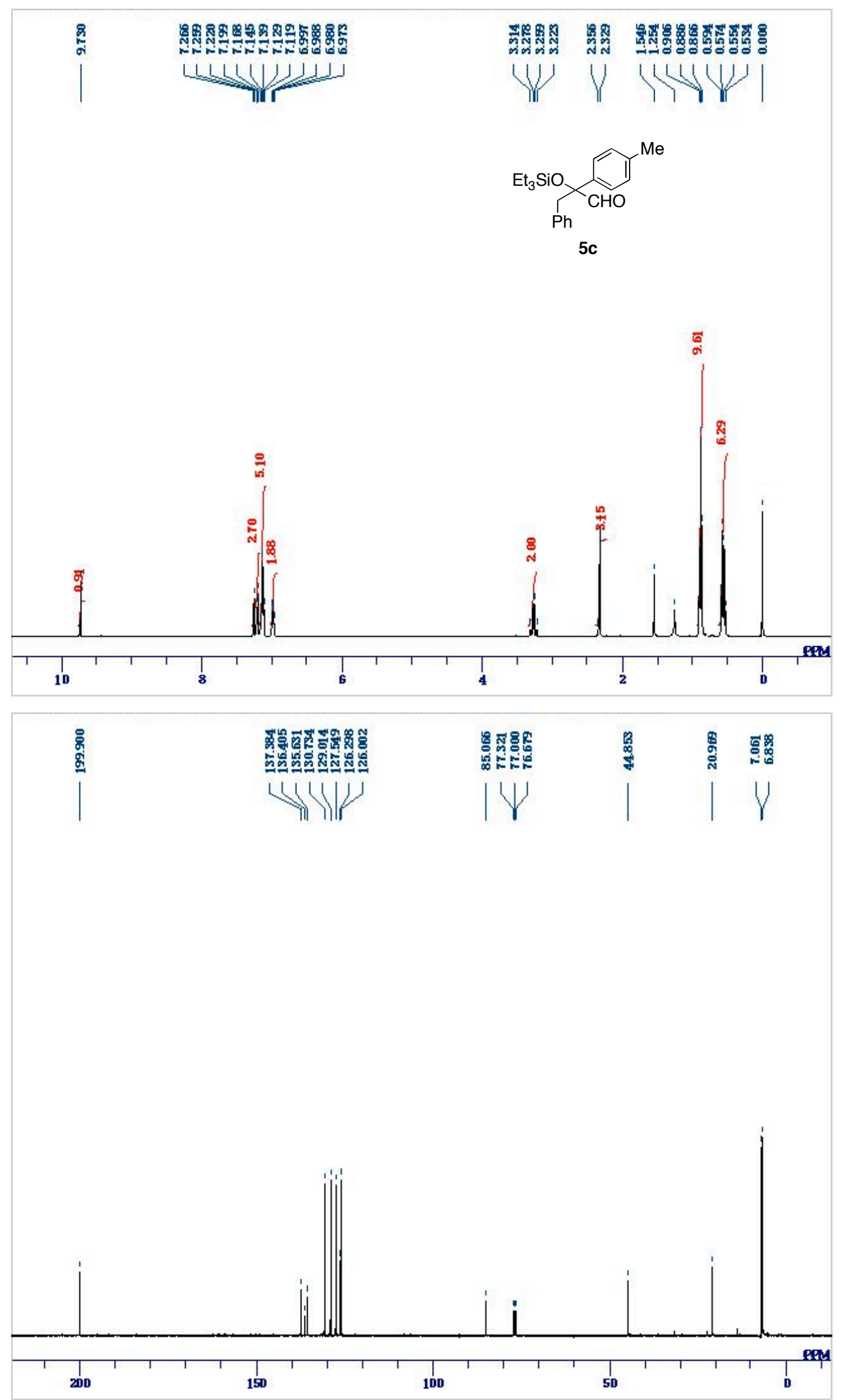




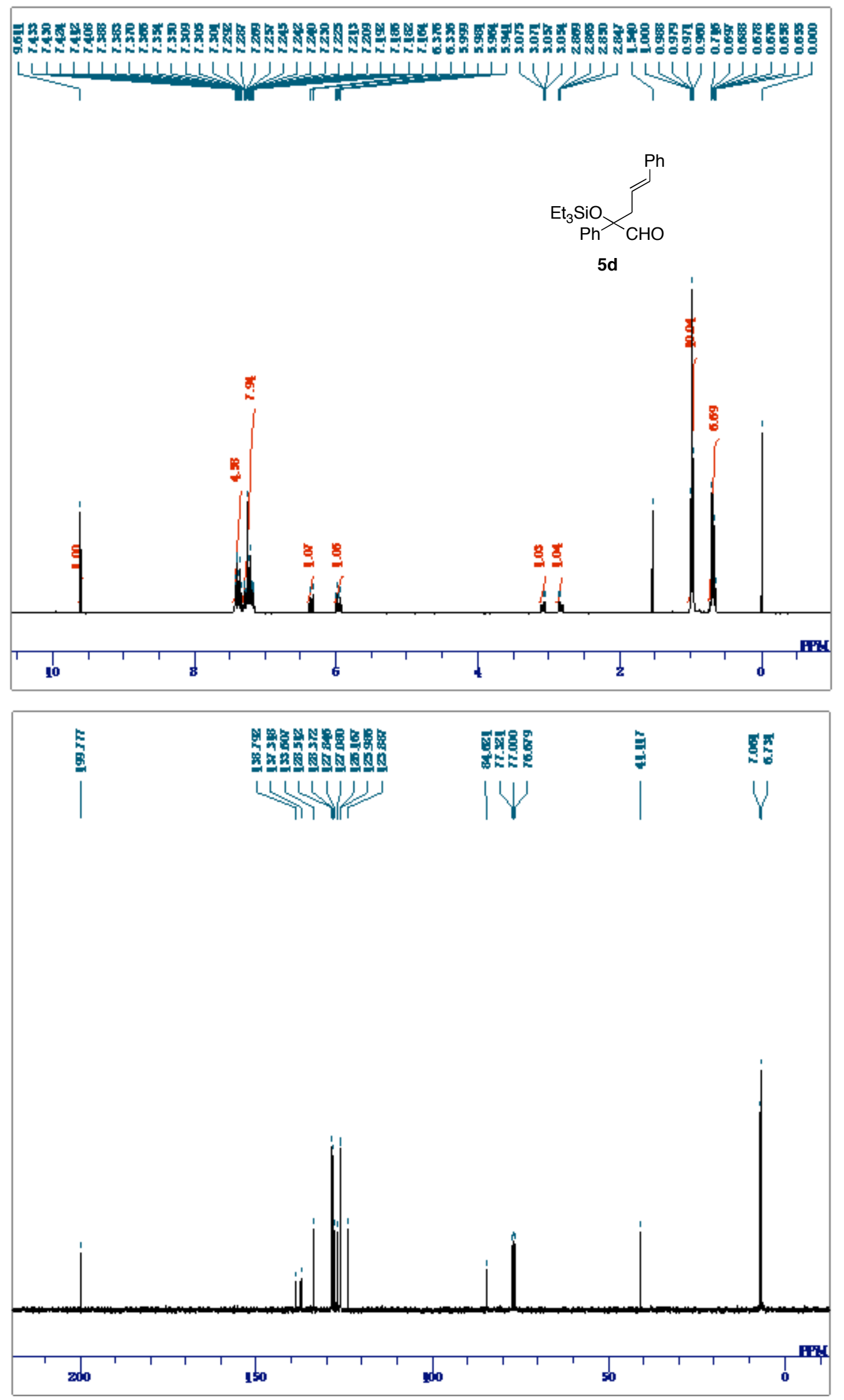




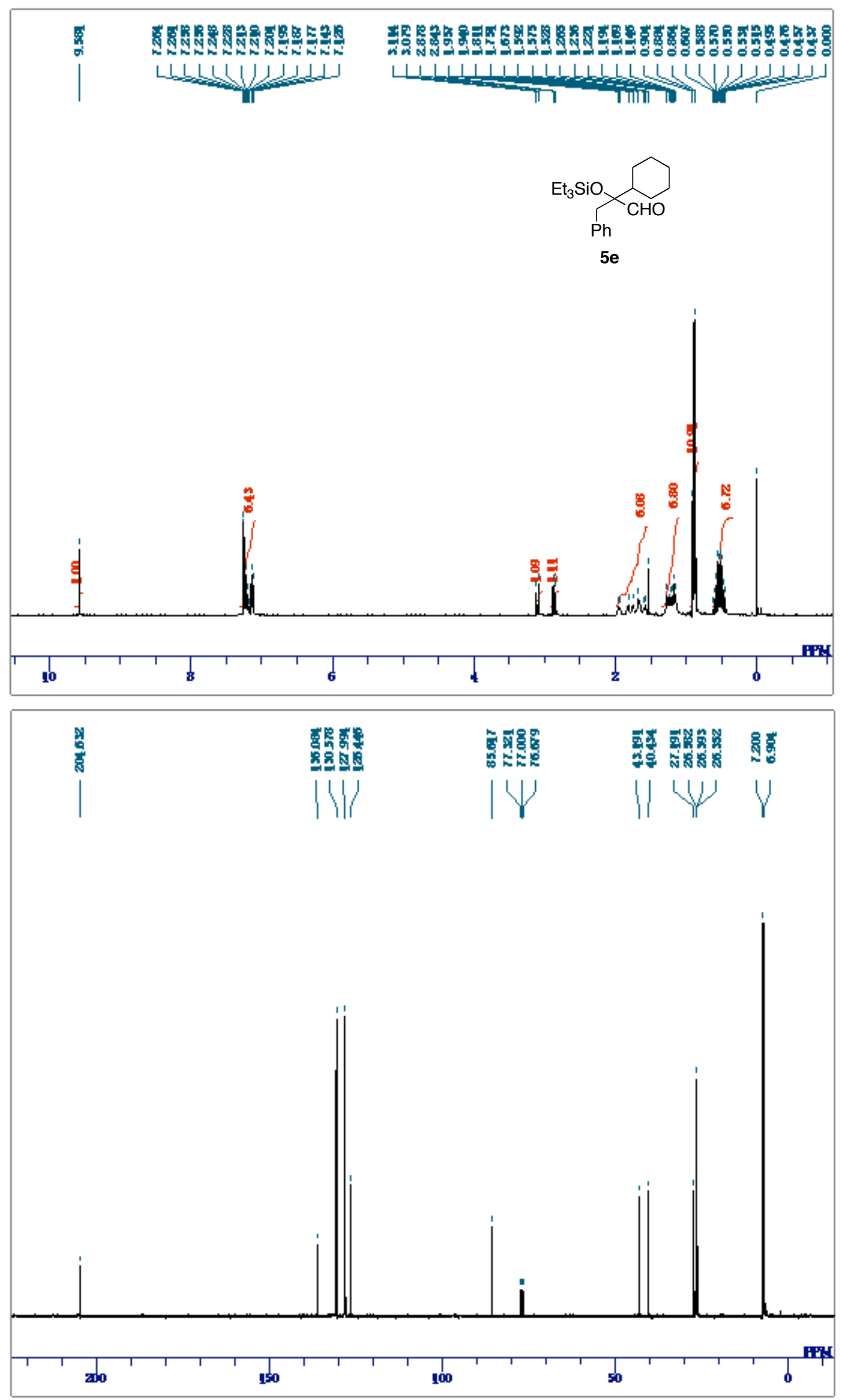



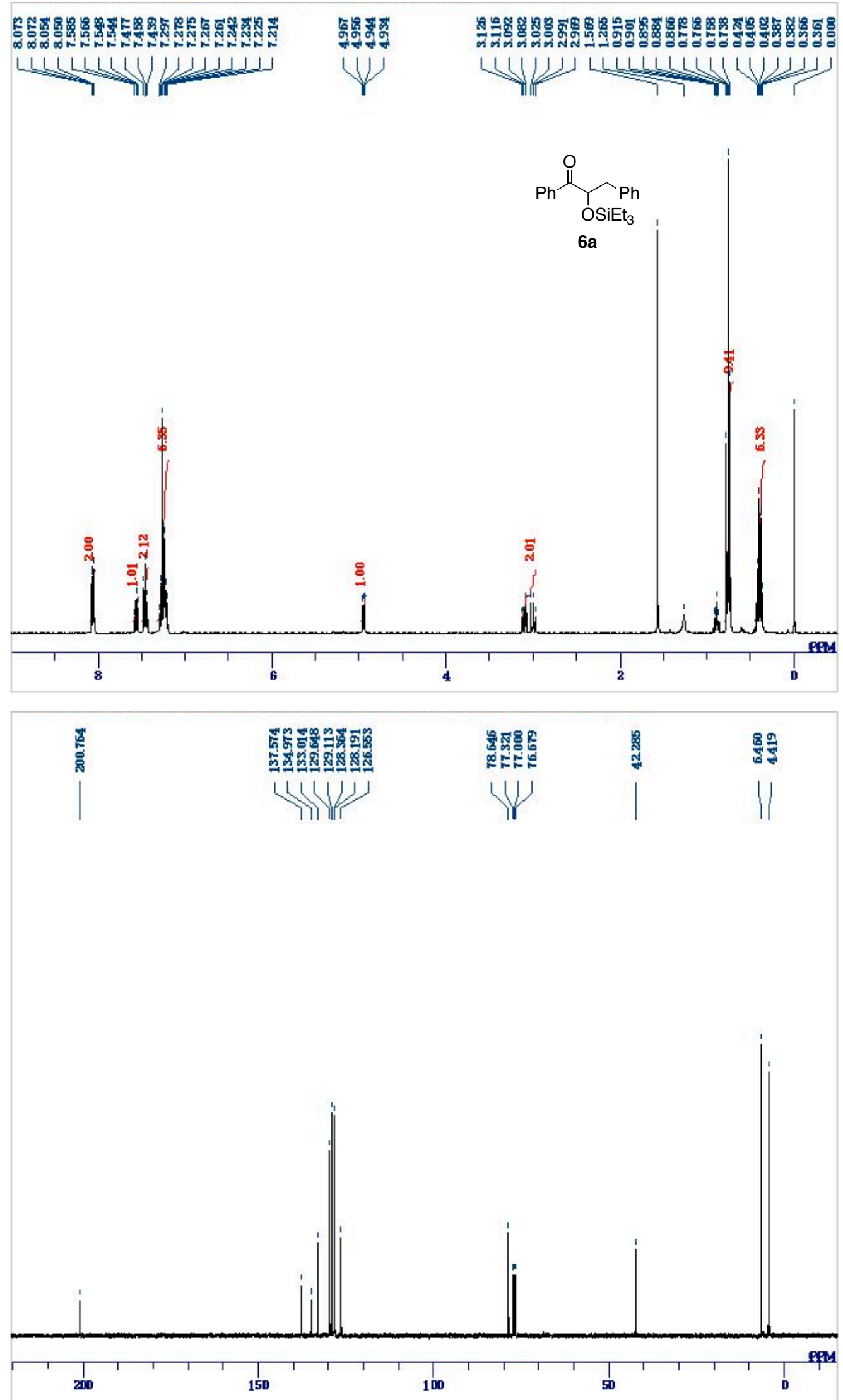

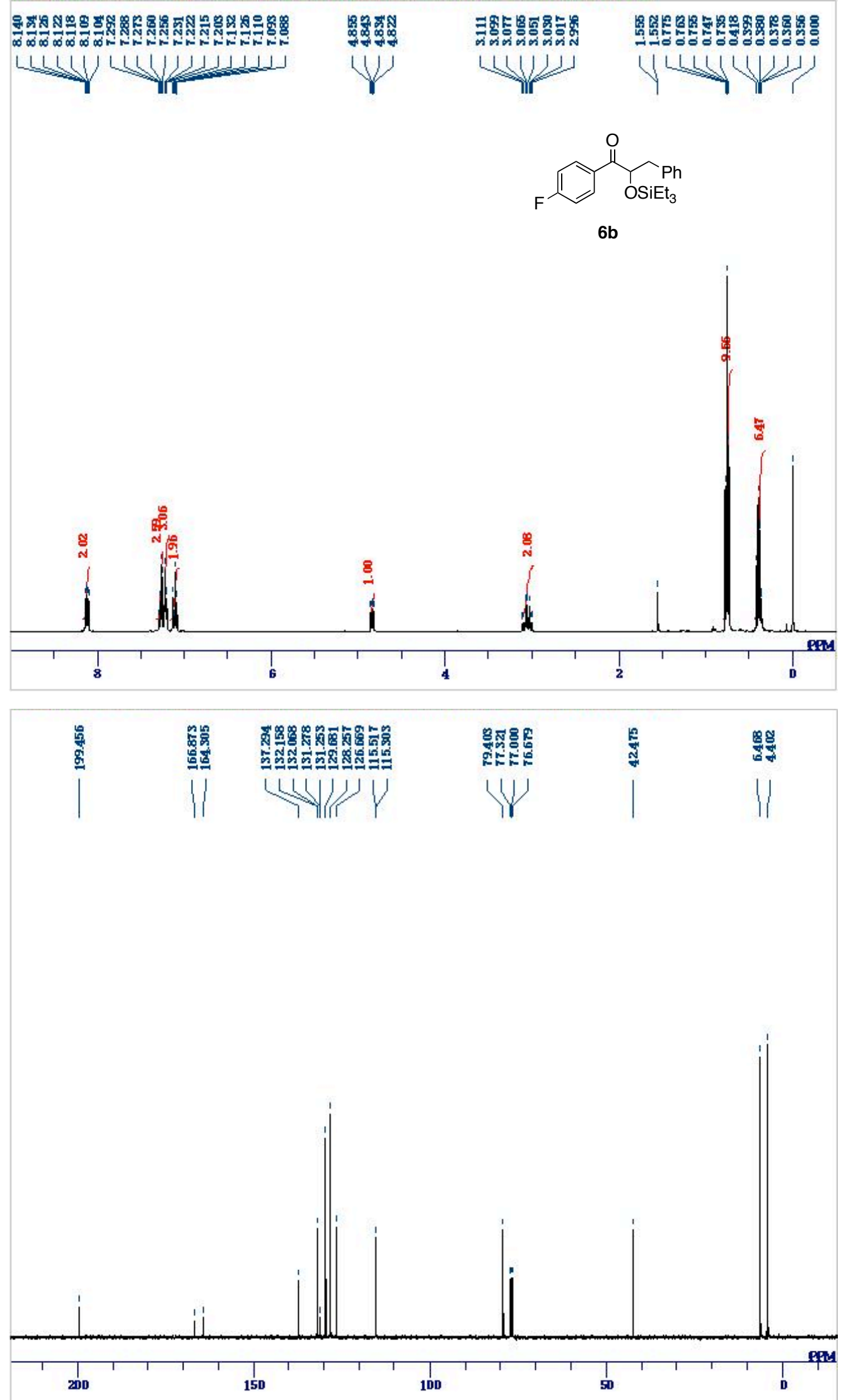


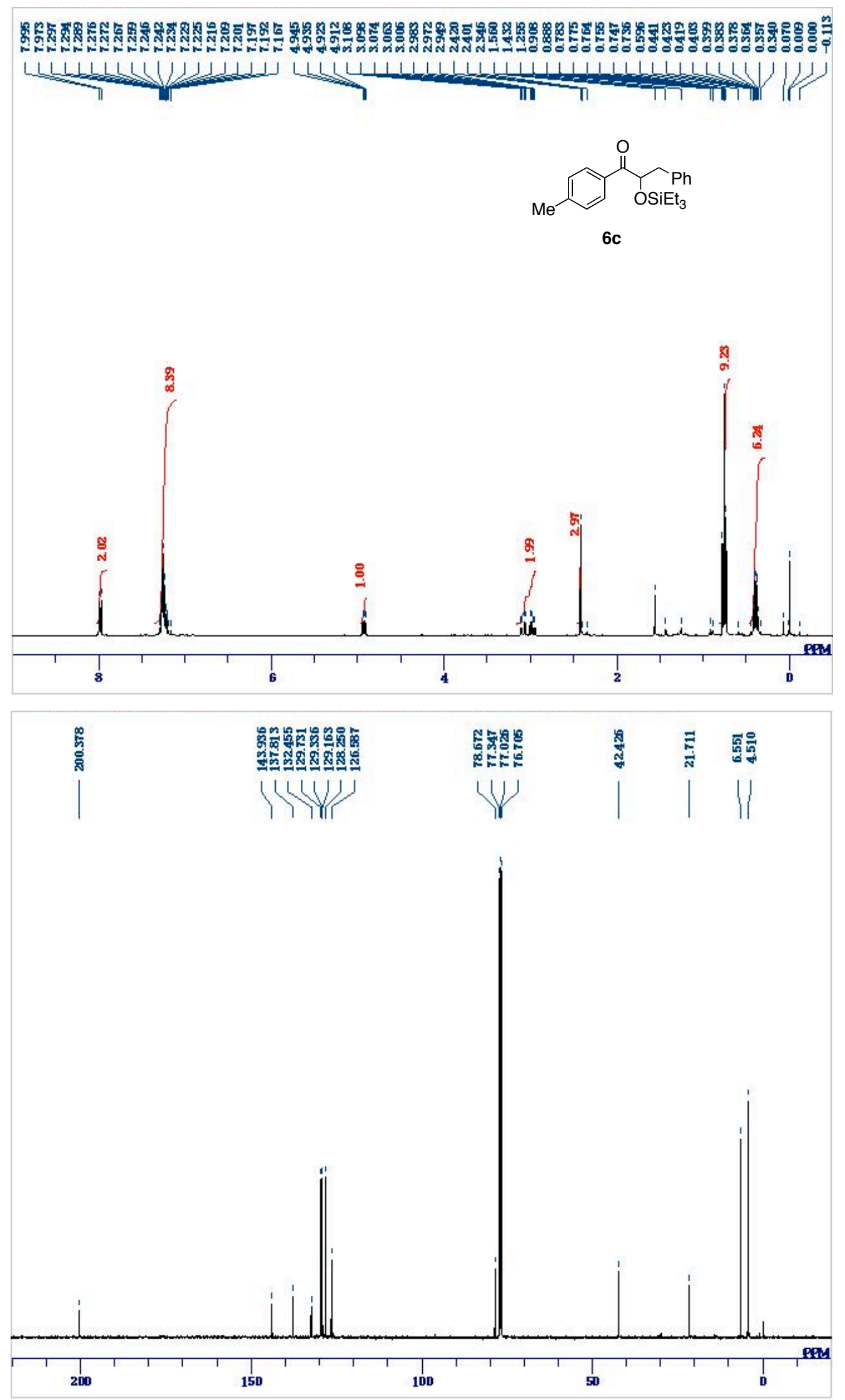




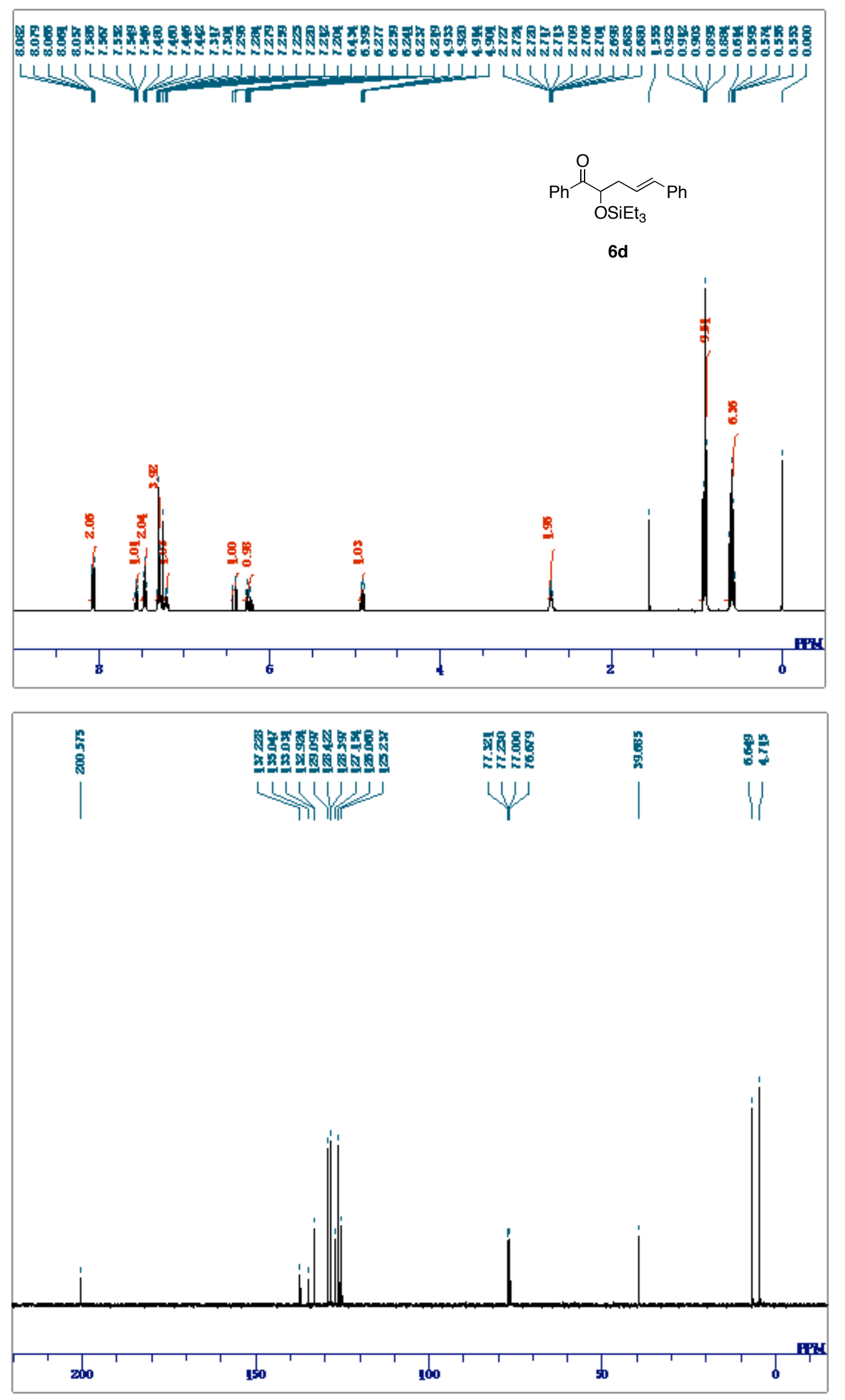



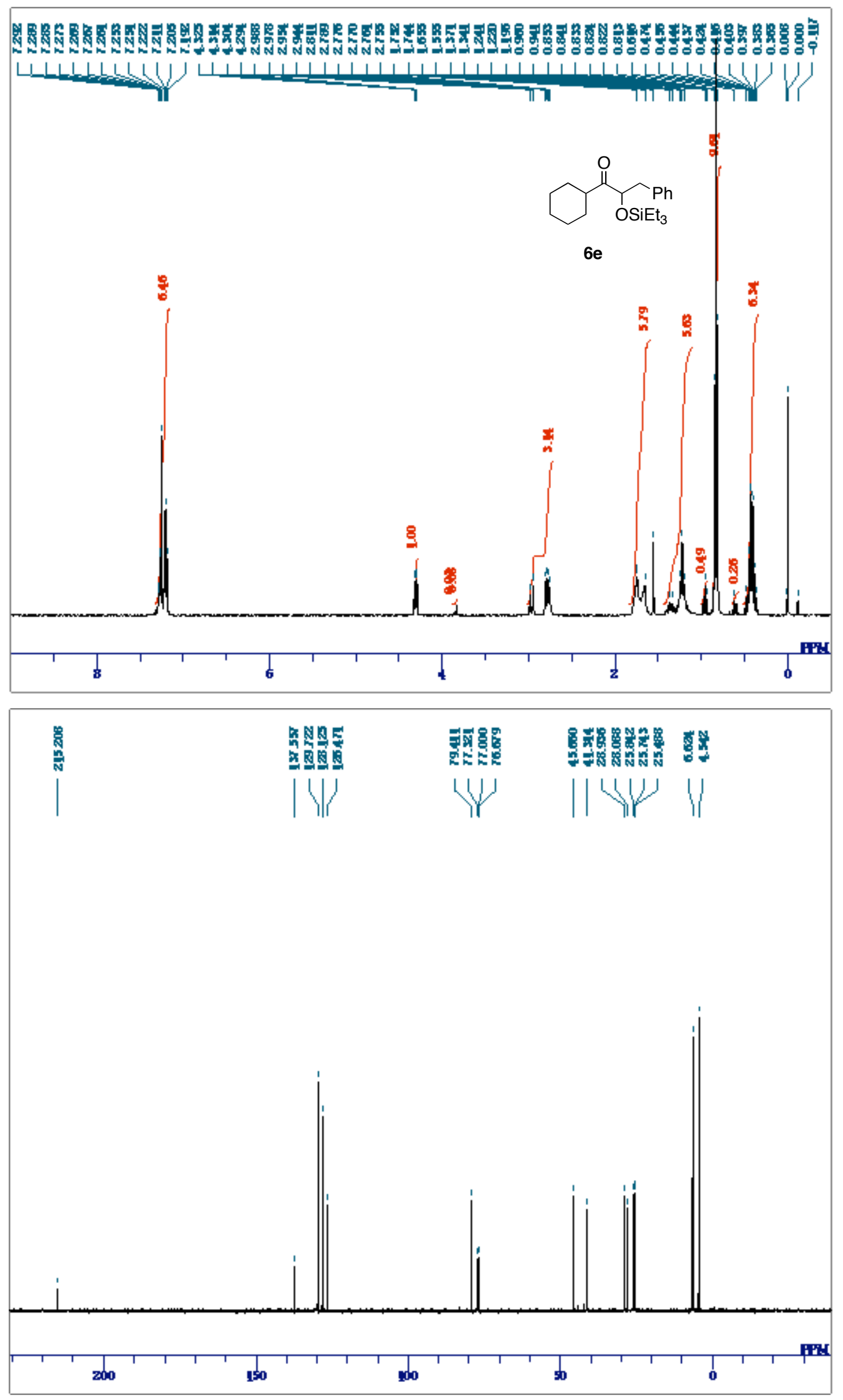